\title{
SUPPLEMENTARY DATA FOR
}

\section{Evaluation of Volatility and Thermal Stability in Monomeric and Dimeric Lanthanide(III) Complexes Containing Enaminolate Ligands}

\author{
Navoda Jayakodiarachchi, ${ }^{\dagger}$ Paul G. Evans, ${ }^{\ddagger}$ Cassandra L. Ward, ${ }^{\dagger}$ and Charles H. Winter*, \\ ${ }^{\dagger}$ Department of Chemistry, Wayne State University, Detroit, Michigan 48202 \\ ${ }^{\ddagger}$ Department of Materials Science and Engineering, University of Wisconsin-Madison, Madison, \\ Wisconsin 53706
}

X-Ray Crystallography Details:

Diffraction data for 1-3, 7-9, 13, and 14 were measured on a Bruker X8 APEX-II kappa geometry diffractometer with Mo radiation and a graphite monochromator. Using APEX3, frames were collected at $100 \mathrm{~K}$ with the APEX II CCD detector at $40 \mathrm{~mm}$ and $0.5^{\circ}$ between each frame. The diffraction intensities were integrated using SAINT. A multiscan absorption correction was applied with SADABS for 1-3, 8, and 14, and TWINABS for 7, 9, and 13. The crystal structures were solved using a dual-space approach as implemented in SHELXT [1] and difference Fourier $(\Delta \mathrm{F})$ maps during least-squares refinement, as embedded in SHELXL-2018 [2] running under Olex2 [3]. All non-hydrogen atoms were refined anisotropically. The hydrogen atoms were positioned with idealized geometry and refined isotropically using a riding model.

Additionally, 1 exhibited disorder over two sites between one $L^{1}$ ligand and one $L^{1} \mathrm{H}$ ligand with site occupancy factors (SOF) of 0.570 and 0.430 . Structure 7 was determined to be a two-component nonmerohedral twin related to each other by a $180^{\circ}$ rotation angle around the direct $\left[\begin{array}{ll}1 & 0\end{array}\right.$ 0] rotation vector. Refinement was performed using the HKLF-5 with reflections from the main domain, which led to a batch scale factor (BASF) parameter of $0.3953(12)$. One of the ligands was disordered between $\mathrm{K}^{2} \mathrm{~L}^{1}$ ligand and an oxygen-bound $\mathrm{K}^{1}-\mathrm{L}^{1}$ ligand and refined with SOF of 0.655 and 0.345 , respectively. Structure 9 exhibited whole-molecule disorder with SOF of 0.55 and 0.45 . The crystal was also a two-component nonmerohedral twin related to each other by a $180^{\circ}$ rotation angle around the reciprocal $\left[\begin{array}{llll}1 & 0 & 1\end{array}\right]$ rotation vector. Refinement was performed using the HKLF-5 with reflections from the main domain, which lead to a BASF parameter of $0.114(4)$. Structure 13 was a two-component non-merohedral twin related to each other by a $180^{\circ}$ rotation angle around the direct [-1 $\left.0-1\right]$ rotation vector. Refinement was performed using the HKLF-5 with reflections from the main domain, which lead to a batch scale factor (BASF) parameter of $0.0778(14)$. Two of the ligands were disordered over two sites and refined with SOF of 0.857 and 0.143. Structure 14 had a disordered piperidinyl substituent over two sites (0.582:0.418).

[1] Sheldrick, G.M., "SHELXT- Integrated space-group and crystal structure determination" (2015) Acta Cryst. A71, 3-8.

[2] Sheldrick, G.M., "Crystal structure refinement with SHELXL" (2015) Acta Cryst. C71, 3-8.

[3] Dolomanov, O.V., Bourhis, L.J., Gildea, R.J, Howard, J.A.K. \& Puschmann, H. (2009), “OLEX2: A complete structure solution refinement and analysis program", J. Appl. Cryst. 42, 339-341. 
Table S1. Crystal Data and Data Collection Parameters for 1-3 and 7-9.

\begin{tabular}{|c|c|c|c|c|c|c|}
\hline & 1 & 2 & 3 & 7 & 8 & 9 \\
\hline Formula & $\mathrm{C}_{32} \mathrm{H}_{65} \mathrm{~N}_{4} \mathrm{O}_{4} \mathrm{Pr}$ & $\mathrm{C}_{32} \mathrm{H}_{65} \mathrm{ErN}_{4} \mathrm{O}_{4}$ & $\mathrm{C}_{32} \mathrm{H}_{65} \mathrm{~N}_{4} \mathrm{O}_{4} \mathrm{Y}$ & $\mathrm{C}_{48} \mathrm{H}_{96} \mathrm{~N}_{6} \mathrm{Nd}_{2} \mathrm{O}_{6}$ & $\mathrm{C}_{24} \mathrm{H}_{48} \mathrm{LuN}_{3} \mathrm{O}_{3}$ & $\mathrm{C}_{60} \mathrm{H}_{108} \mathrm{La}_{2} \mathrm{~N}_{6} \mathrm{O}_{6}$ \\
\hline FW & 710.79 & 737.14 & 658.79 & 1141.78 & 601.62 & 1287.34 \\
\hline $\begin{array}{l}\text { space } \\
\text { group }\end{array}$ & $\mathrm{C} 2 / \mathrm{c}$ & $\mathrm{P} 2_{1} / \mathrm{n}$ & $\mathrm{P} 2_{1} / \mathrm{n}$ & $\mathrm{P} 2_{1} / \mathrm{n}$ & $\mathrm{P} 2_{1} / \mathrm{n}$ & $\mathrm{C} 2 / \mathrm{c}$ \\
\hline a $(\AA)$ & $30.5956(19)$ & $11.7598(4)$ & $11.7855(5)$ & $11.1563(12)$ & $9.7515(6)$ & $21.6485(9)$ \\
\hline$b(\AA)$ & $17.0132(8)$ & $16.0023(6)$ & $16.0308(6)$ & $12.9057(14)$ & $21.5850(14)$ & $16.9744(8)$ \\
\hline$c(\AA)$ & $15.9490(8)$ & $20.3810(7)$ & $20.3944(7)$ & $39.627(4)$ & $14.4493(10)$ & $17.2690(8)$ \\
\hline$\beta\left(^{\circ}\right)$ & $114.704(2)$ & $103.9960(10)$ & $104.1050(10)$ & $97.567(3)$ & $108.164(2)$ & $95.5350(10)$ \\
\hline$V\left(\AA^{3}\right)$ & $7542.1(7)$ & $3721.5(2)$ & $3737.0(2)$ & $5655.8(10)$ & $2889.8(3)$ & $6316.3(5)$ \\
\hline$Z$ & 8 & 4 & 4 & 4 & 4 & 4 \\
\hline Temp (K) & 100(1) & $100(1)$ & $100(1)$ & $100(1)$ & $100(1)$ & $100(1)$ \\
\hline $\begin{array}{l}\rho_{\text {calcd }}(\mathrm{g} \\
\left.\mathrm{cm}^{-3}\right)\end{array}$ & 1.252 & 1.316 & 1.171 & 1.341 & 1.383 & 1.1 .354 \\
\hline$\lambda(\AA ̊)$ & 0.71073 & 0.71073 & 0.71073 & 0.71073 & 0.71073 & 0.71073 \\
\hline$\mu\left(\mathrm{mm}^{-1}\right)$ & 1.328 & 2.291 & 1.597 & 1.862 & 3.441 & 1.385 \\
\hline$R(F)(\%)$ & 4.58 & 2.67 & 3.26 & 6.89 & 2.16 & 9.27 \\
\hline$w R(F)(\%)$ & 11.60 & 5.27 & 7.09 & 11.81 & 4.40 & 27.87 \\
\hline
\end{tabular}

$\mathrm{R}(\mathrm{F})=\Sigma\|\mathrm{Fo}|-| \mathrm{Fc}\| / \Sigma|\mathrm{Fo}| ; w R(\mathrm{~F})=\left[\sum w\left(\mathrm{Fo}^{2}-\mathrm{Fc}^{2}\right)^{2} / \sum w\left(\mathrm{Fo}^{2}\right)^{2}\right]^{1 / 2}$ for I $>2 \sigma(\mathrm{I})$. 
Table S2. Crystal Data and Data Collection Parameters for $\mathbf{1 3}$ and $\mathbf{1 4 .}$

\begin{tabular}{|c|c|c|}
\hline & 13 & 14 \\
\hline Formula & $\mathrm{C}_{33} \mathrm{H}_{60} \mathrm{ErN}_{3} \mathrm{O}_{3}$ & $\mathrm{C}_{33} \mathrm{H}_{60} \mathrm{LuN}_{3} \mathrm{O}_{3}$ \\
\hline FW & 714.10 & 721.81 \\
\hline $\begin{array}{l}\text { space } \\
\text { group }\end{array}$ & $\mathrm{P} 2_{1}$ & $\mathrm{P} 2_{1}$ \\
\hline a $(\AA ̊)$ & $9.8447(7)$ & $9.8745(5)$ \\
\hline$b(\AA)$ & $15.9493(11)$ & $15.8943(8)$ \\
\hline$c(\AA)$ & $11.6776(8)$ & 11.6918(6) \\
\hline$\beta\left(^{\circ}\right)$ & $109.372(2)$ & $109.266(2)$ \\
\hline$V\left(\AA^{3}\right)$ & $1729.8(2)$ & $1732.24(15)$ \\
\hline$Z$ & 2 & 2 \\
\hline Temp (K) & $100(1)$ & $100(1)$ \\
\hline $\begin{array}{l}\rho_{\text {calcd }}(\mathrm{g} \\
\left.\mathrm{cm}^{-3}\right)\end{array}$ & 1.371 & 1.384 \\
\hline$\lambda(\AA)$ & 0.71073 & 0.71073 \\
\hline$\mu\left(\mathrm{mm}^{-1}\right)$ & 2.460 & 2.883 \\
\hline$R(F)(\%)$ & 4.10 & 2.94 \\
\hline$w R(F)(\%)$ & 9.03 & 6.14 \\
\hline $\begin{array}{l}\text { Flack } \\
\text { Parameter }\end{array}$ & $-0.012(9)$ & $0.022(13)$ \\
\hline
\end{tabular}

$\mathrm{R}(\mathrm{F})=\Sigma\|\mathrm{Fo}|-| \mathrm{Fc}\| / \Sigma|\mathrm{Fo}| ; w R(\mathrm{~F})=\left[\sum w\left(\mathrm{Fo}^{2}-\mathrm{Fc}^{2}\right)^{2} / \Sigma w\left(\mathrm{Fo}^{2}\right)^{2}\right]^{1 / 2}$ for $\mathrm{I}>2 \sigma(\mathrm{I})$. 
Table S3. Bond lengths and angles for 9 .

\begin{tabular}{|c|c|c|c|c|c|}
\hline Atom & Atom & Length/Å & Atom & Atom & Length/̊̊ \\
\hline La1A & ${\operatorname{La} 1 A^{1}}^{1}$ & $4.1738(15)$ & C23A & $C 25 A$ & $1.515(6)$ \\
\hline La1A & $01 A^{1}$ & $2.569(12)$ & C23A & C24A & $1.517(6)$ \\
\hline La1A & 01A & $2.450(15)$ & La1B & La1B $^{1}$ & $4.1615(18)$ \\
\hline La1A & $\mathrm{O} 2 \mathrm{~A}$ & $2.319(8)$ & La1B & О3В & $2.326(8)$ \\
\hline La1A & $\mathrm{C} 2 \mathrm{~A}$ & $2.999(15)$ & La1B & N2B & $2.641(17)$ \\
\hline La1A & N1A & $2.733(12)$ & La1B & O1B & $2.42(2)$ \\
\hline La1A & C1A & $2.992(15)$ & La1B & $\mathrm{O}^{1} \mathrm{~B}^{1}$ & $2.54(2)$ \\
\hline La1A & O3A & $2.322(8)$ & La1B & C30B & $2.95(3)$ \\
\hline La1A & N3A & $2.891(15)$ & La1B & O2B & $2.313(8)$ \\
\hline O1A & $C 2 A^{1}$ & $1.35(2)$ & La1B & N1B & $2.743(17)$ \\
\hline $\mathrm{O} 2 \mathrm{~A}$ & $\mathrm{C} 12 \mathrm{~A}$ & $1.295(12)$ & La1B & $\mathrm{C} 2 \mathrm{~B}^{1}$ & $3.006(19)$ \\
\hline $\mathrm{C} 2 \mathrm{~A}$ & C1A & $1.33(2)$ & La1B & N3B & $3.043(17)$ \\
\hline $\mathrm{C} 2 \mathrm{~A}$ & C3A & $1.522(10)$ & La1B & $\mathrm{C}^{1 B^{1}}$ & $3.03(2)$ \\
\hline N1A & C1A & $1.416(13)$ & O3B & C22B & $1.306(12)$ \\
\hline$N 1 A$ & C10A & $1.488(10)$ & C27B & N2B & $1.469(10)$ \\
\hline N1A & C7A & $1.492(10)$ & C27B & $\mathrm{C} 28 \mathrm{~B}$ & $1.489(10)$ \\
\hline C13A & C15A & $1.517(6)$ & N2B & C21B & $1.422(13)$ \\
\hline C13A & C16A & $1.514(6)$ & N2B & C30B & $1.470(10)$ \\
\hline C13A & C12A & $1.525(10)$ & C22B & C21B & $1.308(15)$ \\
\hline $\mathrm{C} 13 \mathrm{~A}$ & C14A & $1.516(6)$ & C22B & C23B & $1.524(10)$ \\
\hline C3A & C5A & $1.516(6)$ & C12B & O2B & $1.286(12)$ \\
\hline C3A & C6A & $1.517(6)$ & C12B & C13B & $1.520(10)$ \\
\hline C3A & C4A & $1.515(6)$ & C12B & C11B & $1.279(15)$ \\
\hline C5B & C3B & $1.517(6)$ & C23B & C26B & $1.516(6)$ \\
\hline C27A & N3A & $1.497(10)$ & C23B & C24B & $1.516(6)$ \\
\hline C27A & C28A & $1.492(10)$ & C23B & C25B & $1.516(6)$ \\
\hline C30A & C29A & $1.493(10)$ & O1B & $\mathrm{C} 2 \mathrm{~B}$ & $1.33(3)$ \\
\hline
\end{tabular}




\begin{tabular}{|l|l|l|l|l|l|l|}
\hline C30A & N3A & $1.507(10)$ & & C29B & C30B & $1.491(10)$ \\
\hline C29A & C28A & $1.487(10)$ & & C29B & C28B & $1.487(10)$ \\
\hline C10A & C9A & $1.492(10)$ & & N1B & C10B & $1.486(10)$ \\
\hline C8A & C7A & $1.484(10)$ & & N1B & C7B & $1.487(10)$ \\
\hline C8A & C9A & $1.486(10)$ & & N1B & C1B & $1.44(3)$ \\
\hline N2A & C11A & $1.416(13)$ & & C9B & C10B & $1.487(10)$ \\
\hline N2A & C17A & $1.496(10)$ & & C9B & C8B & $1.487(10)$ \\
\hline N2A & C20A & $1.497(10)$ & & C19B & C18B & $1.497(10)$ \\
\hline C11A & C12A & $1.291(15)$ & & C19B & C20B & $1.481(10)$ \\
\hline C4B & C3B & $1.515(6)$ & & C3B & C2B & $1.523(10)$ \\
\hline C6B & C3B & $1.517(6)$ & & C7B & C8B & $1.490(10)$ \\
\hline C19A & C20A & $1.495(10)$ & & C2B & C1B & $1.40(3)$ \\
\hline C19A & C18A & $1.489(10)$ & & C13B & C15B & $1.515(6)$ \\
\hline C17A & C18A & $1.496(10)$ & & C13B & C16B & $1.517(6)$ \\
\hline O3A & C22A & $1.294(12)$ & & C13B & C14B & $1.519(6)$ \\
\hline C22A & C21A & $1.292(15)$ & & N3B & C11B & $1.412(13)$ \\
\hline C22A & C23A & $1.523(10)$ & & C17B & $1.487(10)$ \\
\hline C21A & N3A & $1.418(13)$ & & C20B & $1.477(10)$ \\
\hline C26A & C23A & $1.516(6)$ & & & & \\
\hline
\end{tabular}

${ }^{1} 1 / 2-X, 3 / 2-Y, 1-Z$

\begin{tabular}{|c|c|c|c|c|c|c|c|}
\hline Atom & Atom & Atom & Angle/ ${ }^{\circ}$ & Atom & Atom & Atom & Angle $^{\circ}$ \\
\hline 01A & La1A & $\operatorname{La}^{1} A^{1}$ & $34.7(3)$ & O3B & La1B & N2B & $61.7(4)$ \\
\hline $01 A^{1}$ & La1A & $\operatorname{La} A^{1}$ & $32.8(3)$ & O3B & La1B & 01B & $107.8(7)$ \\
\hline O1A & La1A & $01 A^{1}$ & $67.5(5)$ & O3B & La1B & C30B & $77.0(5)$ \\
\hline O1A & La1A & $\mathrm{C} 2 \mathrm{~A}$ & 93.0(4) & O3B & La1B & N1B & $134.7(6)$ \\
\hline $01 A^{1}$ & La1A & $\mathrm{C} 2 \mathrm{~A}$ & $26.6(5)$ & O3B & La1B & N3B & $78.9(5)$ \\
\hline $01 A^{1}$ & La1A & N1A & $62.6(4)$ & O3B & La1B & $\mathrm{C}_{1 \mathrm{~B}^{1}}$ & $106.6(6)$ \\
\hline O1A & La1A & N1A & $95.3(4)$ & N2B & La1B & C30B & $29.8(3)$ \\
\hline
\end{tabular}




\begin{tabular}{|c|c|c|c|c|c|c|c|}
\hline $01 A^{1}$ & La1A & C1A & $48.2(5)$ & $\mathrm{N} 2 \mathrm{~B}$ & La1B & N1B & $96.8(4)$ \\
\hline O1A & La1A & C1A & $105.2(4)$ & $\mathrm{N} 2 \mathrm{~B}$ & La1B & N3B & $81.0(5)$ \\
\hline O1A & La1A & N3A & 159.7(4) & $\mathrm{N} 2 \mathrm{~B}$ & La1B & $\mathrm{C}^{2} \mathrm{~B}^{1}$ & $84.9(5)$ \\
\hline $01 A^{1}$ & La1A & N3A & $126.1(4)$ & 01B & La1B & $\mathrm{N} 2 \mathrm{~B}$ & 167.9(7) \\
\hline $\mathrm{O} 2 \mathrm{~A}$ & La1A & $\operatorname{La} A^{1}$ & 103.1(4) & $\mathrm{O}^{1 \mathrm{~B}^{1}}$ & La1B & $\mathrm{N} 2 \mathrm{~B}$ & $117.9(6)$ \\
\hline $\mathrm{O} 2 \mathrm{~A}$ & La1A & $01 \mathrm{~A}$ & $111.7(5)$ & 01B & La1B & C30B & 159.2(7) \\
\hline $\mathrm{O} 2 \mathrm{~A}$ & La1A & $01 A^{1}$ & $90.9(4)$ & $\mathrm{O}^{1} \mathrm{~B}^{1}$ & La1B & C30B & $93.9(6)$ \\
\hline $\mathrm{O} 2 \mathrm{~A}$ & La1A & $\mathrm{C} 2 \mathrm{~A}$ & $88.2(4)$ & O1B & La1B & N1B & $95.0(8)$ \\
\hline $\mathrm{O} 2 \mathrm{~A}$ & La1A & N1A & $131.6(4)$ & O1B $^{1}$ & La1B & N1B & $62.7(7)$ \\
\hline $\mathrm{O} 2 \mathrm{~A}$ & La1A & C1A & $103.9(4)$ & O1B & La1B & N3B & $91.3(7)$ \\
\hline $\mathrm{O} 2 \mathrm{~A}$ & La1A & O3A & $133.4(5)$ & $\mathrm{O}^{1} \mathrm{~B}^{1}$ & La1B & N3B & $151.4(7)$ \\
\hline $\mathrm{O} 2 \mathrm{~A}$ & La1A & N3A & $84.8(5)$ & O1B & La1B & $\mathrm{C}^{1 \mathrm{~B}^{1}}$ & 104.7(8) \\
\hline $\mathrm{C} 2 \mathrm{~A}$ & La1A & $\operatorname{La}^{1} A^{1}$ & $58.7(3)$ & $\mathrm{O}^{1} \mathrm{~B}^{1}$ & La1B & $\mathrm{C}^{1 B^{1}}$ & $48.4(7)$ \\
\hline N1A & La1A & $\operatorname{La}^{1} A^{1}$ & $76.8(2)$ & C30B & La1B & N3B & 109.5(5) \\
\hline N1A & La1A & $\mathrm{C} 2 \mathrm{~A}$ & 49.6(4) & C30B & La1B & $\mathrm{C} \mathrm{B}^{1}$ & $55.2(5)$ \\
\hline N1A & La1A & C1A & $28.2(3)$ & O2B & La1B & O3B & $132.3(6)$ \\
\hline N1A & La1A & N3A & 81.1(4) & O2B & La1B & $\mathrm{N} 2 \mathrm{~B}$ & $87.1(6)$ \\
\hline C1A & La1A & $\operatorname{La}^{1} A^{1}$ & $75.2(3)$ & $\mathrm{O} 2 \mathrm{~B}$ & La1B & O1B & $97.2(8)$ \\
\hline C1A & La1A & $\mathrm{C} 2 \mathrm{~A}$ & 25.6(4) & $\mathrm{O} 2 \mathrm{~B}$ & La1B & C30B & $93.6(6)$ \\
\hline $\mathrm{O} A \mathrm{~A}$ & La1A & $\operatorname{La}^{1} A^{1}$ & 120.6(3) & O2B & La1B & N1B & $80.3(5)$ \\
\hline O3A & La1A & $01 A^{1}$ & $134.8(5)$ & O2B & La1B & N3B & $60.2(4)$ \\
\hline O3A & La1A & O1A & $97.4(4)$ & O2B & La1B & $\mathrm{C}^{1 B^{1}}$ & $105.4(5)$ \\
\hline O3A & La1A & C2A & $127.0(5)$ & N1B & La1B & C30B & $69.4(5)$ \\
\hline O3A & La1A & N1A & $77.6(4)$ & N1B & La1B & N3B & $140.5(4)$ \\
\hline O3A & La1A & C1A & $102.4(4)$ & N1B & La1B & $\mathrm{C}^{1 B^{1}}$ & $28.4(5)$ \\
\hline O3A & La1A & N3A & $62.3(4)$ & $\mathrm{C} 2 \mathrm{~B}^{1}$ & La1B & N3B & $167.9(4)$ \\
\hline N3A & La1A & $\operatorname{La}^{1} A^{1}$ & $156.0(3)$ & $C 2 B^{1}$ & La1B & $\mathrm{C}^{1 B^{1}}$ & $26.9(6)$ \\
\hline N3A & La1A & $\mathrm{C} 2 \mathrm{~A}$ & $99.5(4)$ & $\mathrm{C} \mathrm{B}^{1}$ & La1B & N3B & $160.2(5)$ \\
\hline N3A & La1A & C1A & $80.9(4)$ & C22B & O3B & La1B & $132.2(8)$ \\
\hline
\end{tabular}




\begin{tabular}{|c|c|c|c|c|c|c|c|}
\hline La1A & O1A & La1A $^{1}$ & $112.5(5)$ & $\mathrm{N} 2 \mathrm{~B}$ & C27B & C28B & $104.4(14)$ \\
\hline$C 2 A^{1}$ & O1A & La1A $^{1}$ & $94.8(10)$ & C27B & $\mathrm{N} 2 \mathrm{~B}$ & La1B & $112.5(11)$ \\
\hline$C 2 A^{1}$ & O1A & La1A & $147.8(11)$ & C27B & $\mathrm{N} 2 \mathrm{~B}$ & C30B & $111.5(9)$ \\
\hline C12A & $\mathrm{O} 2 \mathrm{~A}$ & La1A & $137.3(8)$ & C21B & $\mathrm{N} 2 \mathrm{~B}$ & La1B & $111.3(8)$ \\
\hline $01 A^{1}$ & $\mathrm{C} 2 \mathrm{~A}$ & La1A & $58.6(8)$ & C21B & $\mathrm{N} 2 \mathrm{~B}$ & C27B & $115.7(7)$ \\
\hline $01 A^{1}$ & $\mathrm{C} 2 \mathrm{~A}$ & C3A & $120.6(12)$ & C21B & $\mathrm{N} 2 \mathrm{~B}$ & C30B & $115.6(7)$ \\
\hline C1A & $\mathrm{C} 2 \mathrm{~A}$ & La1A & $76.9(9)$ & C30B & $\mathrm{N} 2 \mathrm{~B}$ & La1B & $86.9(12)$ \\
\hline $\mathrm{C} 1 \mathrm{~A}$ & $\mathrm{C} 2 \mathrm{~A}$ & $01 A^{1}$ & $118.7(12)$ & O3B & C22B & C21B & $113.0(9)$ \\
\hline C1A & $\mathrm{C} 2 \mathrm{~A}$ & C3A & 119.7(11) & O3B & C22B & C23B & $122.4(8)$ \\
\hline C3A & $\mathrm{C} 2 \mathrm{~A}$ & La1A & $128.1(7)$ & C21B & C22B & C23B & $123.6(9)$ \\
\hline $\mathrm{C} 1 \mathrm{~A}$ & N1A & La1A & $86.1(8)$ & O2B & C12B & C13B & 106.7(9) \\
\hline $\mathrm{C} 1 \mathrm{~A}$ & N1A & C10A & $114.8(7)$ & C11B & C12B & O2B & $118.4(11)$ \\
\hline $\mathrm{C} 1 \mathrm{~A}$ & N1A & C7A & $114.4(6)$ & C11B & C12B & C13B & $126.5(10)$ \\
\hline C10A & N1A & La1A & $114.7(8)$ & C22B & C21B & N2B & $121.5(11)$ \\
\hline C10A & N1A & C7A & $108.9(8)$ & C26B & C23B & C22B & $111.7(4)$ \\
\hline C7A & N1A & La1A & $116.6(8)$ & C26B & C23B & C24B & $107.1(5)$ \\
\hline C15A & C13A & C12A & $111.7(4)$ & C24B & C23B & C22B & $111.7(4)$ \\
\hline C16A & C13A & C15A & $107.2(5)$ & C25B & C23B & $\mathrm{C} 22 \mathrm{~B}$ & $111.8(4)$ \\
\hline C16A & C13A & C12A & $111.7(4)$ & C25B & C23B & C26B & $107.1(5)$ \\
\hline C16A & C13A & C14A & $107.3(5)$ & C25B & C23B & C24B & $107.2(5)$ \\
\hline C14A & C13A & C15A & $107.1(5)$ & $\mathrm{C} 2 \mathrm{~B}$ & O1B & La1B & $146.1(19)$ \\
\hline C14A & C13A & C12A & $111.5(4)$ & C28B & C29B & C30B & $110(2)$ \\
\hline $\mathrm{C} 2 \mathrm{~A}$ & C1A & La1A & $77.5(10)$ & N2B & C30B & La1B & $63.3(10)$ \\
\hline $\mathrm{C} 2 \mathrm{~A}$ & C1A & N1A & $123.3(11)$ & $\mathrm{N} 2 \mathrm{~B}$ & C30B & C29B & $100.5(14)$ \\
\hline N1A & C1A & La1A & $65.7(7)$ & C29B & C30B & La1B & $116.9(18)$ \\
\hline C5A & C3A & $\mathrm{C} 2 \mathrm{~A}$ & $111.8(4)$ & C12B & O2B & La1B & $132.4(9)$ \\
\hline C5A & C3A & C6A & $106.9(4)$ & C10B & N1B & La1B & $115.8(12)$ \\
\hline $\mathrm{C6A}$ & C3A & $\mathrm{C} 2 \mathrm{~A}$ & $111.7(4)$ & C10B & N1B & C7B & $109.4(9)$ \\
\hline C4A & C3A & $\mathrm{C} 2 \mathrm{~A}$ & $111.7(4)$ & C7B & N1B & La1B & $115.9(13)$ \\
\hline
\end{tabular}




\begin{tabular}{|c|c|c|c|c|c|c|c|}
\hline C4A & C3A & C5A & $107.3(5)$ & $\mathrm{C} \mathrm{B}^{1}$ & N1B & La1B & $86.7(11)$ \\
\hline C4A & C3A & C6A & $107.2(5)$ & $\mathrm{C} \mathrm{B}^{1}$ & N1B & C10B & $115.4(16)$ \\
\hline C28A & C27A & N3A & $99.9(13)$ & $\mathrm{C} \mathrm{B}^{1}$ & N1B & C7B & $112.1(16)$ \\
\hline C29A & C30A & N3A & $105.9(13)$ & C10B & C9B & C8B & $103.0(18)$ \\
\hline C28A & C29A & C30A & $104.3(18)$ & N1B & C10B & C9B & $108.8(13)$ \\
\hline N1A & C10A & C9A & $108.4(11)$ & C20B & C19B & C18B & $113(2)$ \\
\hline C7A & C8A & C9A & 111.7(14) & C5B & C3B & C6B & $107.0(5)$ \\
\hline $\mathrm{C} 8 \mathrm{~A}$ & C7A & $\mathrm{N} 1 \mathrm{~A}$ & $100.4(10)$ & C5B & C3B & $\mathrm{C} 2 \mathrm{~B}$ & $111.7(4)$ \\
\hline C11A & $\mathrm{N} 2 \mathrm{~A}$ & C17A & $114.1(7)$ & C4B & C3B & C5B & $107.3(5)$ \\
\hline C11A & $\mathrm{N} 2 \mathrm{~A}$ & C20A & 114.1(7) & C4B & C3B & C6B & $107.2(5)$ \\
\hline C17A & $\mathrm{N} 2 \mathrm{~A}$ & C20A & $108.2(9)$ & C4B & C3B & C2B & $111.7(4)$ \\
\hline C12A & C11A & $\mathrm{N} 2 \mathrm{~A}$ & $124.7(12)$ & C6B & C3B & C2B & $111.7(4)$ \\
\hline $\mathrm{O} 2 \mathrm{~A}$ & C12A & C13A & $117.8(8)$ & N1B & C7B & C8B & $101.3(13)$ \\
\hline C11A & C12A & $\mathrm{O} 2 \mathrm{~A}$ & $116.1(10)$ & C9B & C8B & C7B & $113.2(18)$ \\
\hline C11A & C12A & C13A & 124.6(9) & O1B & $\mathrm{C} 2 \mathrm{~B}$ & C3B & $122.5(17)$ \\
\hline $\mathrm{C} 8 \mathrm{~A}$ & C9A & C10A & $98.6(14)$ & O1B & $\mathrm{C} 2 \mathrm{~B}$ & C1B & 116.7(16) \\
\hline C18A & C19A & C20A & $112.2(17)$ & C1B & $\mathrm{C} 2 \mathrm{~B}$ & C3B & $119.4(13)$ \\
\hline C18A & C17A & $\mathrm{N} 2 \mathrm{~A}$ & $109.7(12)$ & C15B & C13B & C12B & $112.0(4)$ \\
\hline C19A & C20A & $\mathrm{N} 2 \mathrm{~A}$ & $99.5(12)$ & C15B & C13B & C16B & 107.2(5) \\
\hline C22A & O3A & La1A & $128.1(8)$ & C15B & C13B & C14B & $107.0(5)$ \\
\hline O3A & C22A & C23A & $113.4(8)$ & C16B & C13B & C12B & $111.8(4)$ \\
\hline C21A & C22A & O3A & $115.9(10)$ & C16B & C13B & C14B & $107.0(5)$ \\
\hline C21A & C22A & C23A & 124.7(9) & C14B & C13B & C12B & $111.6(4)$ \\
\hline C22A & C21A & N3A & $124.1(12)$ & C29A & C28A & C27A & 111.7(19) \\
\hline C26A & C23A & C22A & $111.8(4)$ & C19A & C18A & C17A & $97.2(15)$ \\
\hline C26A & C23A & C24A & $107.1(5)$ & C11B & N3B & La1B & $99.0(9)$ \\
\hline C25A & C23A & C22A & $111.8(4)$ & C11B & N3B & C17B & $114.9(7)$ \\
\hline C25A & C23A & C26A & $107.3(5)$ & C11B & N3B & C20B & $115.7(7)$ \\
\hline C25A & C23A & C24A & $107.0(5)$ & C17B & N3B & La1B & $115.4(12)$ \\
\hline
\end{tabular}




\begin{tabular}{|l|l|l|l|l|l|l|l|l|}
\hline C24A & C23A & C22A & $111.5(4)$ & & C20B & N3B & La1B & $100.6(12)$ \\
\hline C27A & N3A & La1A & $107.5(10)$ & & C20B & N3B & C17B & $110.0(9)$ \\
\hline C27A & N3A & C30A & $107.5(9)$ & & C12B & C11B & N3B & $125.9(13)$ \\
\hline C30A & N3A & La1A & $113.5(10)$ & & N3B & C17B & C18B & $104.0(15)$ \\
\hline C21A & N3A & La1A & $101.2(8)$ & & C17B & C18B & C19B & $98(2)$ \\
\hline C21A & N3A & C27A & $113.7(7)$ & & N3B & C20B & C19B & $88.1(16)$ \\
\hline C21A & N3A & C30A & $113.3(7)$ & & C29B & C28B & C27B & $100.2(19)$ \\
\hline & & & & & & & & \\
\hline
\end{tabular}


Table S4. Onset of volatilization and residual mass at $500{ }^{\circ} \mathrm{C}$ in the thermogravimetric analyses of 1-15.

\begin{tabular}{|c|c|c|}
\hline Compound & $\begin{array}{c}\text { Onset of } \\
\text { volatilization }\left({ }^{\circ} \mathrm{C}\right)\end{array}$ & Residual mass \% \\
\hline $\mathbf{1 ,} \operatorname{Pr}\left(\mathrm{L}^{1}\right)_{3}\left(\mathrm{~L}_{1} \mathrm{H}\right)$ & 96 & 15 \\
\hline $\mathbf{2 ,} \mathrm{Er}\left(\mathrm{L}^{1}\right)_{3}\left(\mathrm{~L}_{1} \mathrm{H}\right)$ & 80 & 19 \\
\hline $\mathbf{3 ,} \mathrm{Y}\left(\mathrm{L}^{1}\right)_{3}\left(\mathrm{~L}_{1} \mathrm{H}\right)$ & 92 & 7 \\
\hline $\mathbf{4 ,}\left[\operatorname{Pr}\left(\mathrm{L}^{1}\right)_{3}\right]_{n}$ & 176 & 5 \\
\hline $\mathbf{5 ,}\left[\mathrm{Er}\left(\mathrm{L}^{1}\right)_{3}\right]_{n}$ & 160 & 14 \\
\hline $\mathbf{6 ,}\left[\mathrm{Y}\left(\mathrm{L}^{1}\right)_{3}\right]_{n}$ & 158 & 10 \\
\hline $\mathbf{7 ,}\left[\mathrm{Nd}\left(\mathrm{L}^{1}\right)_{3}\right]_{2}$ & 153 & 20 \\
\hline $\mathbf{8 , ~} \mathrm{Lu}\left(\mathrm{L}^{1}\right)_{3}$ & 158 & 24 \\
\hline $\mathbf{9 ,}\left[\mathrm{La}\left(\mathrm{L}^{2}\right)_{3}\right]_{2}$ & 186 & 24 \\
\hline $\mathbf{1 0}, \mathrm{La}\left(\mathrm{L}^{3}\right)_{3}$ & 220 & 9 \\
\hline $\mathbf{1 1}, \operatorname{Pr}\left(\mathrm{L}^{3}\right)_{3}$ & 225 & 10 \\
\hline $\mathbf{1 2}, \mathrm{Nd}\left(\mathrm{L}^{3}\right)_{3}$ & 215 & 14 \\
\hline $\mathbf{1 3}, \mathrm{Er}\left(\mathrm{L}^{3}\right)_{3}$ & 230 & 12 \\
\hline $\mathbf{1 4}, \mathrm{Lu}\left(\mathrm{L}^{3}\right)_{3}$ & 220 & 13 \\
\hline $\mathbf{1 5}, \mathrm{Y}\left(\mathrm{L}^{3}\right)_{3}$ & 225 & 9 \\
\hline
\end{tabular}


Figure S1. ${ }^{1} \mathrm{H}$ NMR Spectrum of 3 in $\mathrm{C}_{6} \mathrm{D}_{6}$ at $23{ }^{\circ} \mathrm{C}$.
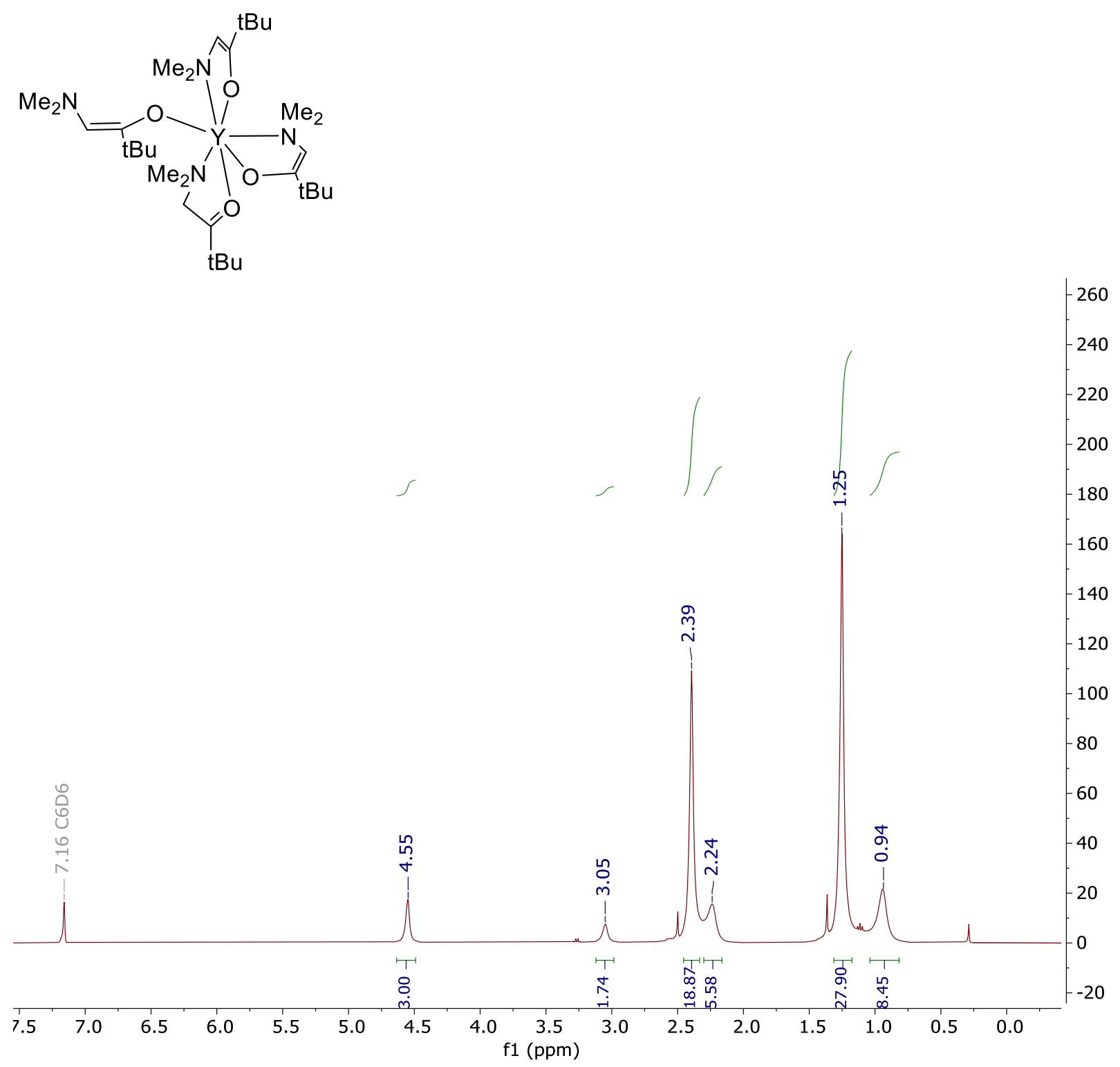
Figure S2. ${ }^{13} \mathrm{C}$ NMR Spectrum of 3 in $\mathrm{C}_{6} \mathrm{D}_{6}$ at $23^{\circ} \mathrm{C}$.
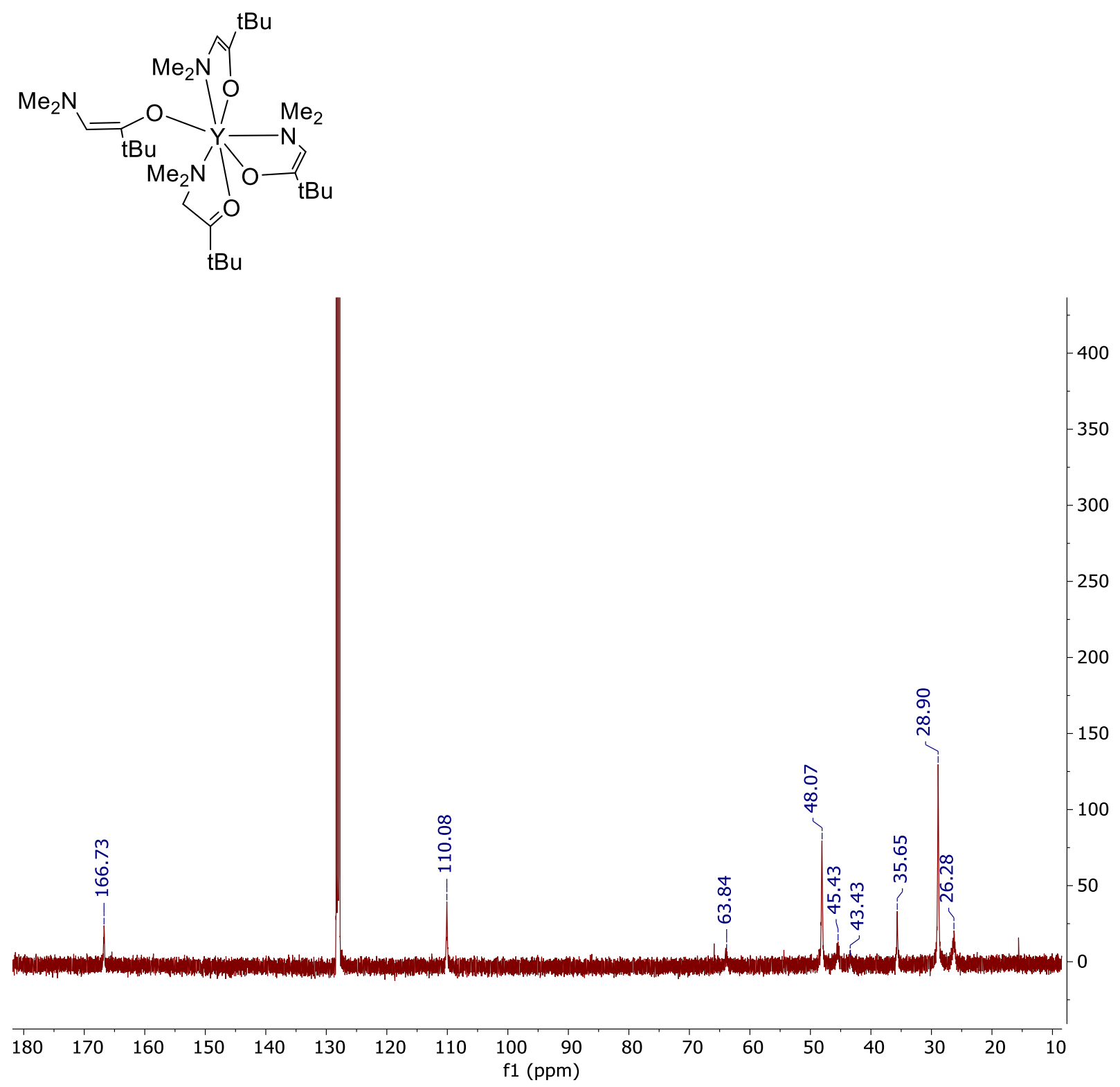
Figure S3. ${ }^{1} \mathrm{H}$ NMR Spectrum of 6 in $\mathrm{C}_{6} \mathrm{D}_{6}$ at $23{ }^{\circ} \mathrm{C}$.
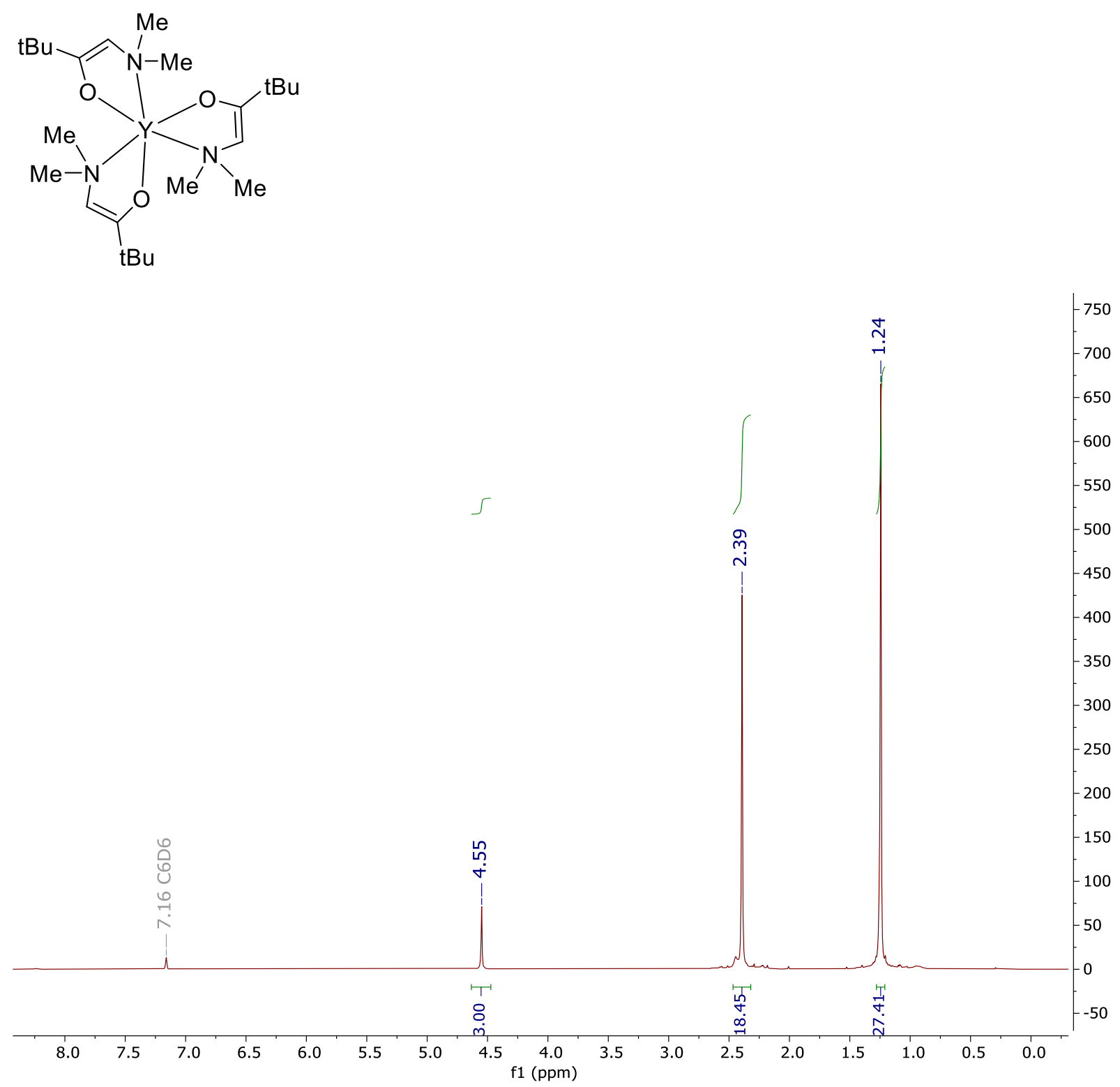
Figure S4. ${ }^{13} \mathrm{C}$ NMR Spectrum of 6 in $\mathrm{C}_{6} \mathrm{D}_{6}$ at $23^{\circ} \mathrm{C}$.

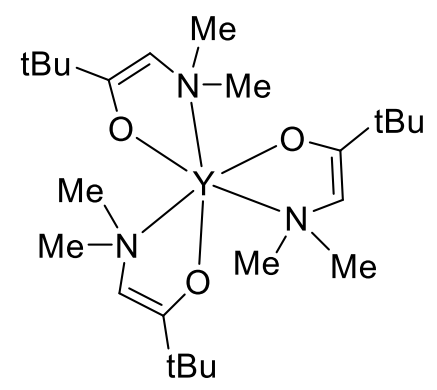

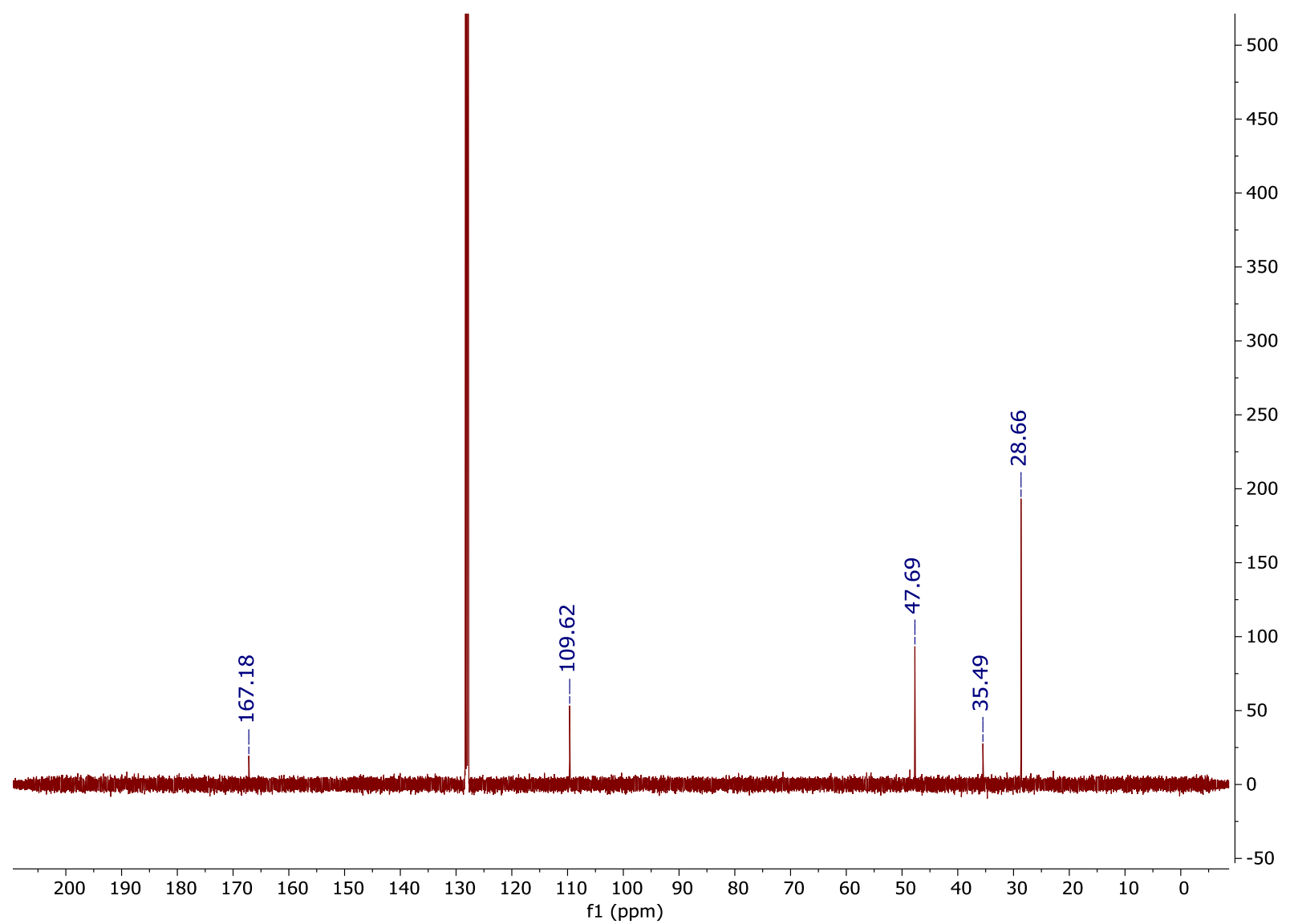


Figure S5. ${ }^{1} \mathrm{H}$ NMR Spectrum of 8 in $\mathrm{C}_{6} \mathrm{D}_{6}$ at $23{ }^{\circ} \mathrm{C}$.
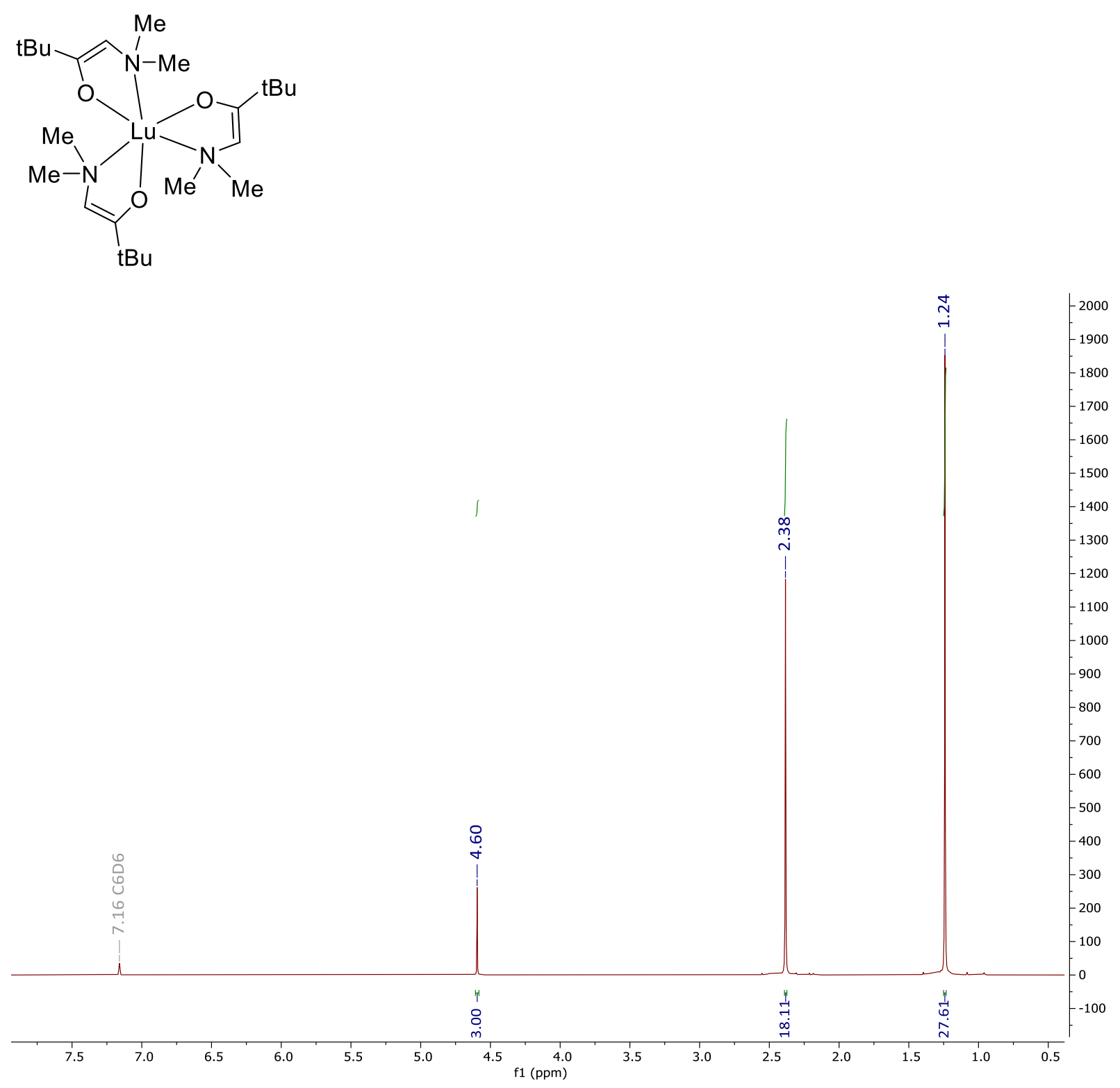
Figure S6. ${ }^{13} \mathrm{C}$ NMR Spectrum of 8 in $\mathrm{C}_{6} \mathrm{D}_{6}$ at $23^{\circ} \mathrm{C}$.

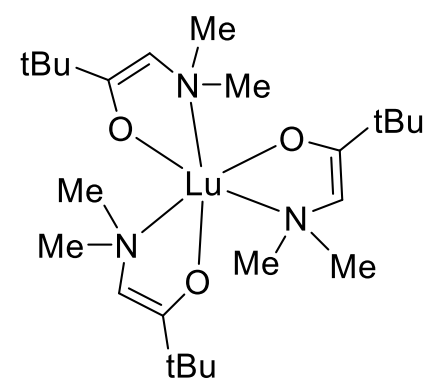

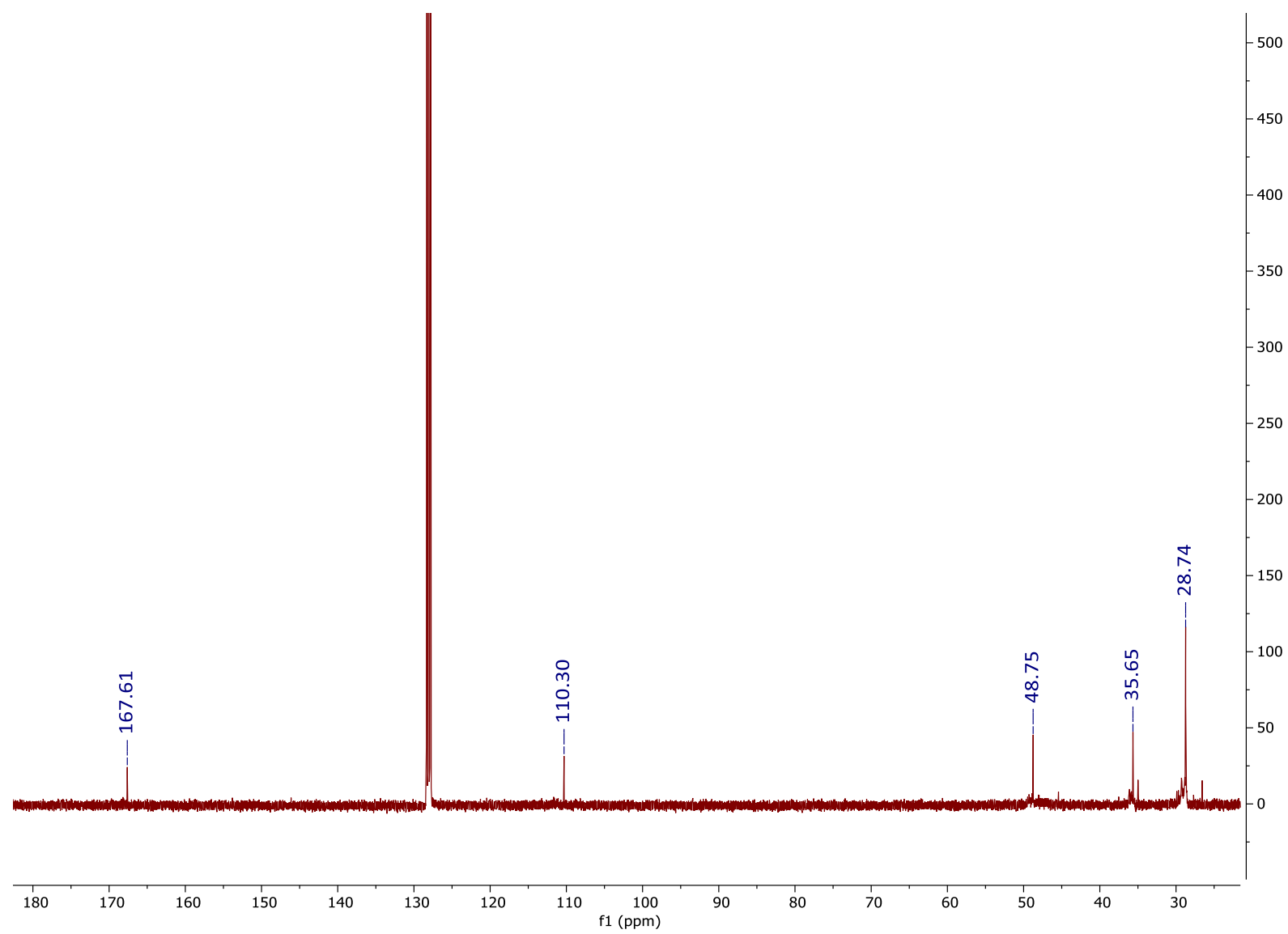


Figure S7. ${ }^{1} \mathrm{H}$ NMR Spectrum of 9 in $\mathrm{C}_{6} \mathrm{D}_{6}$ at $23{ }^{\circ} \mathrm{C}$.
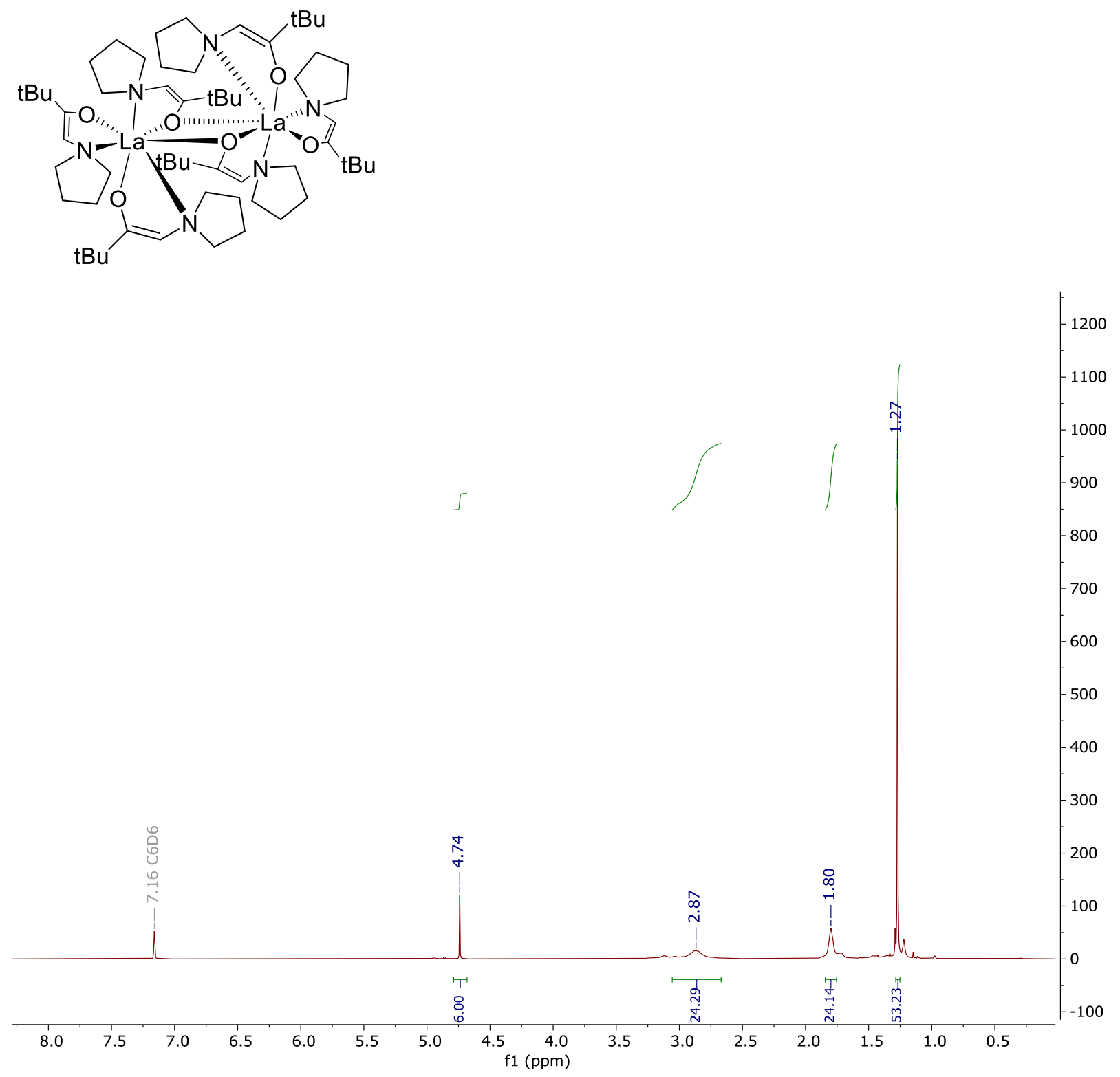
Figure S8. ${ }^{13} \mathrm{C}$ NMR Spectrum of 9 in $\mathrm{C}_{6} \mathrm{D}_{6}$ at $23^{\circ} \mathrm{C}$.

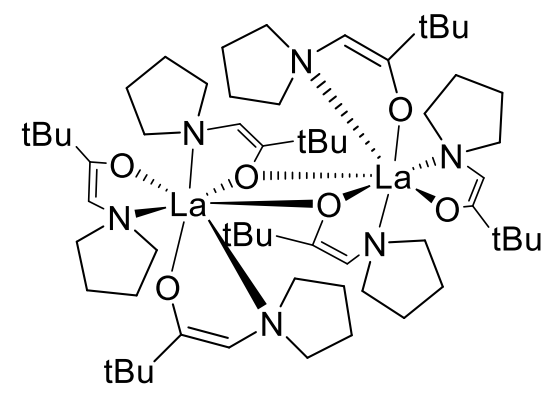

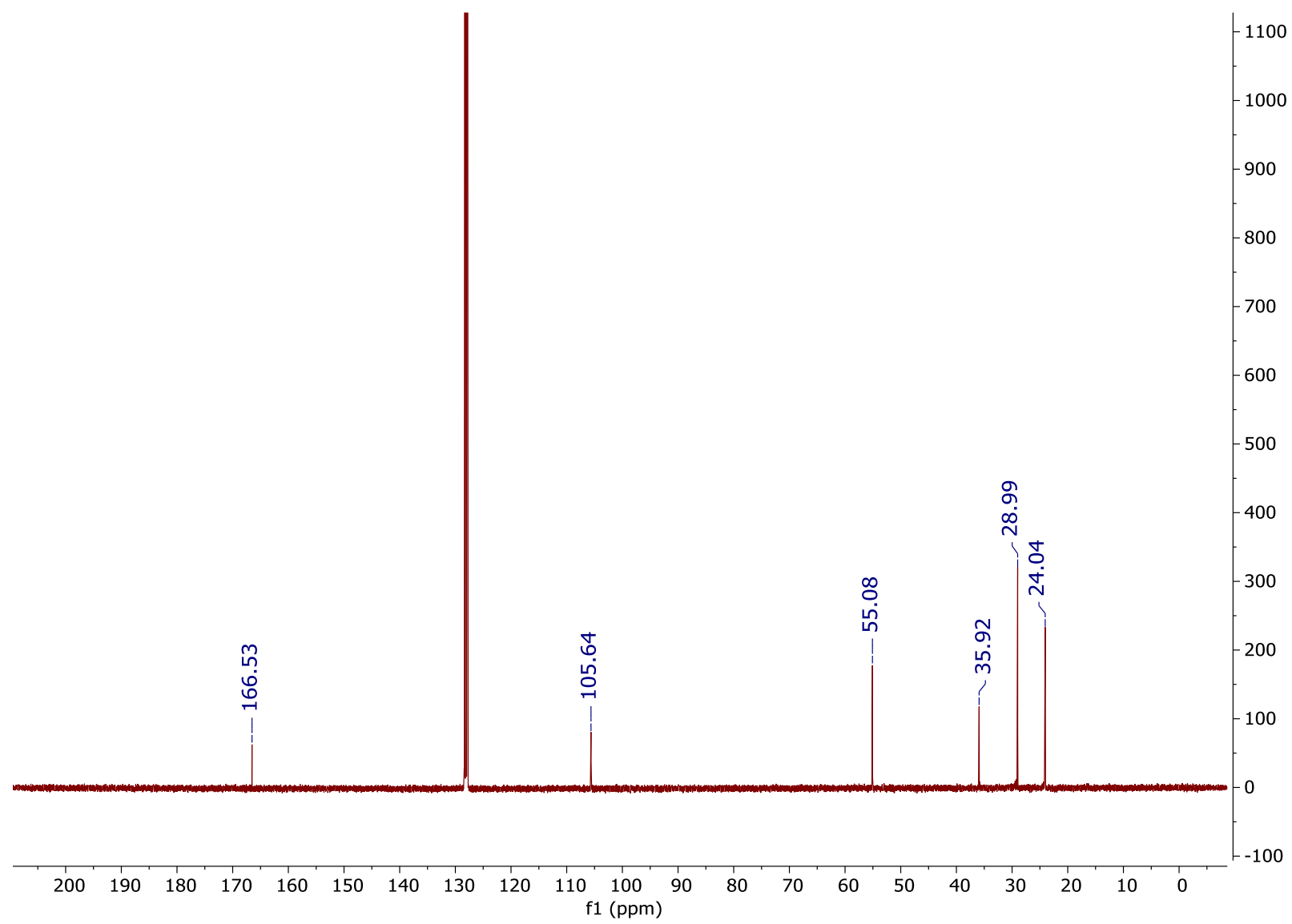


Figure S9. ${ }^{1} \mathrm{H}$ NMR Spectrum of 10 in $\mathrm{C}_{6} \mathrm{D}_{6}$ at $23^{\circ} \mathrm{C}$.

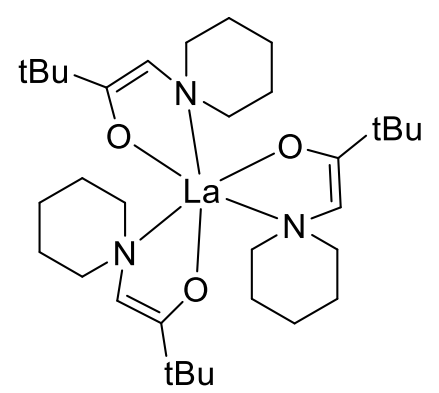

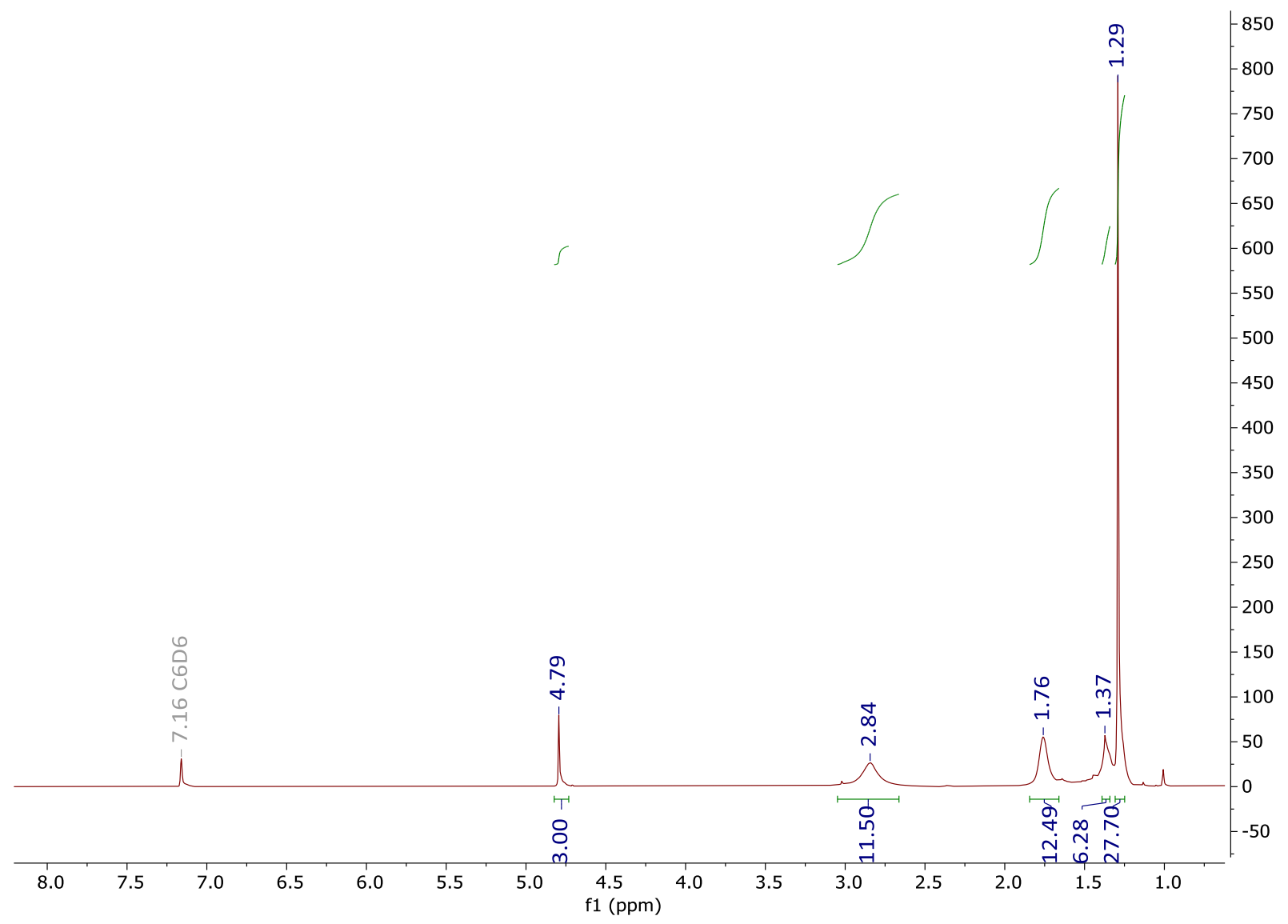


Figure S10. ${ }^{13} \mathrm{C}$ NMR Spectrum of 10 in $\mathrm{C}_{6} \mathrm{D}_{6}$ at $23{ }^{\circ} \mathrm{C}$.

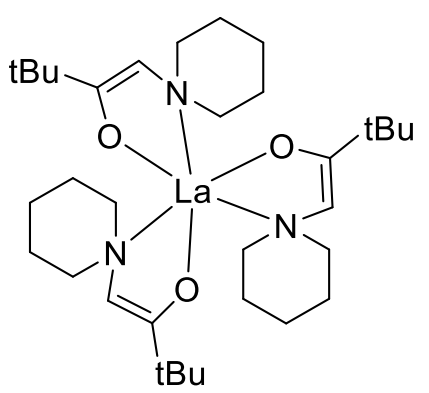

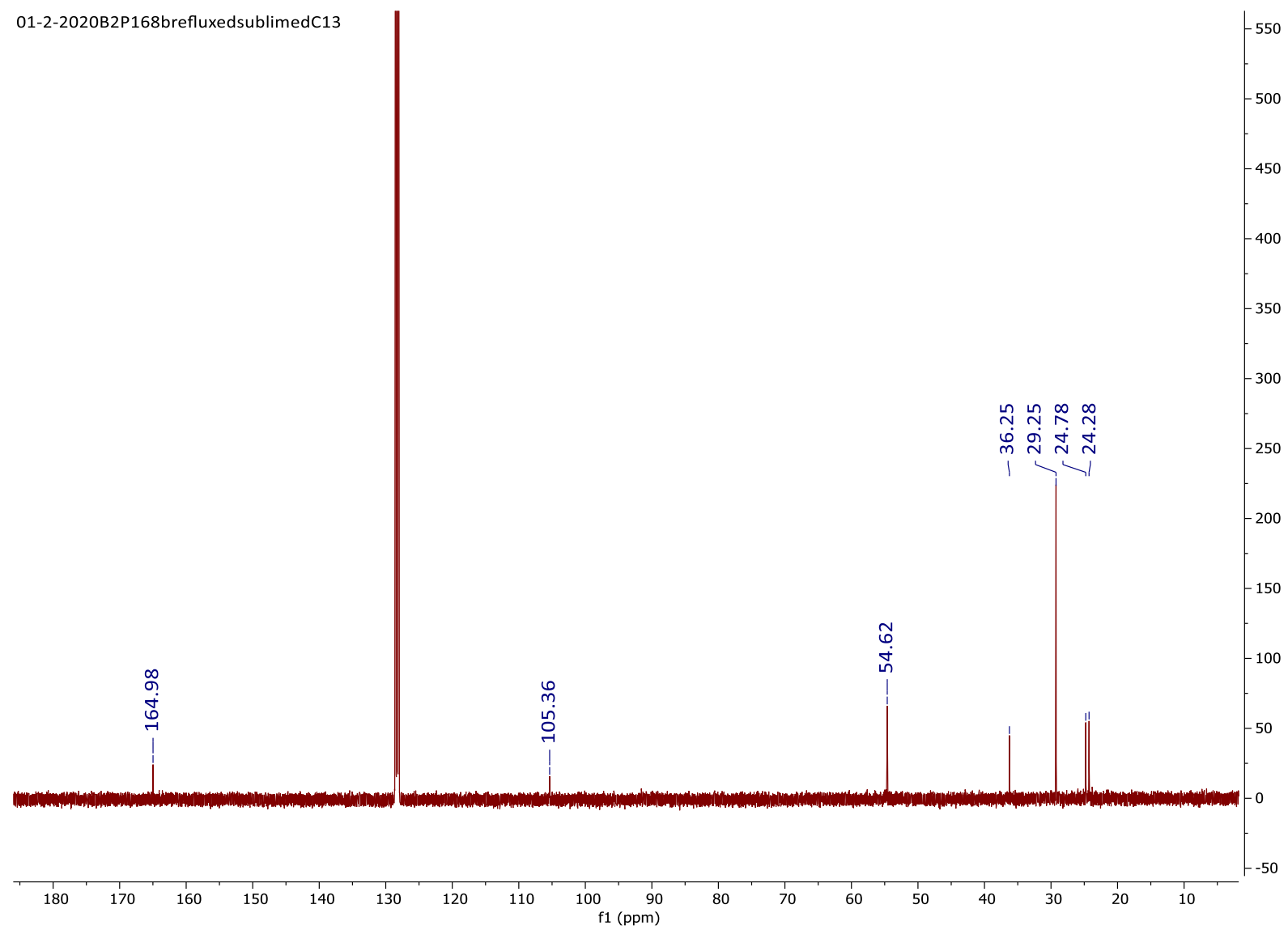


Figure S11. ${ }^{1} \mathrm{H}$ NMR Spectrum of 14 in $\mathrm{C}_{6} \mathrm{D}_{6}$ at $23{ }^{\circ} \mathrm{C}$.<smiles>CC(C)CC1=CN(C2(N3CCCCC3)C=C(CC(C)C)OC2(OC(C)CC(C)C)N2CCCCC2)CCCC1</smiles>

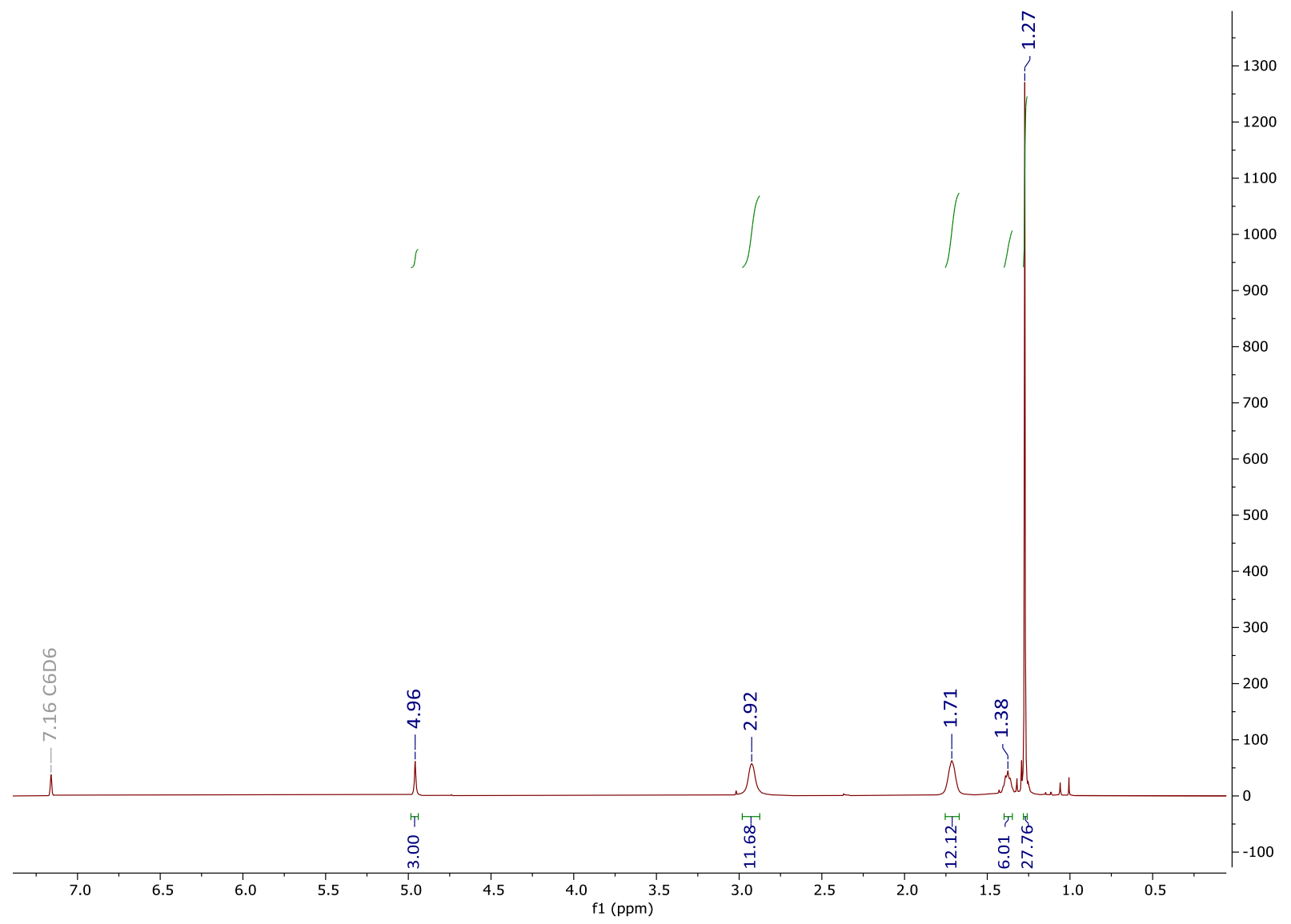


Figure S12. ${ }^{13} \mathrm{C}$ NMR Spectrum of 14 in $\mathrm{C}_{6} \mathrm{D}_{6}$ at $23{ }^{\circ} \mathrm{C}$.

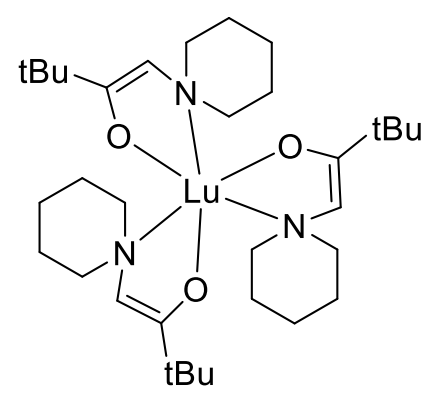

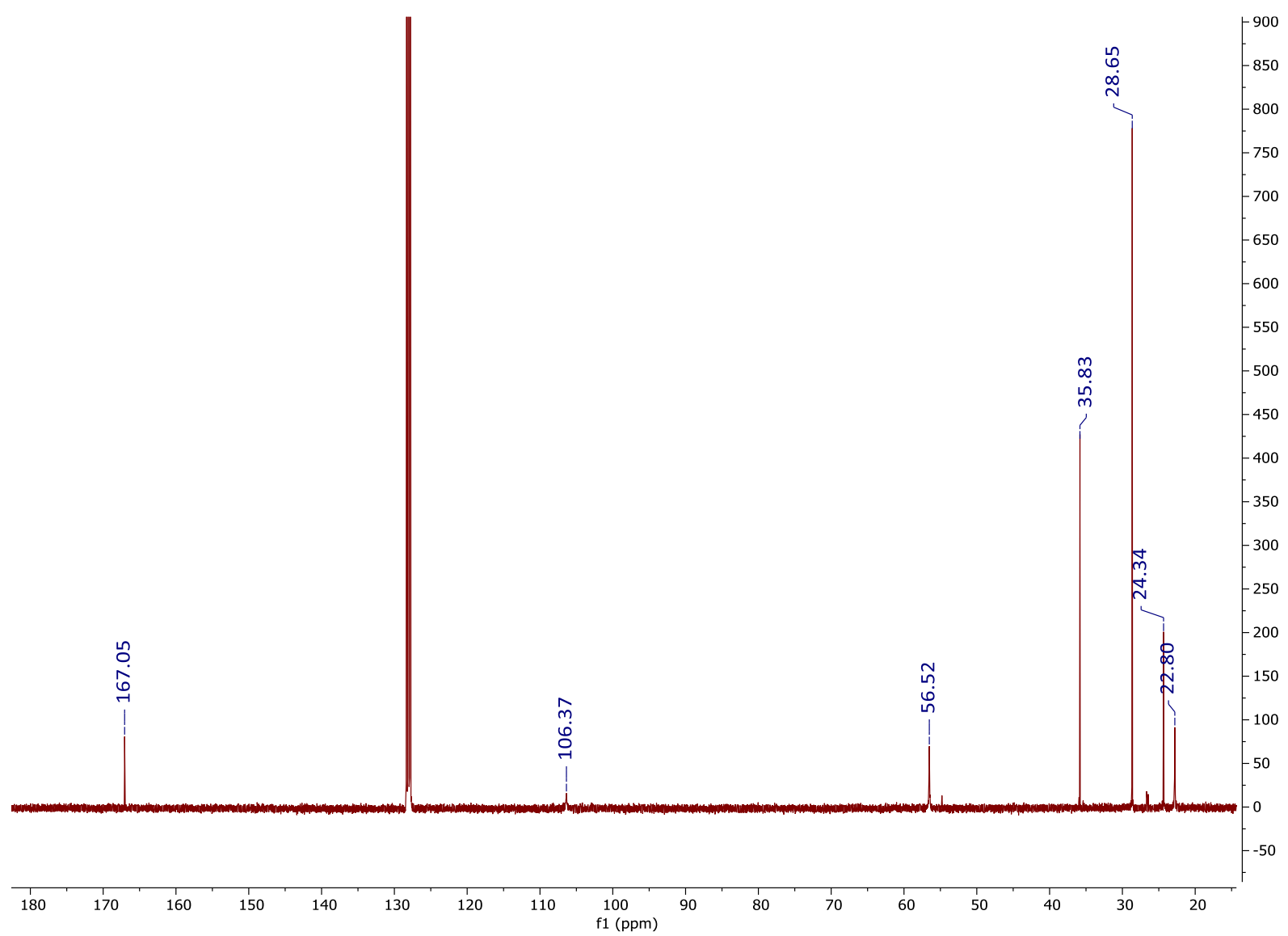


Figure S13. ${ }^{1} \mathrm{H}$ NMR Spectrum of 15 in $\mathrm{C}_{6} \mathrm{D}_{6}$ at $23{ }^{\circ} \mathrm{C}$.

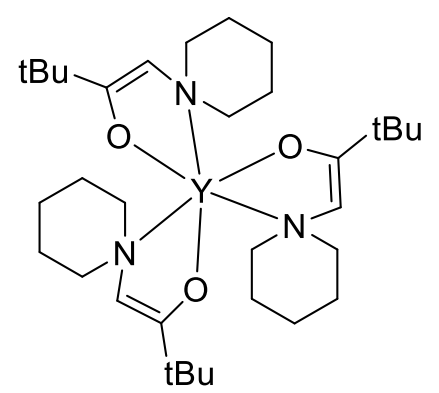

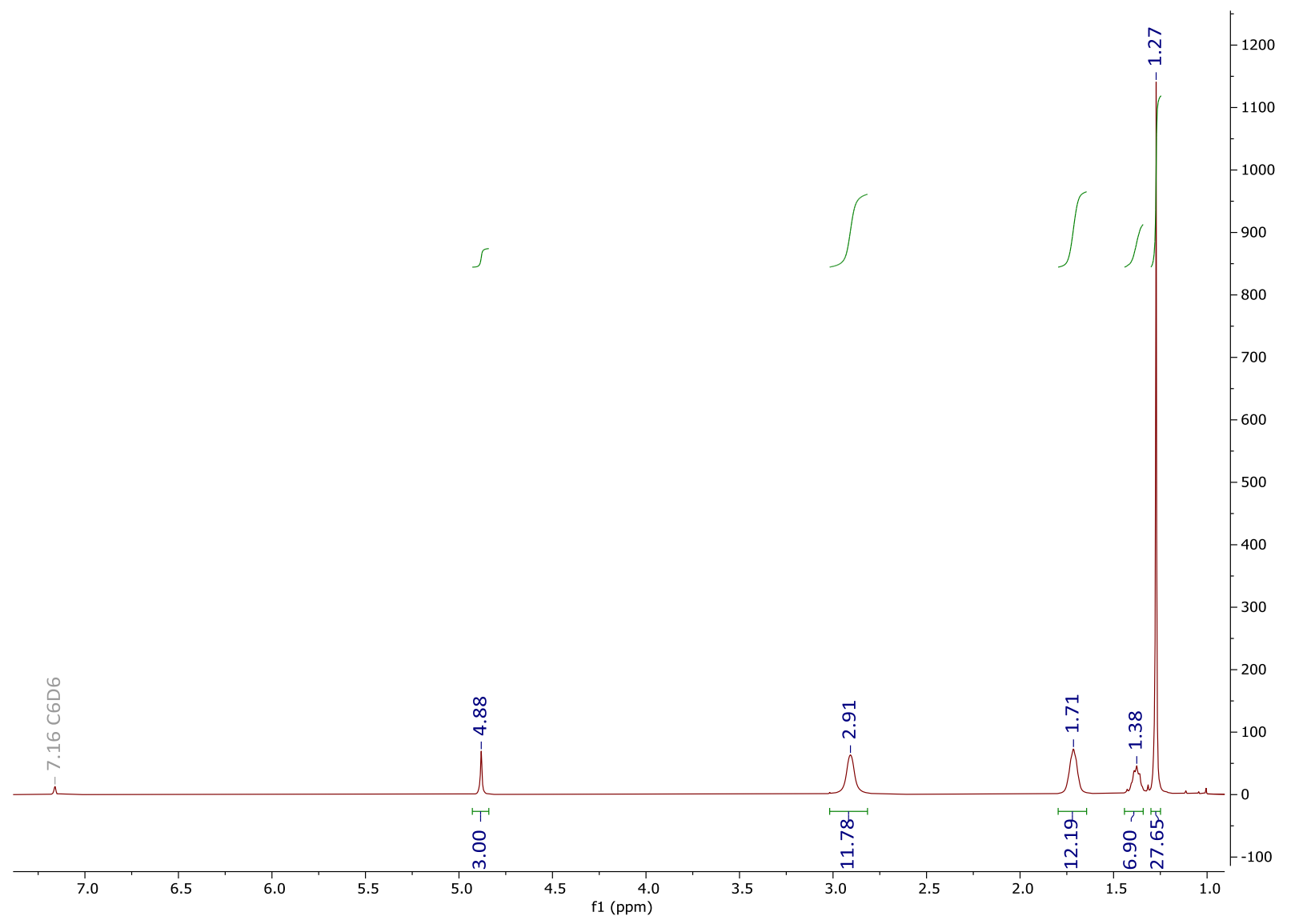


Figure S14. ${ }^{13} \mathrm{C}$ NMR Spectrum of 15 in $\mathrm{C}_{6} \mathrm{D}_{6}$ at $23{ }^{\circ} \mathrm{C}$.

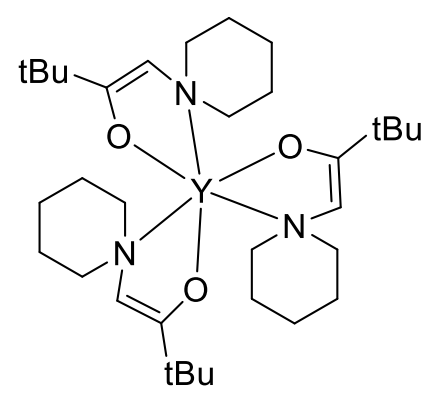

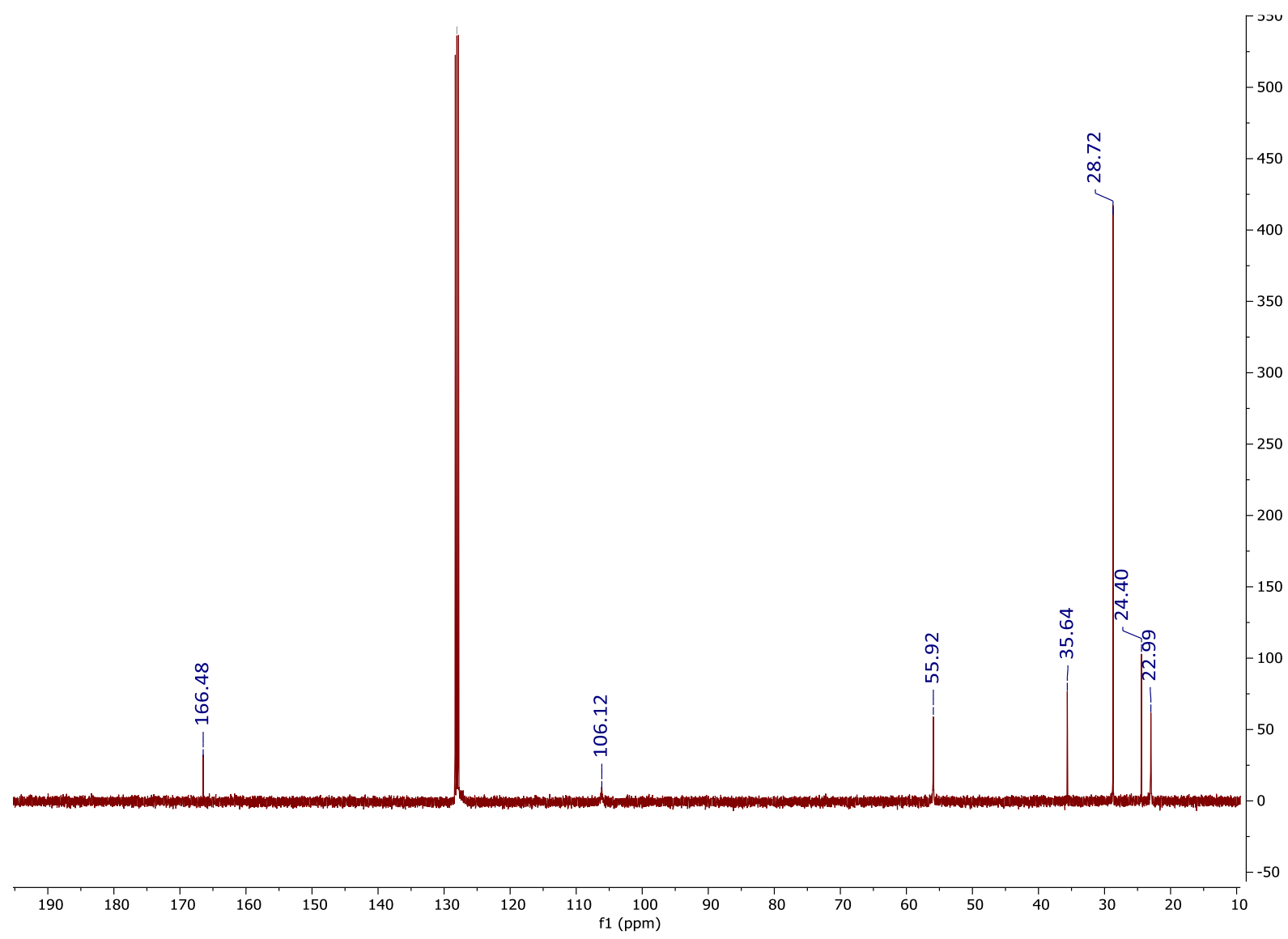


Figure S15. Variable Temperature ${ }^{1} \mathrm{H}$ NMR Spectra of $\mathbf{3}$ in Toluene- $d_{8}$.
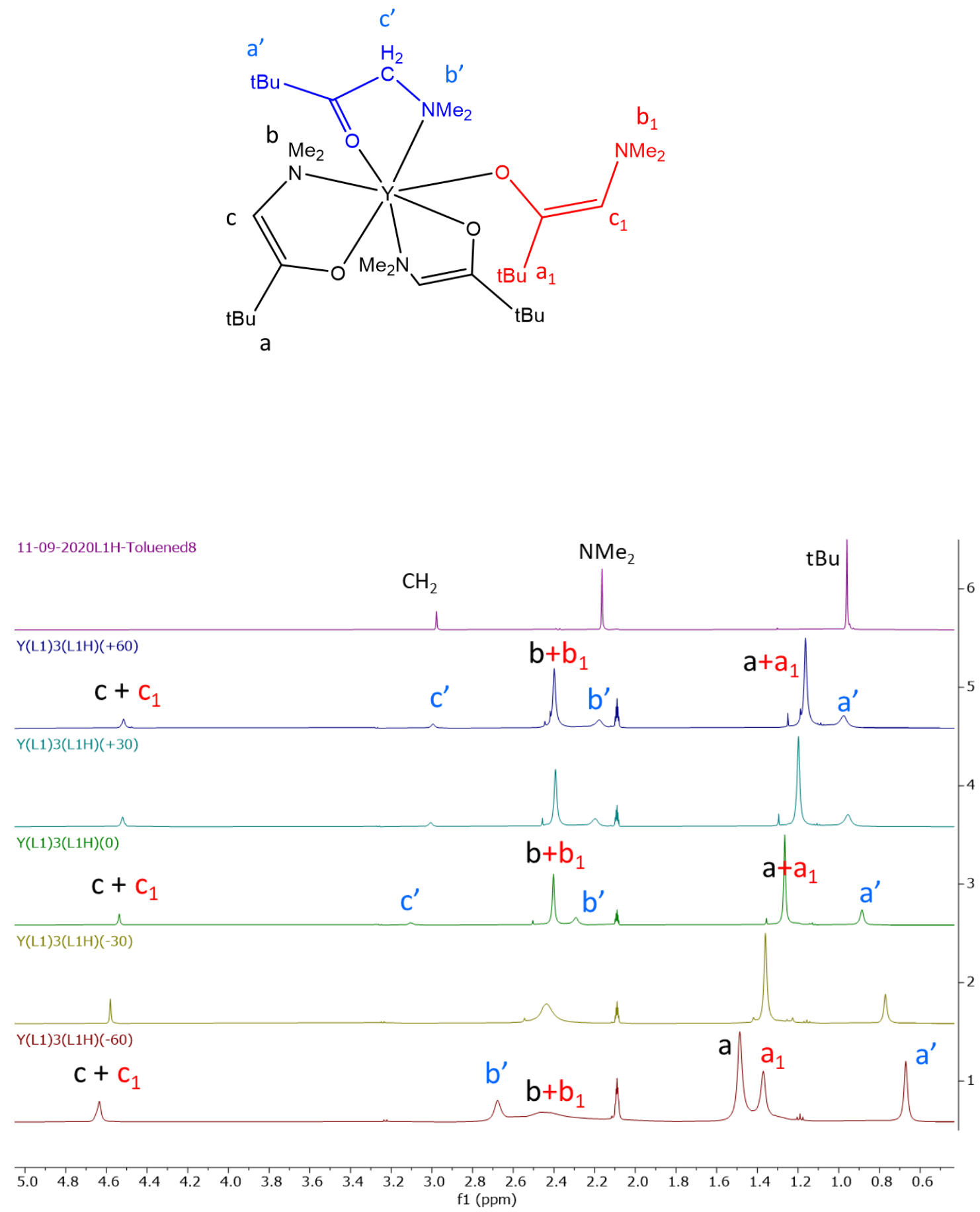
Figure S16. Variable Temperature ${ }^{1} \mathrm{H}$ NMR Spectra of 6 in Toluene- $d_{8}$.
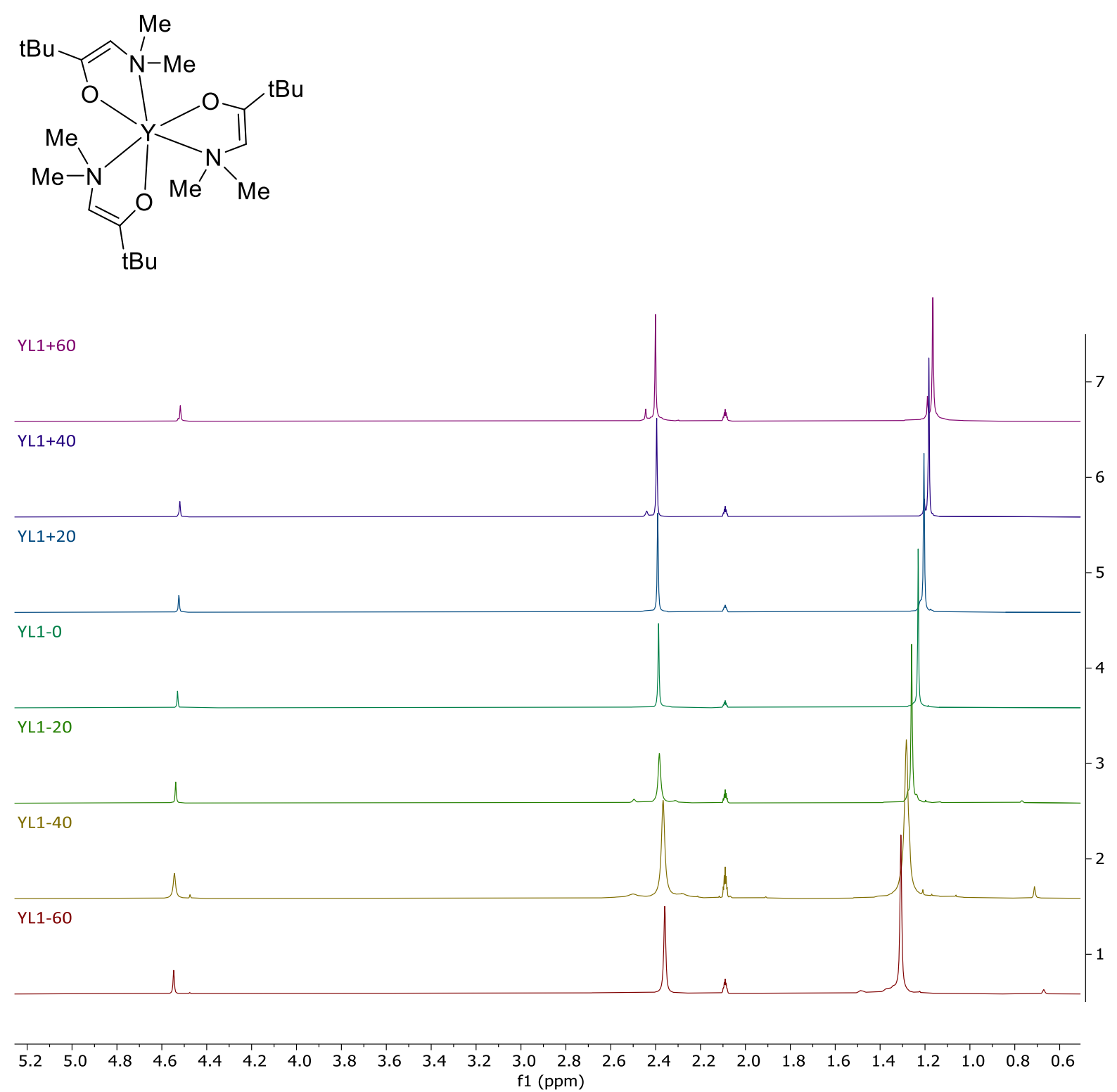
Figure S17. Infrared Spectrum of 1.

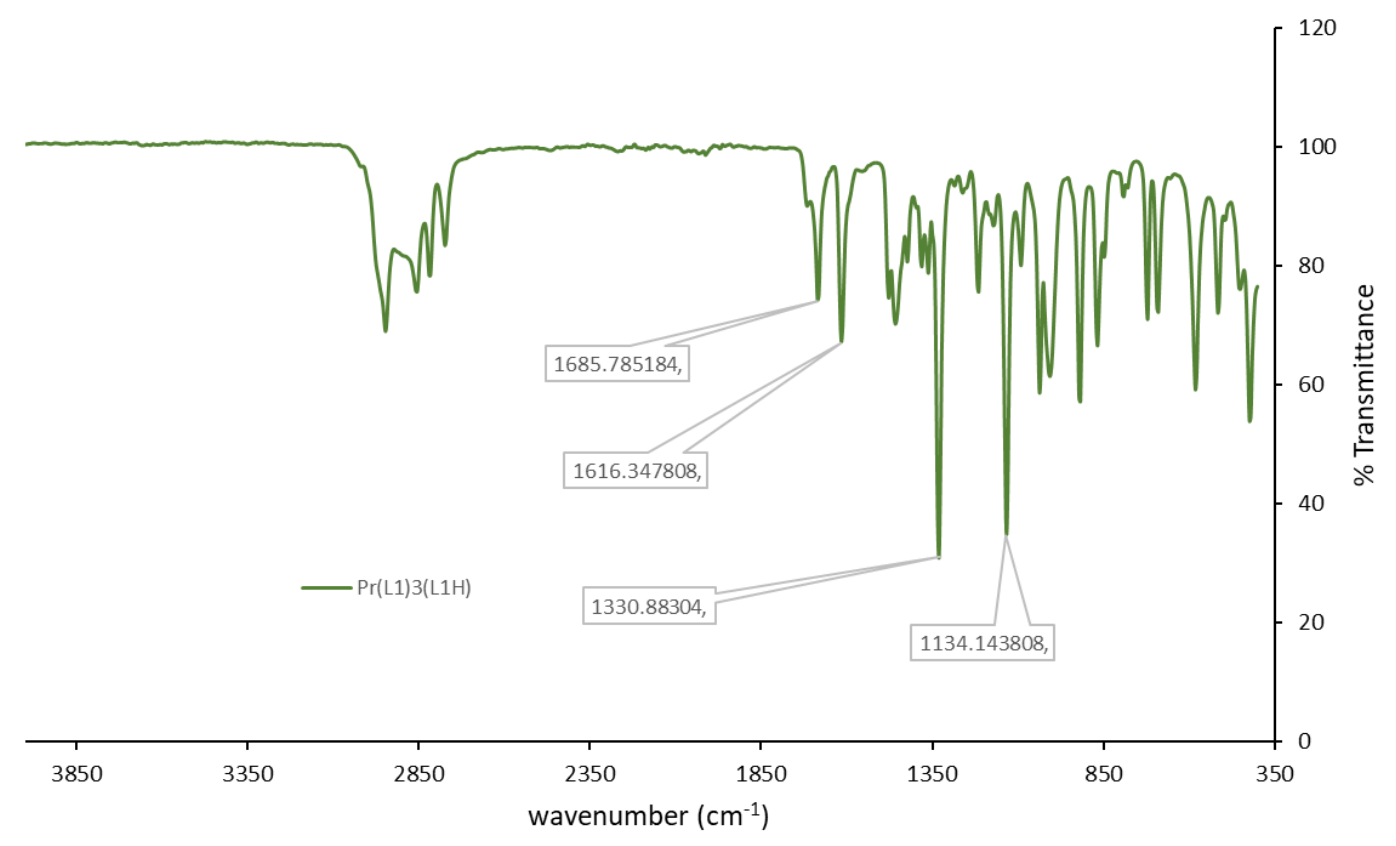

Figure S18. Infrared Spectrum of 2.

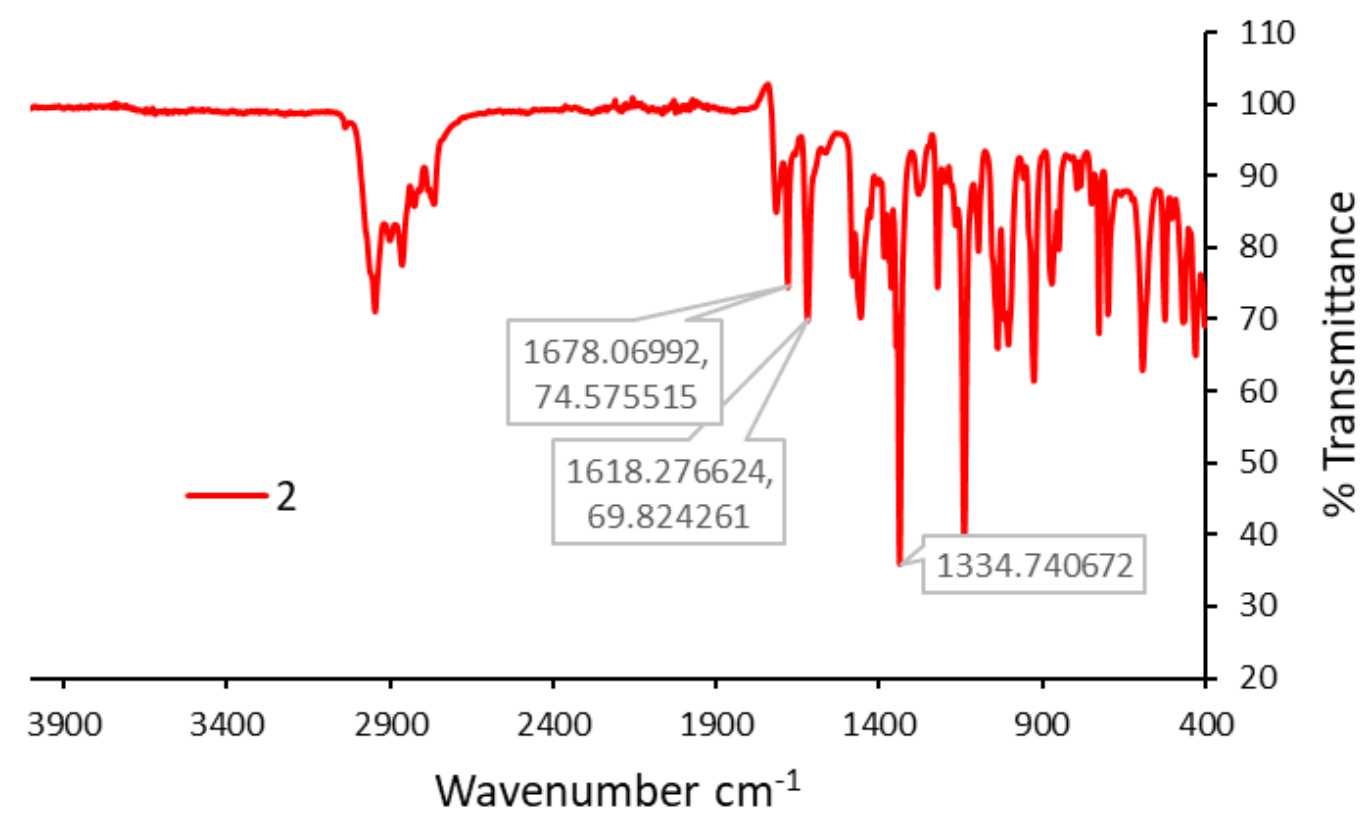


Figure S19. Infrared Spectrum of 3.

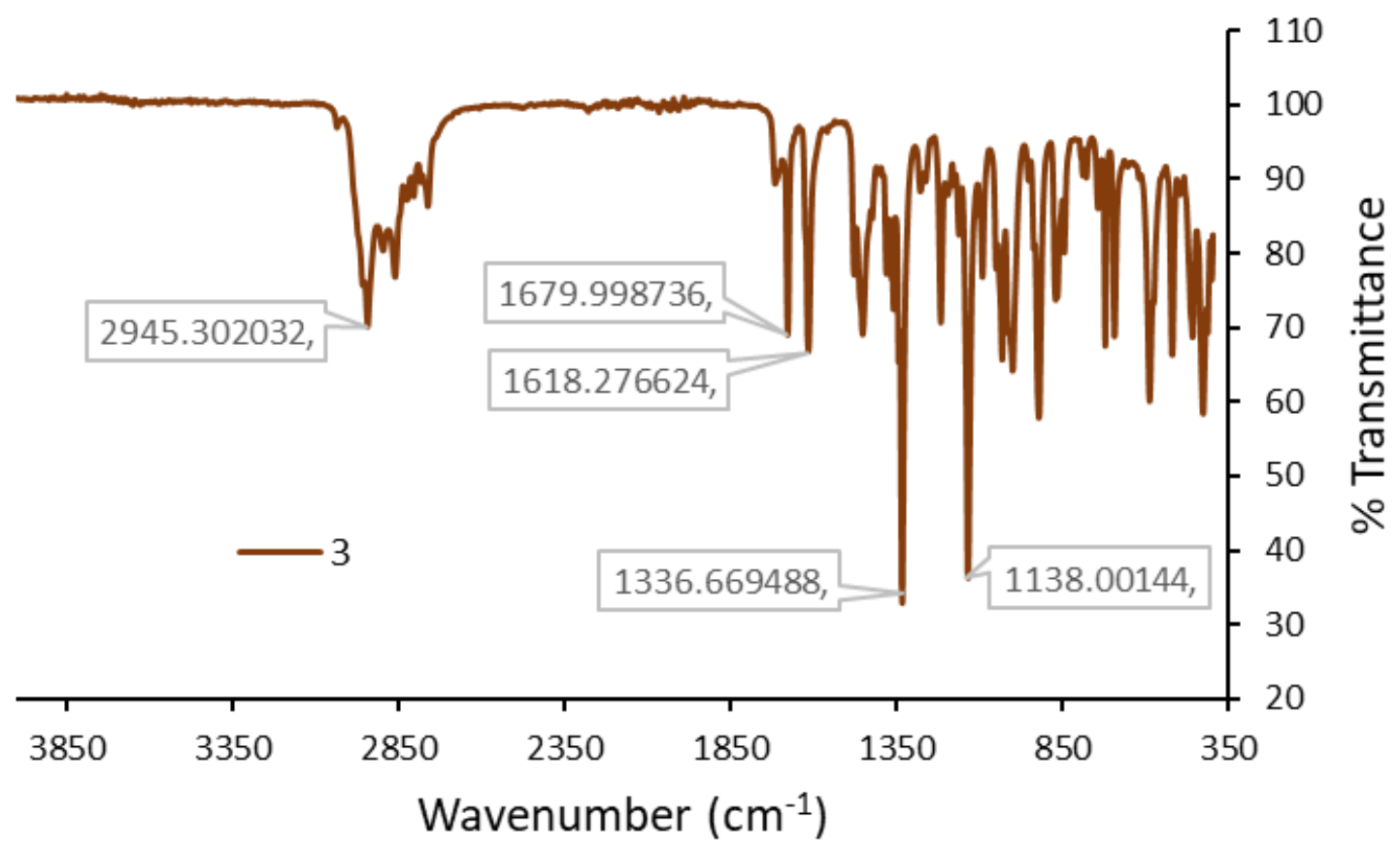

Figure S20. Infrared Spectrum of 4.

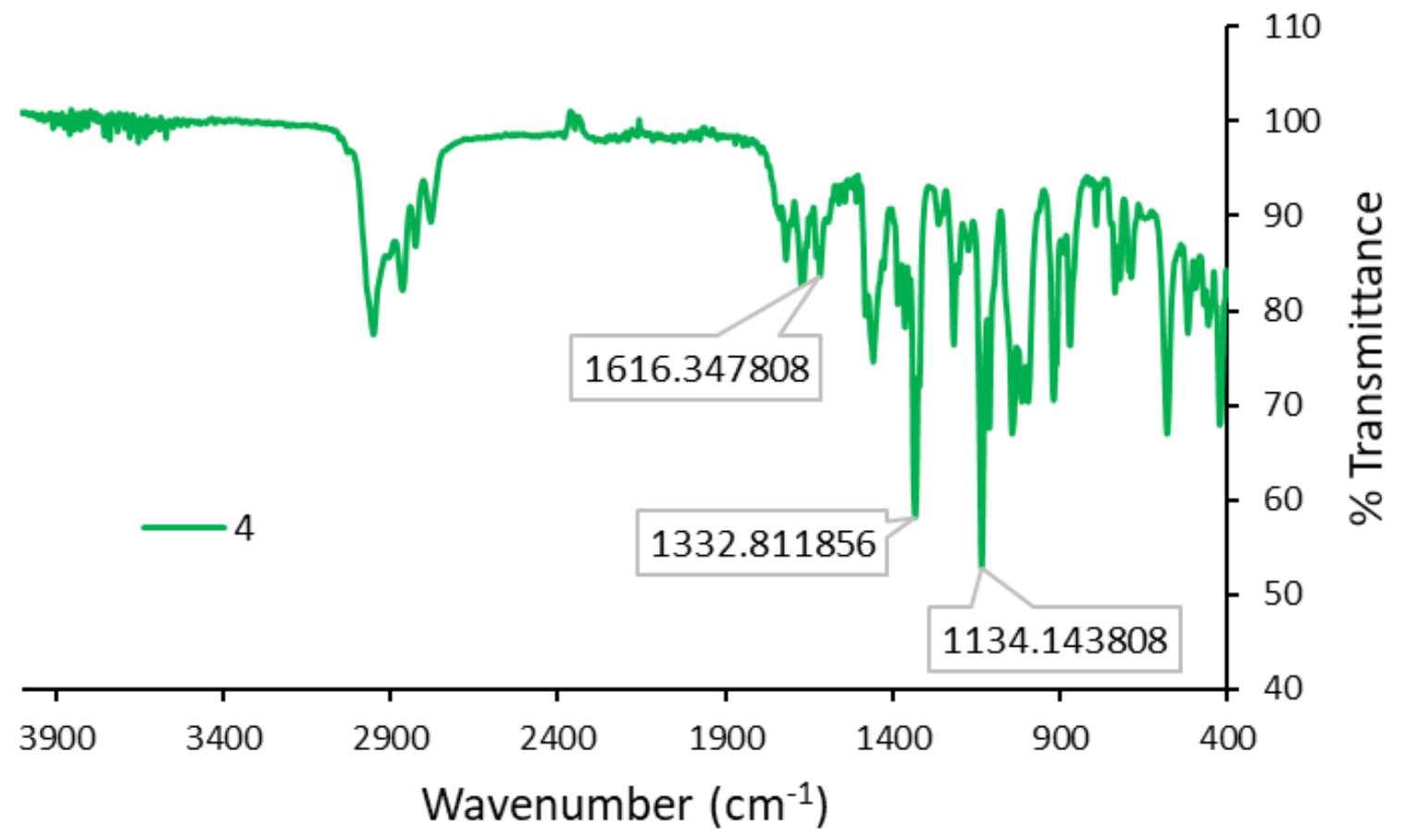


Figure S21. Infrared Spectrum of 5.

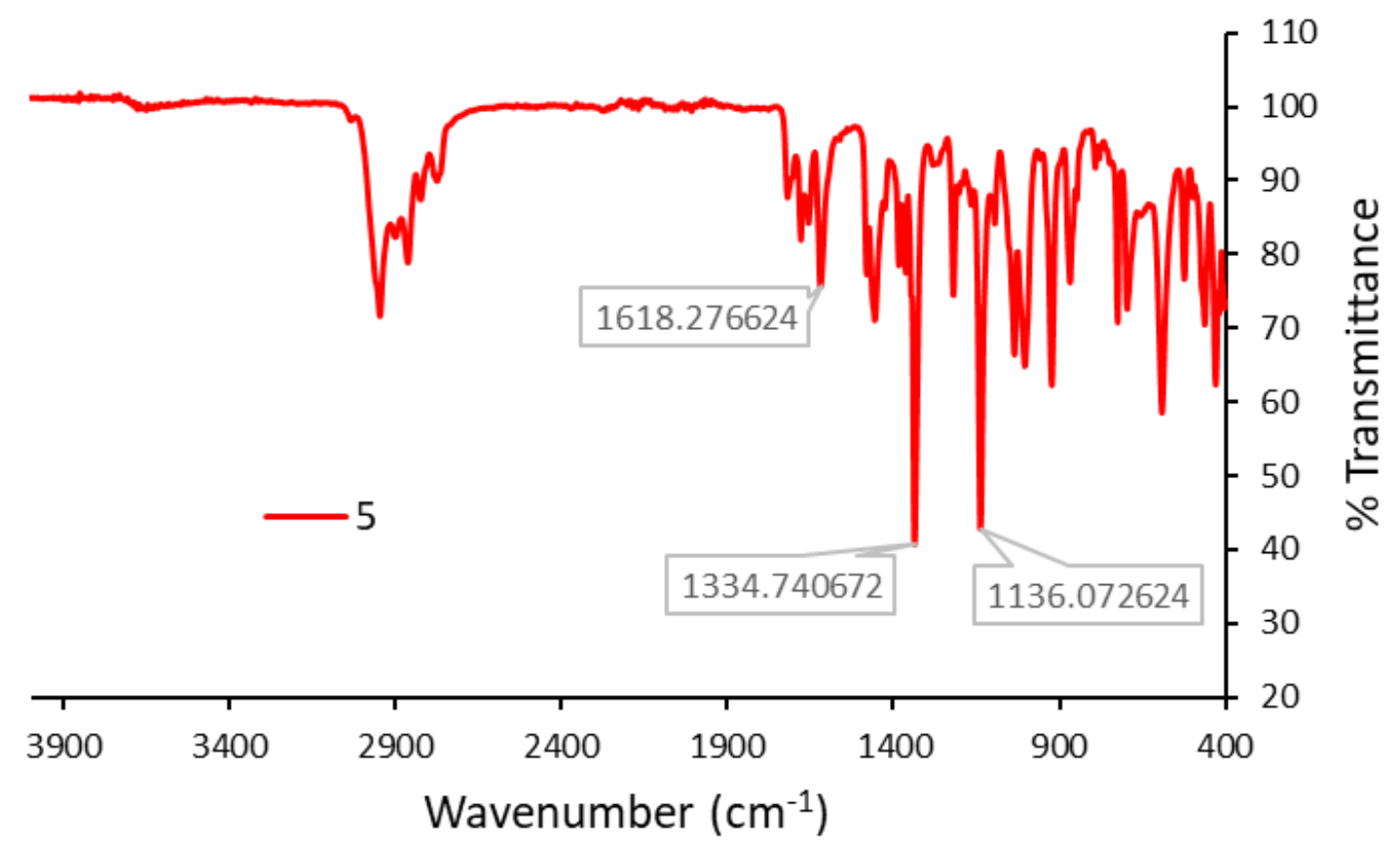

Figure S22. Infrared Spectrum of 6.

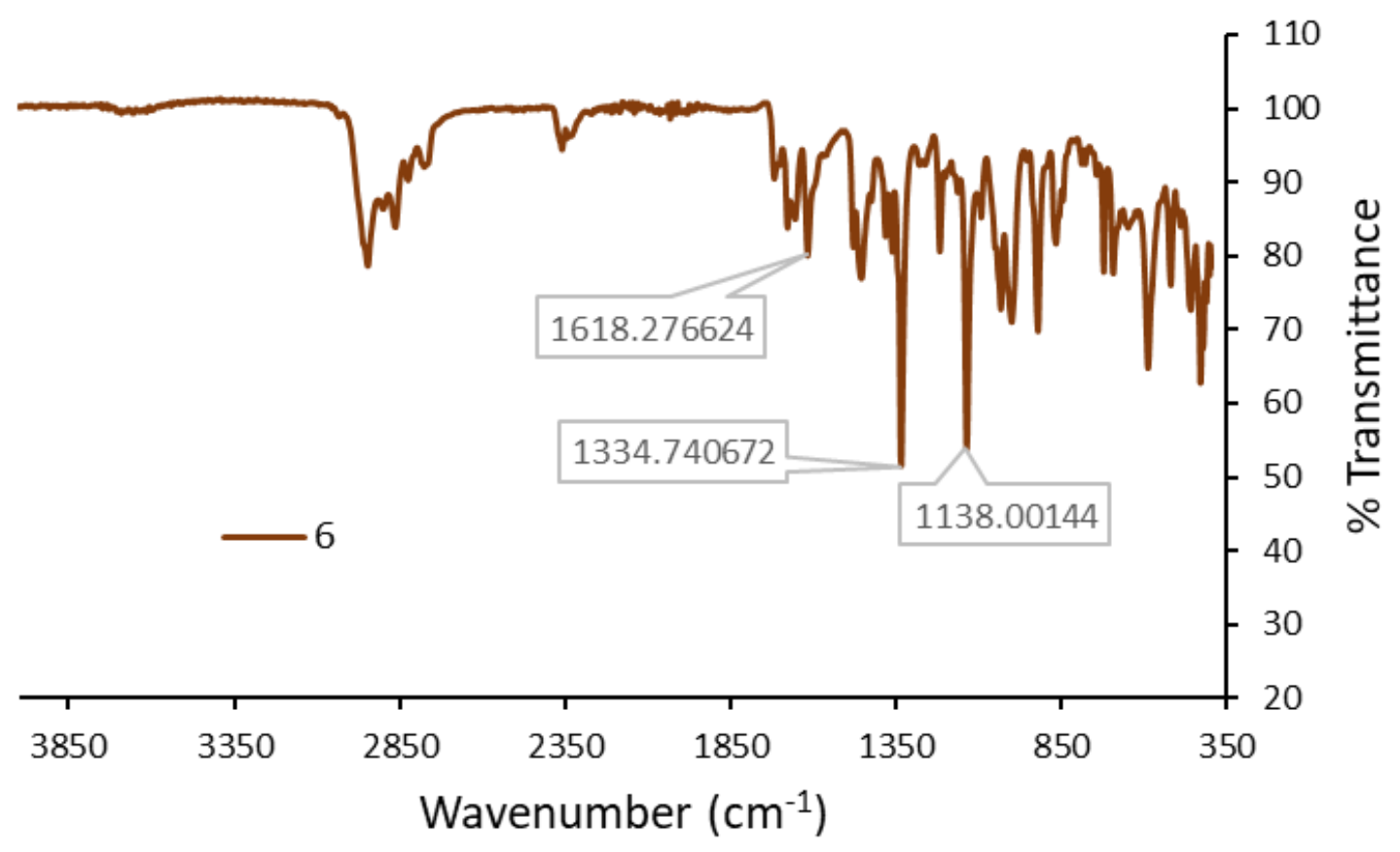


Figure S23. Infrared Spectrum of 7.

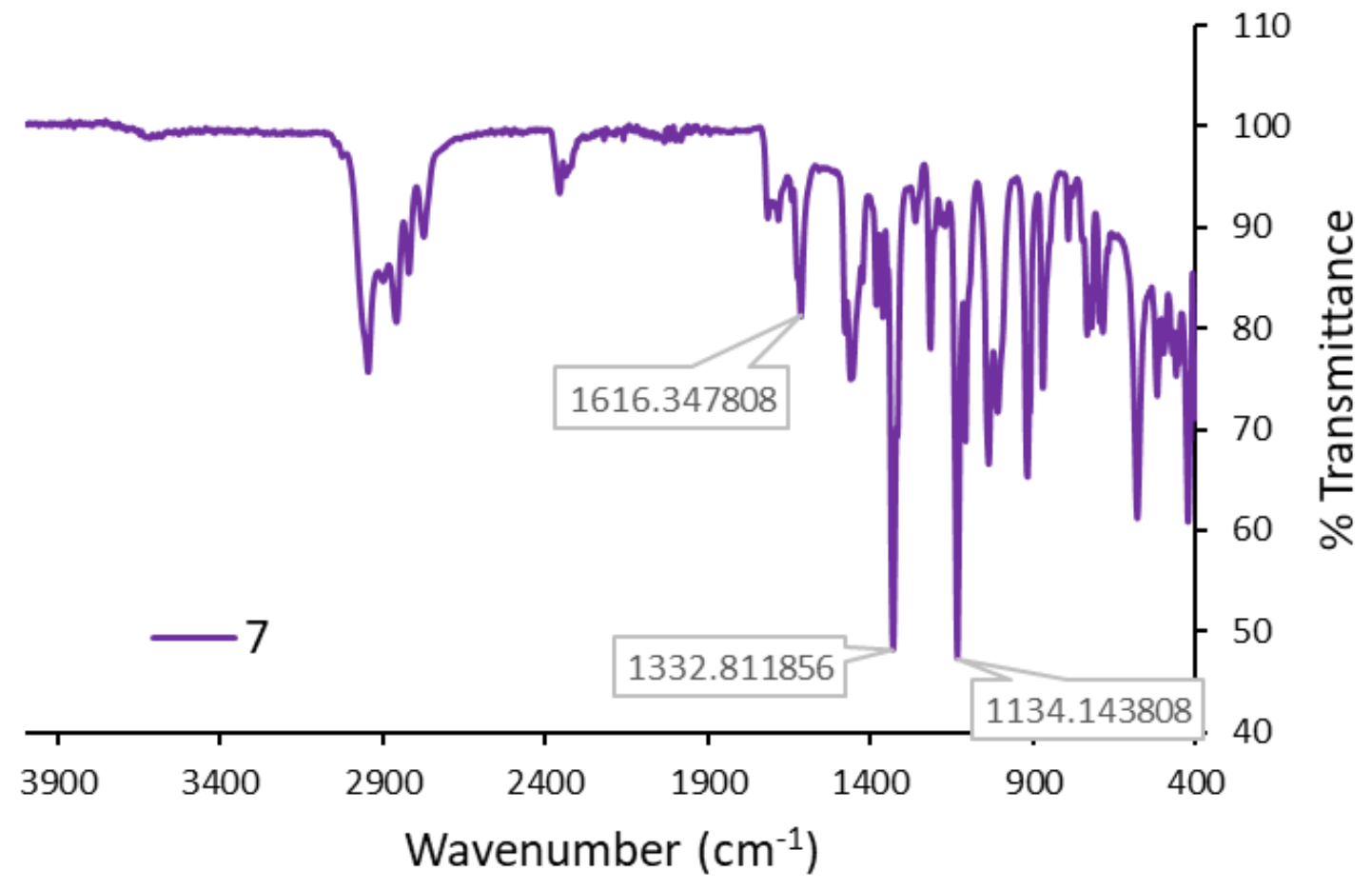

Figure S24. Infrared Spectrum of 8.

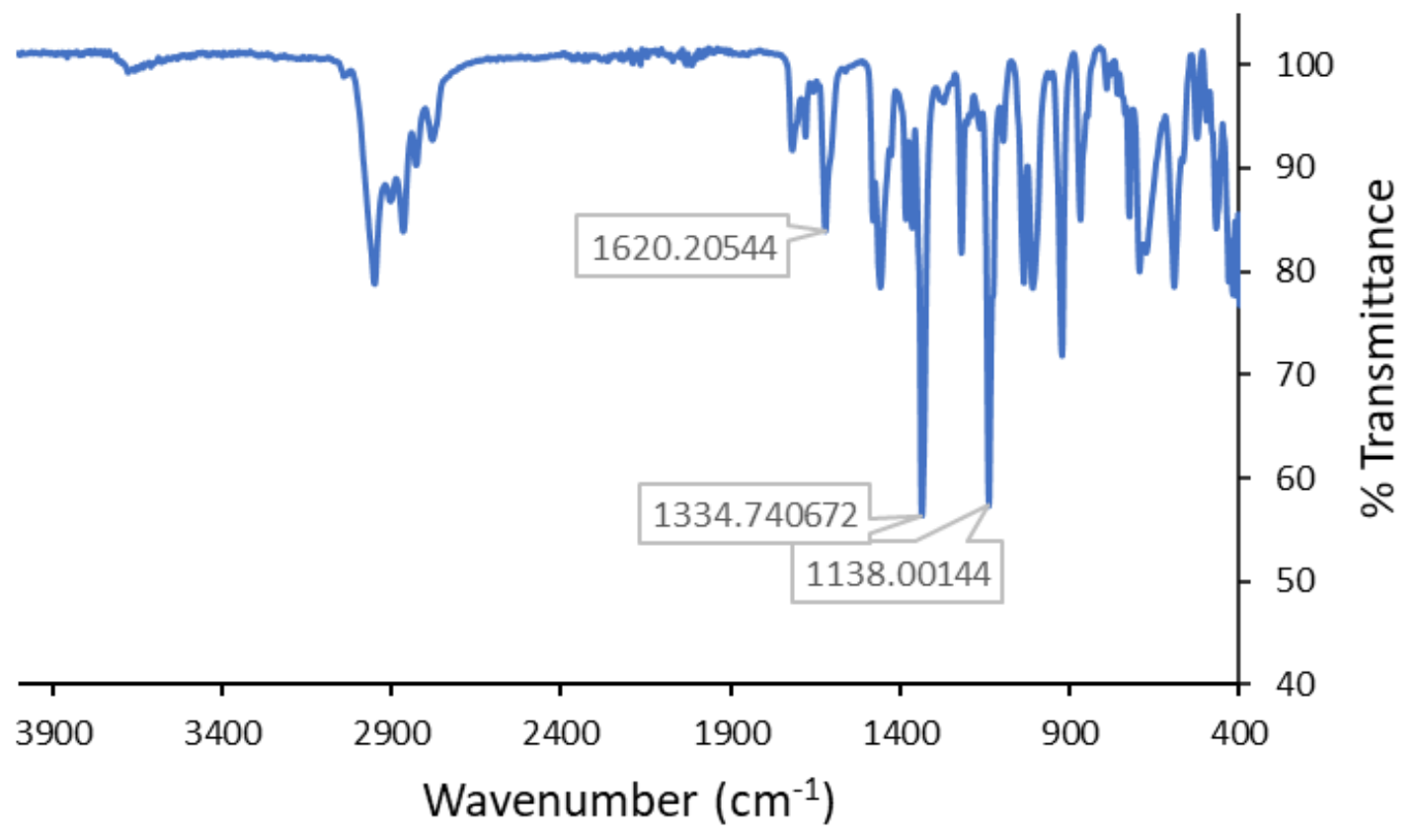


Figure S25. Infrared Spectrum of 9.

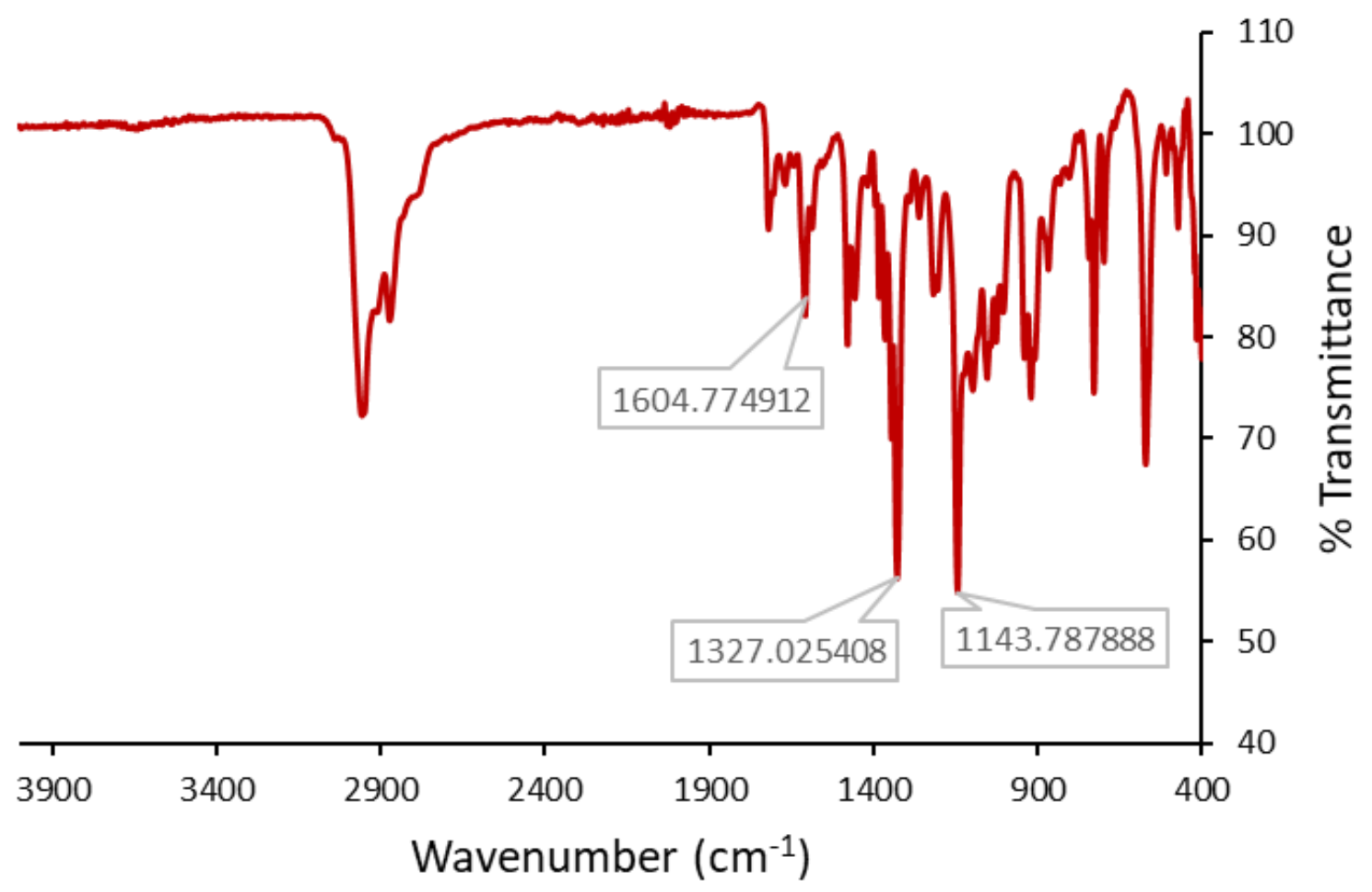

Figure S26. Infrared Spectrum of 10.

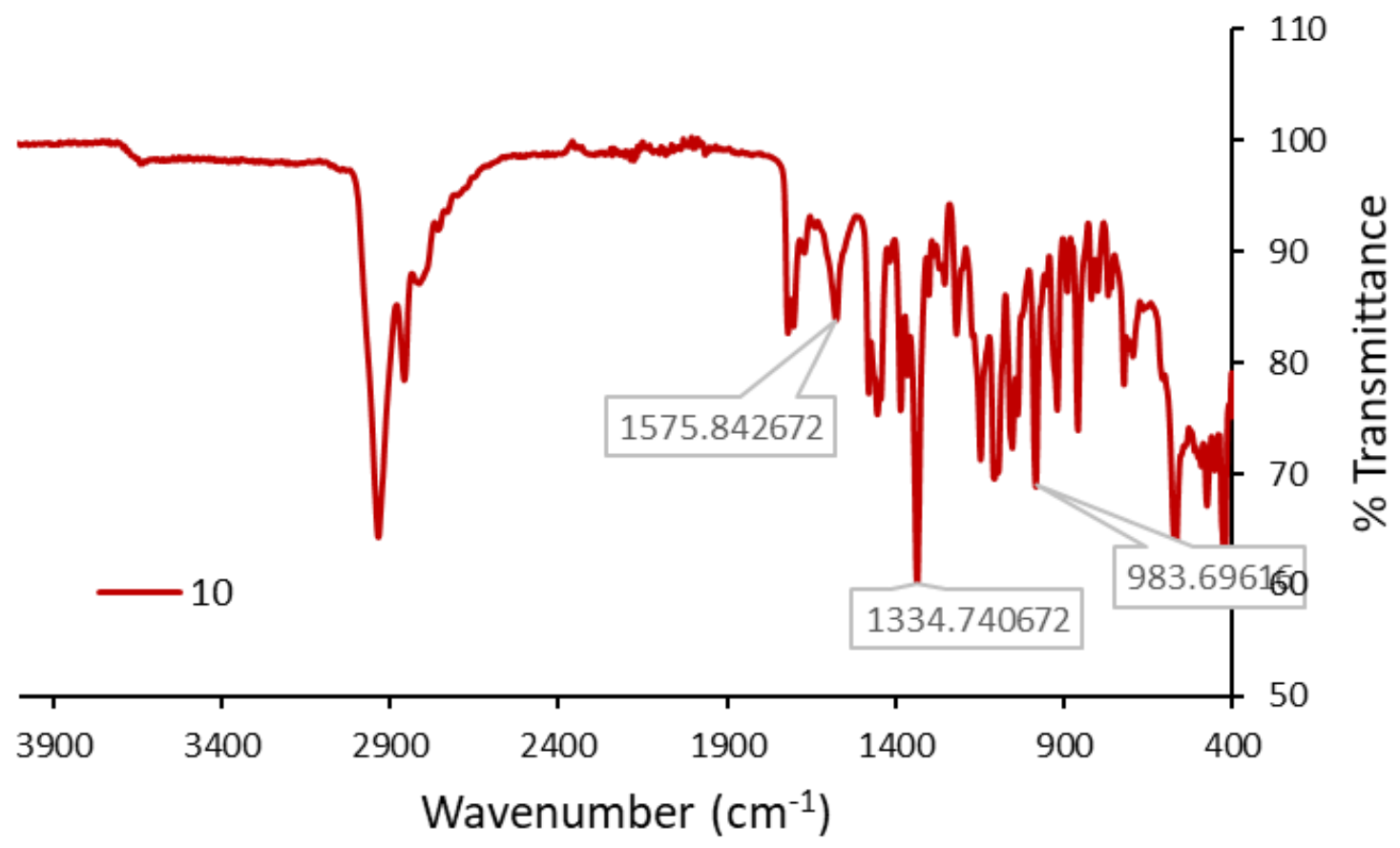


Figure S27. Infrared Spectrum of 11.

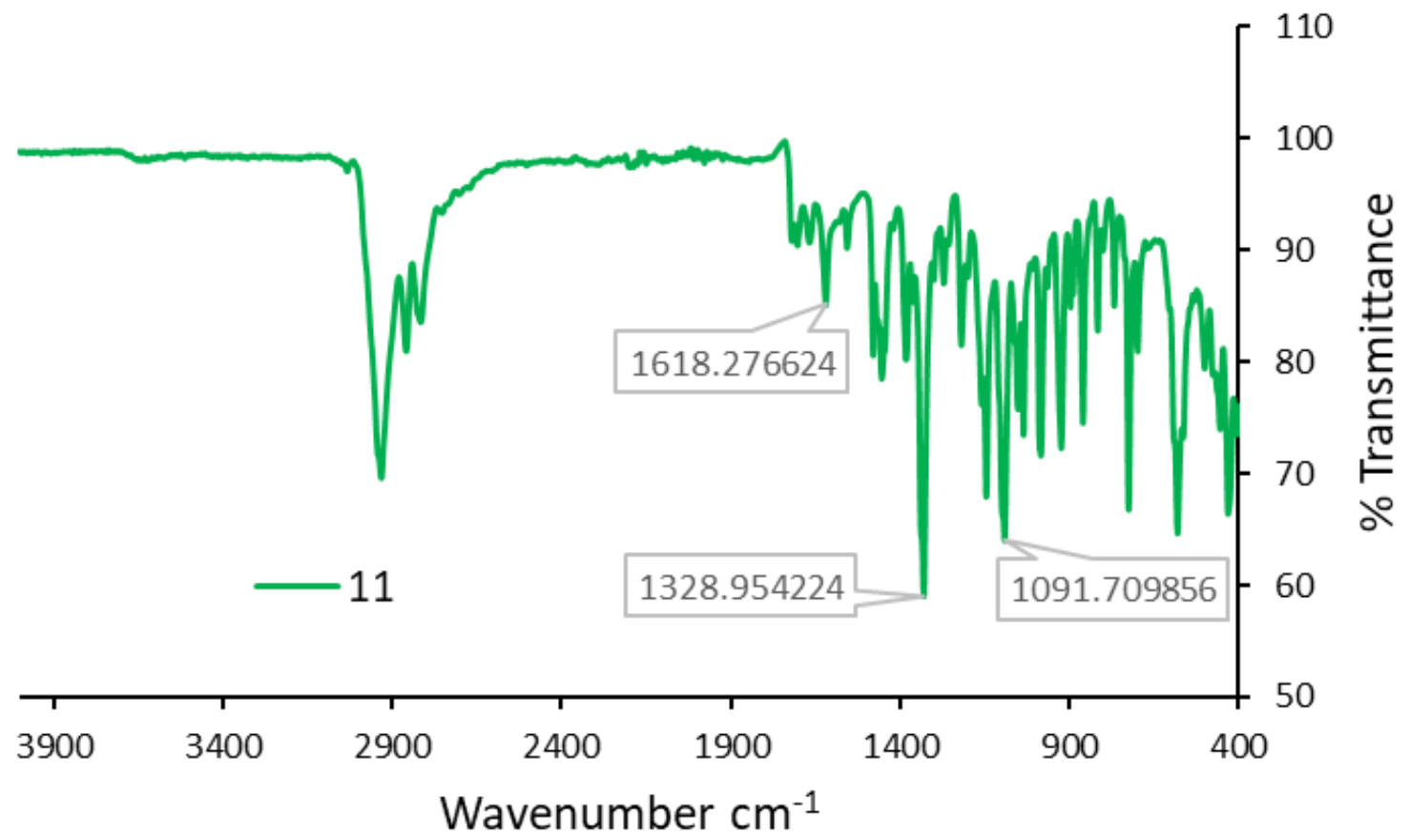

Figure S28. Infrared Spectrum of 12.

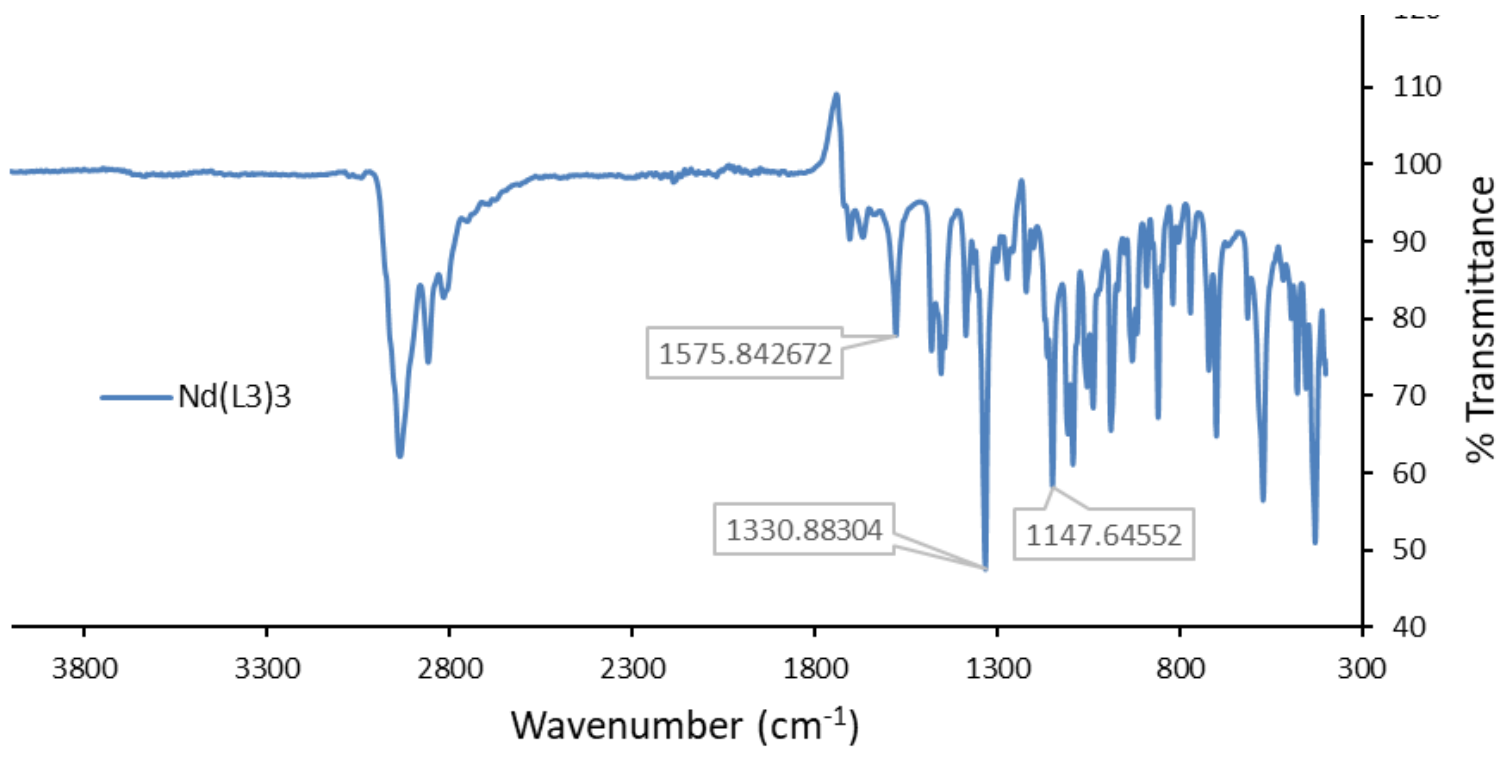


Figure S29. Infrared Spectrum of 13.

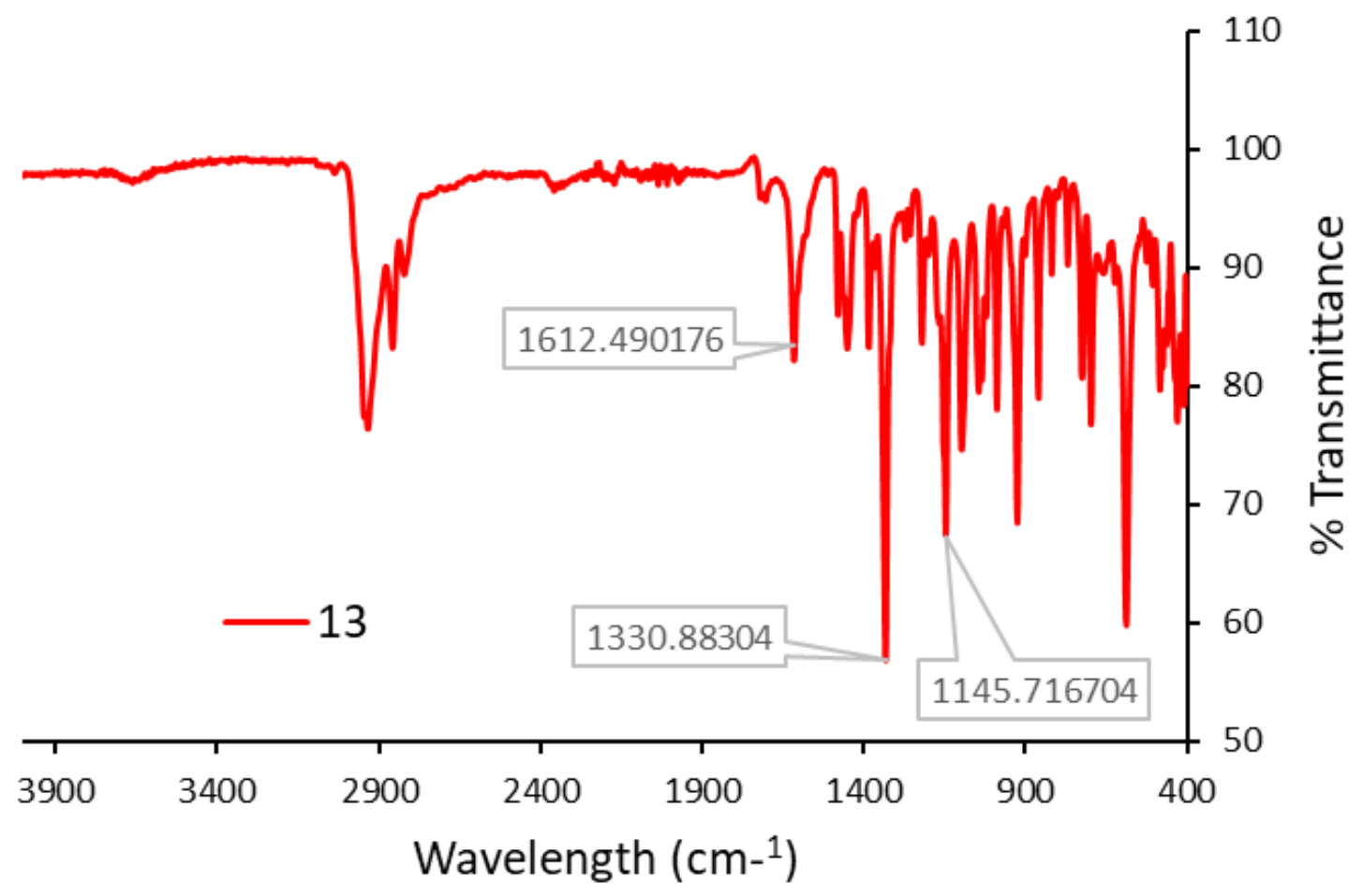

Figure S30. Infrared Spectrum of 14.

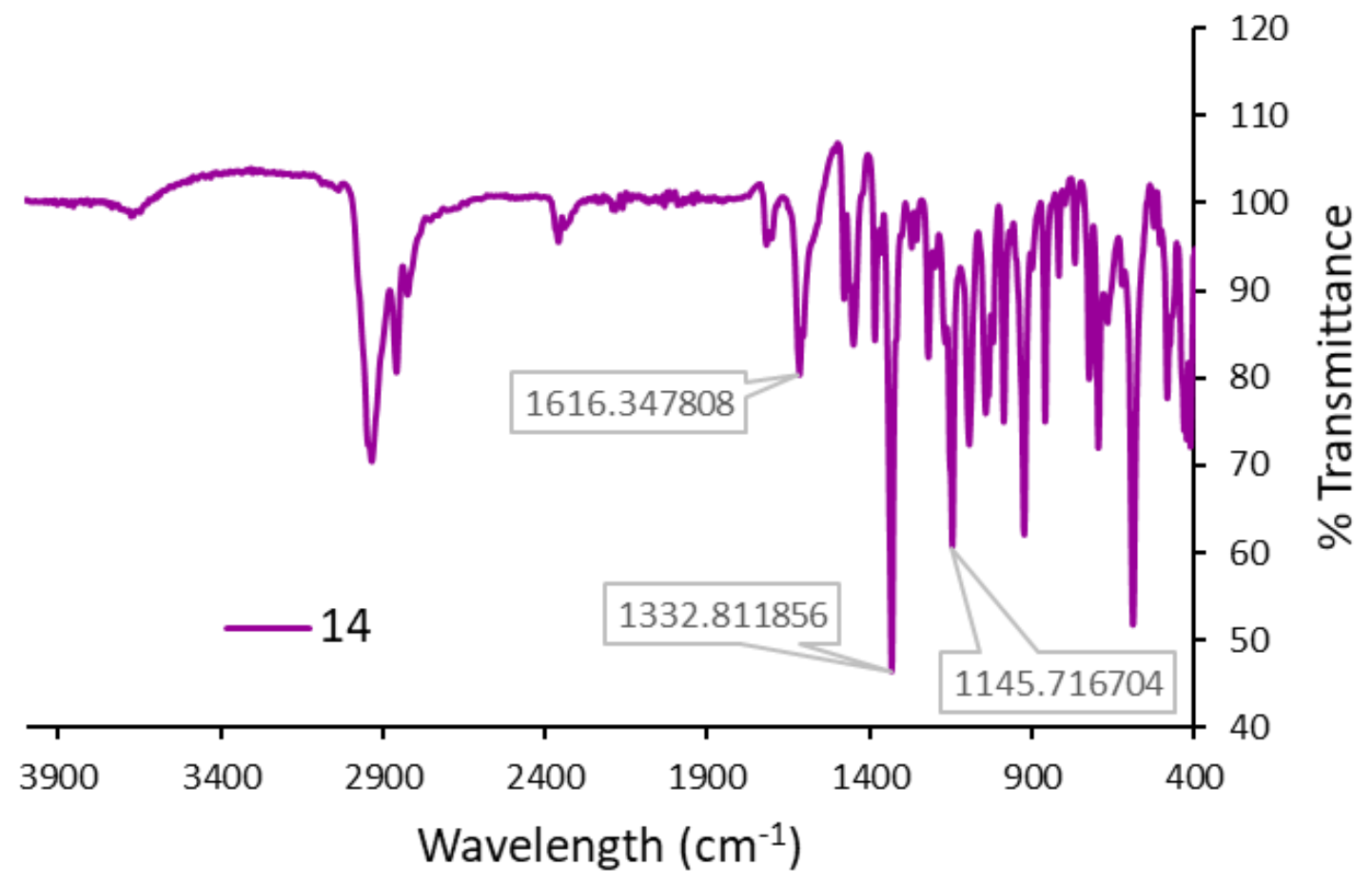


Figure S31. Infrared Spectrum of 15.

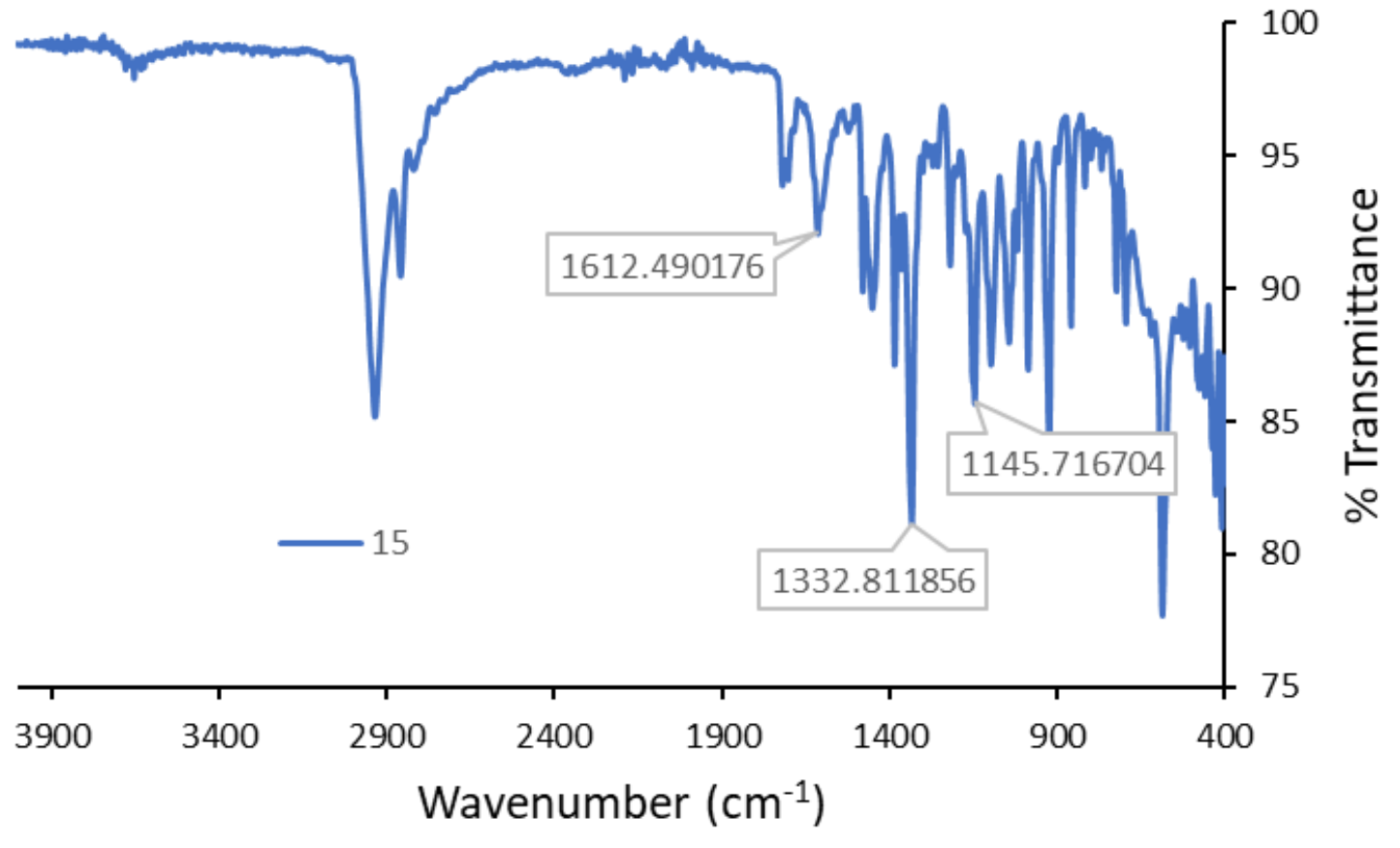

Figure S32. TGA traces for 7-9.

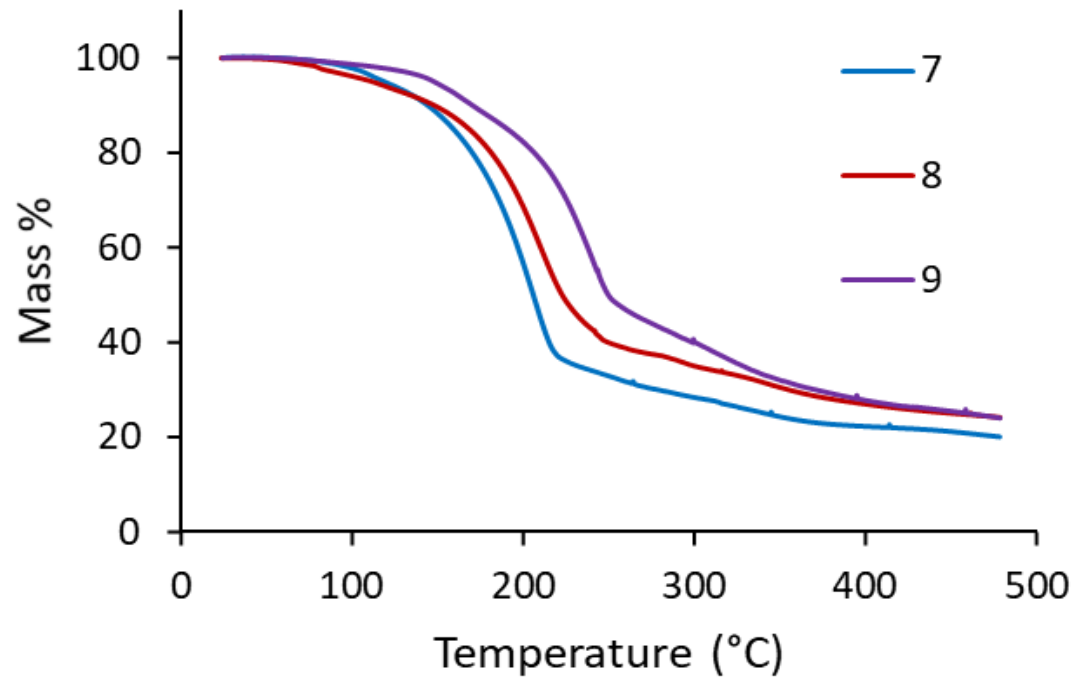


Figure S33. TGA traces for 10-12.

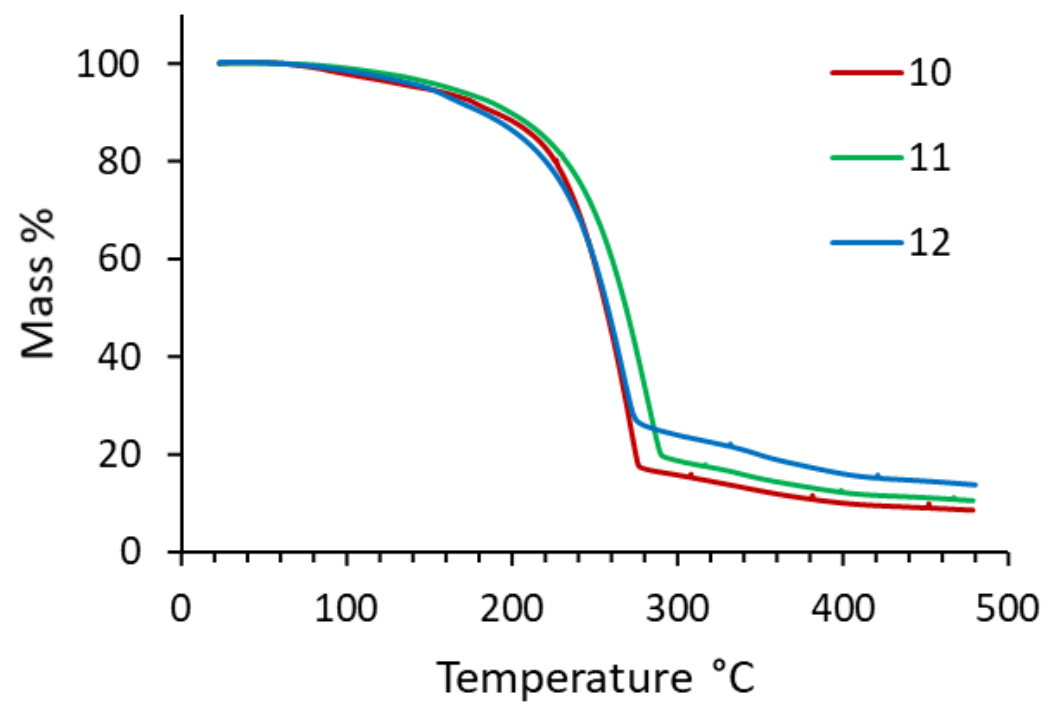


Figure S34. ${ }^{1} \mathrm{H}$ NMR spectra of 6 (a) pure sublimed sample and (b) after heating at $130{ }^{\circ} \mathrm{C}$ for 24 hours.

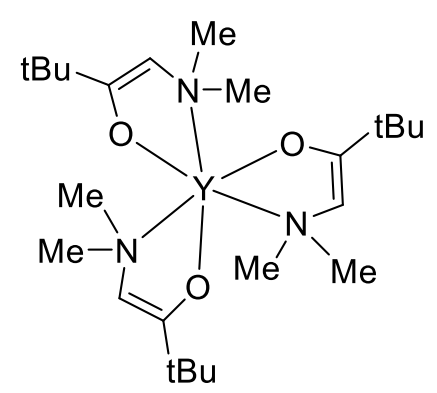

(a)

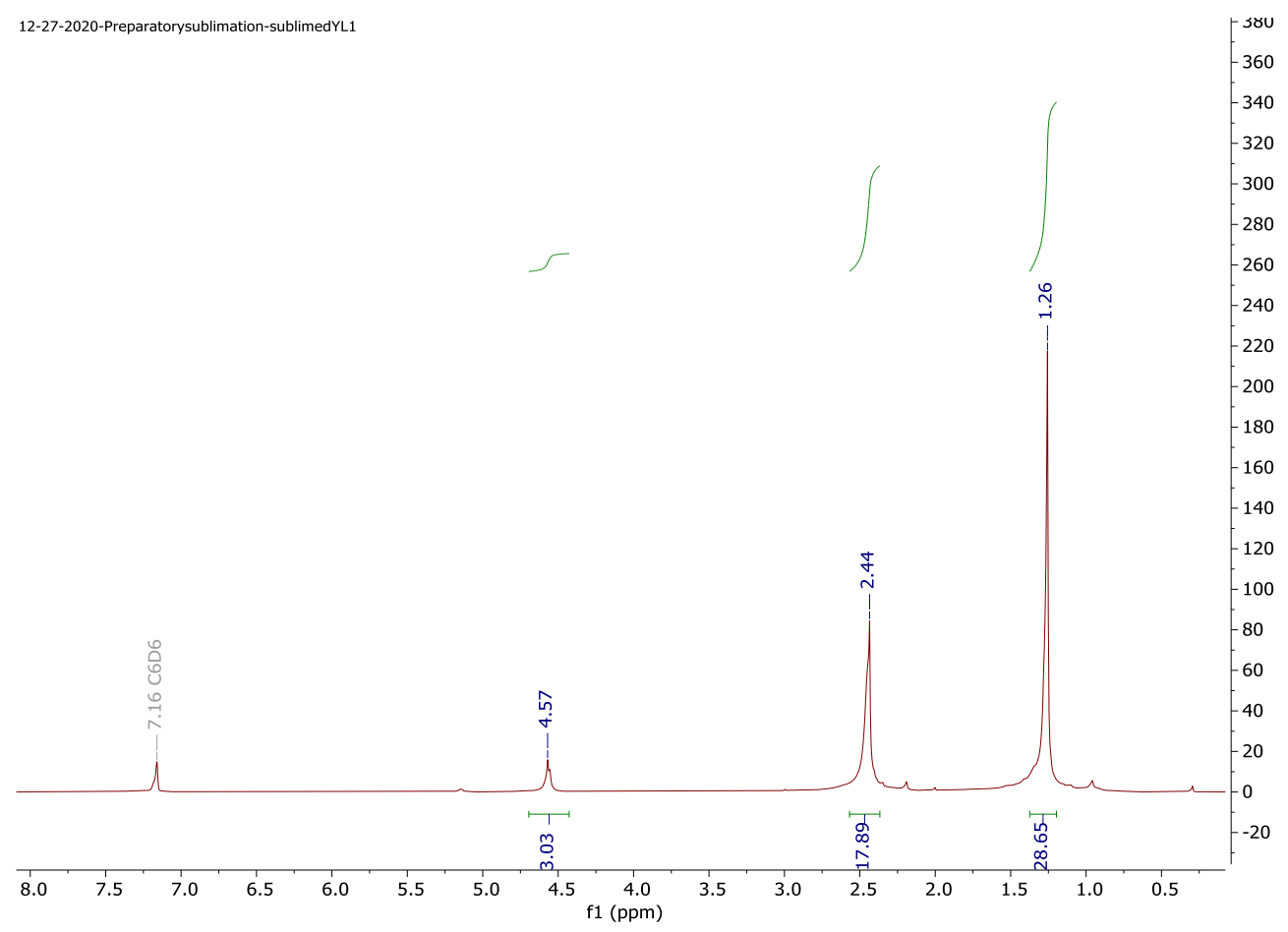


(b)

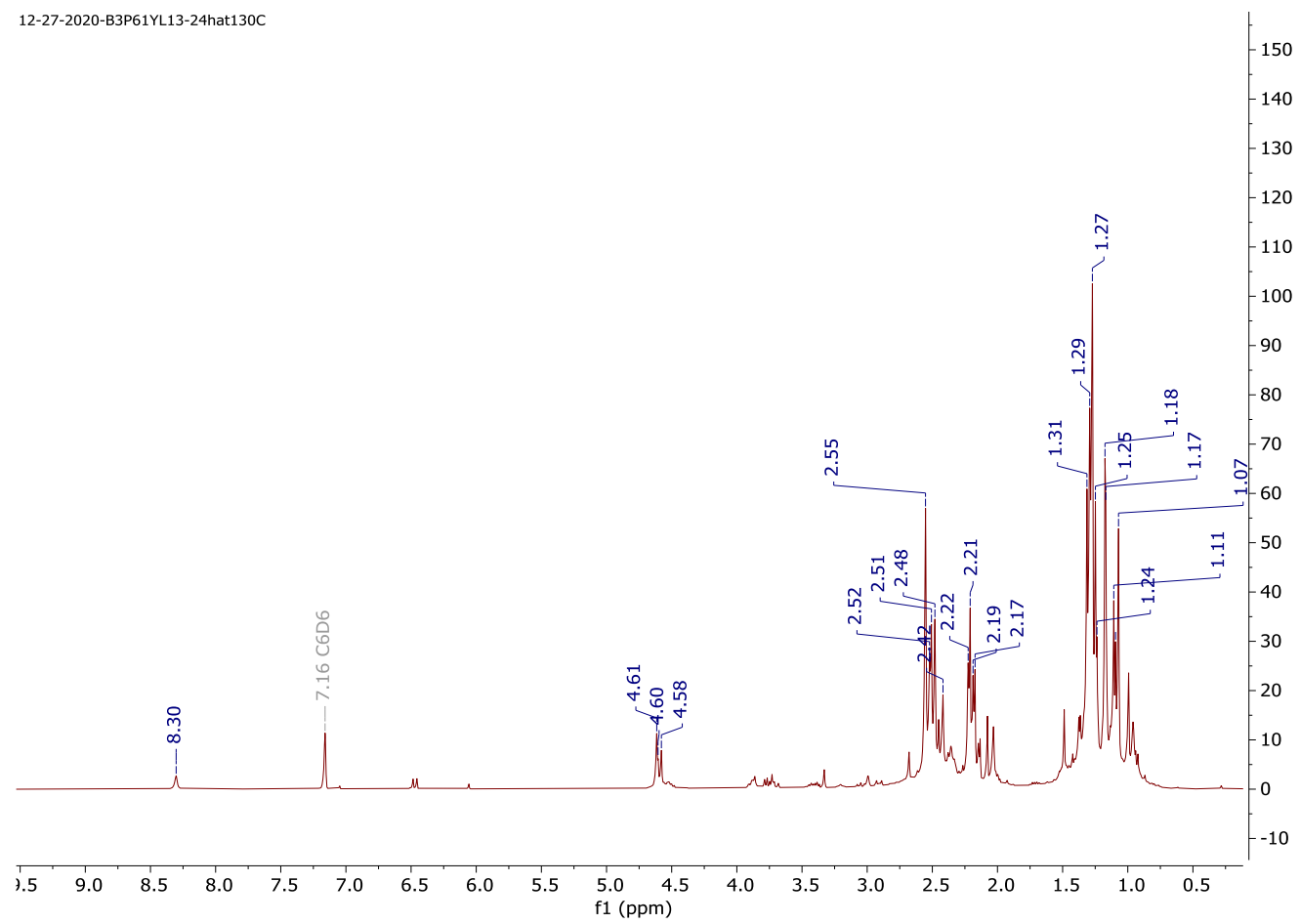


Figure S35. ${ }^{1} \mathrm{H}$ NMR spectra of 10 (a) pure sublimed sample and (b) after heating at $150{ }^{\circ} \mathrm{C}$ for 24 hours.

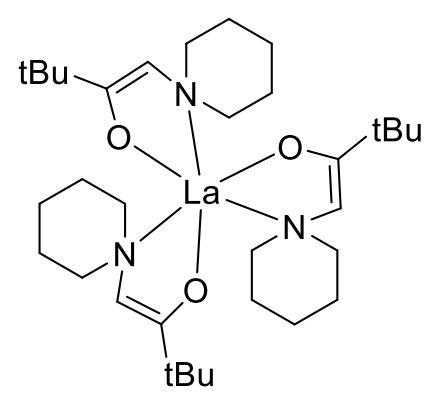

(a)

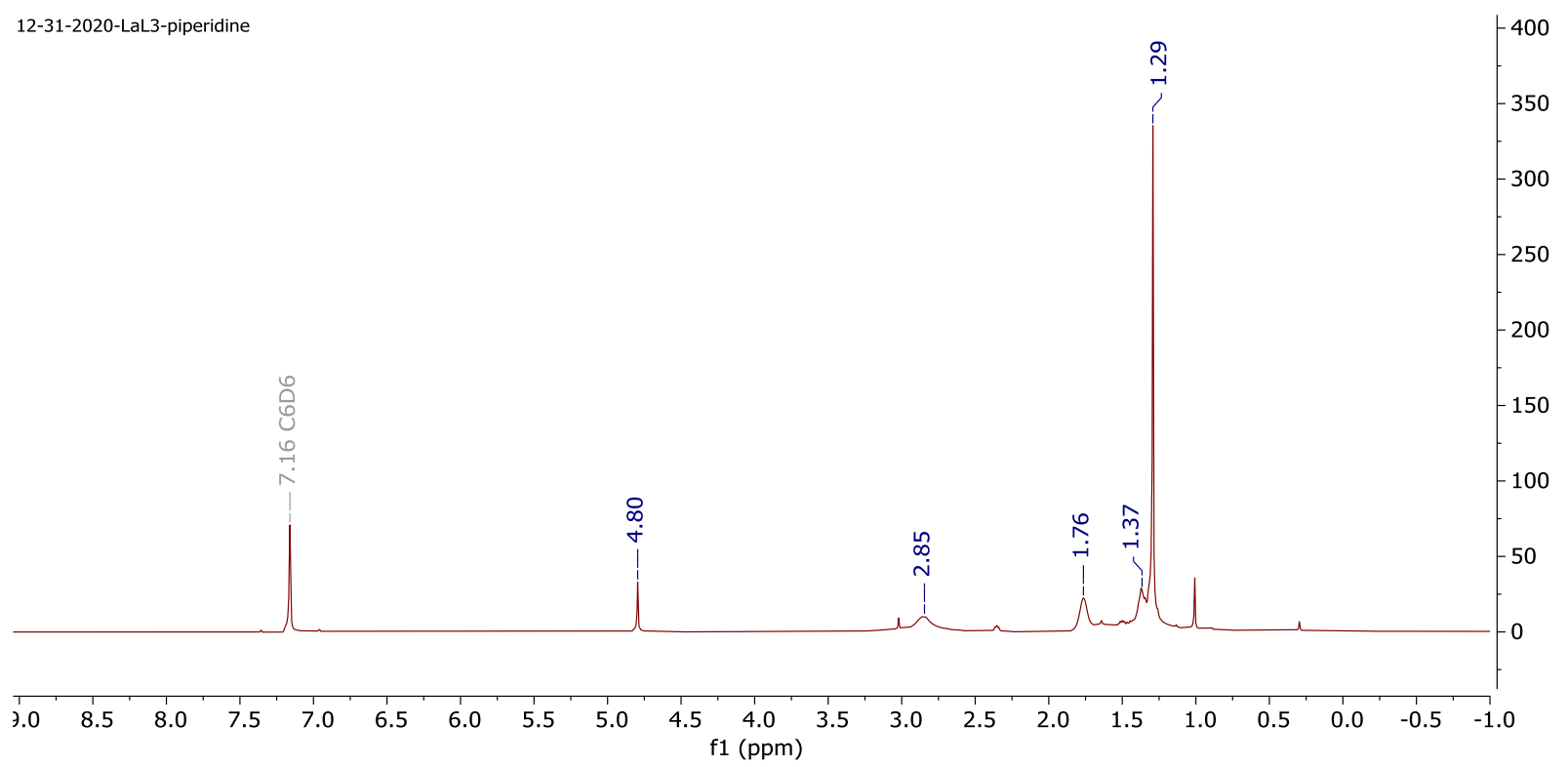


(b)

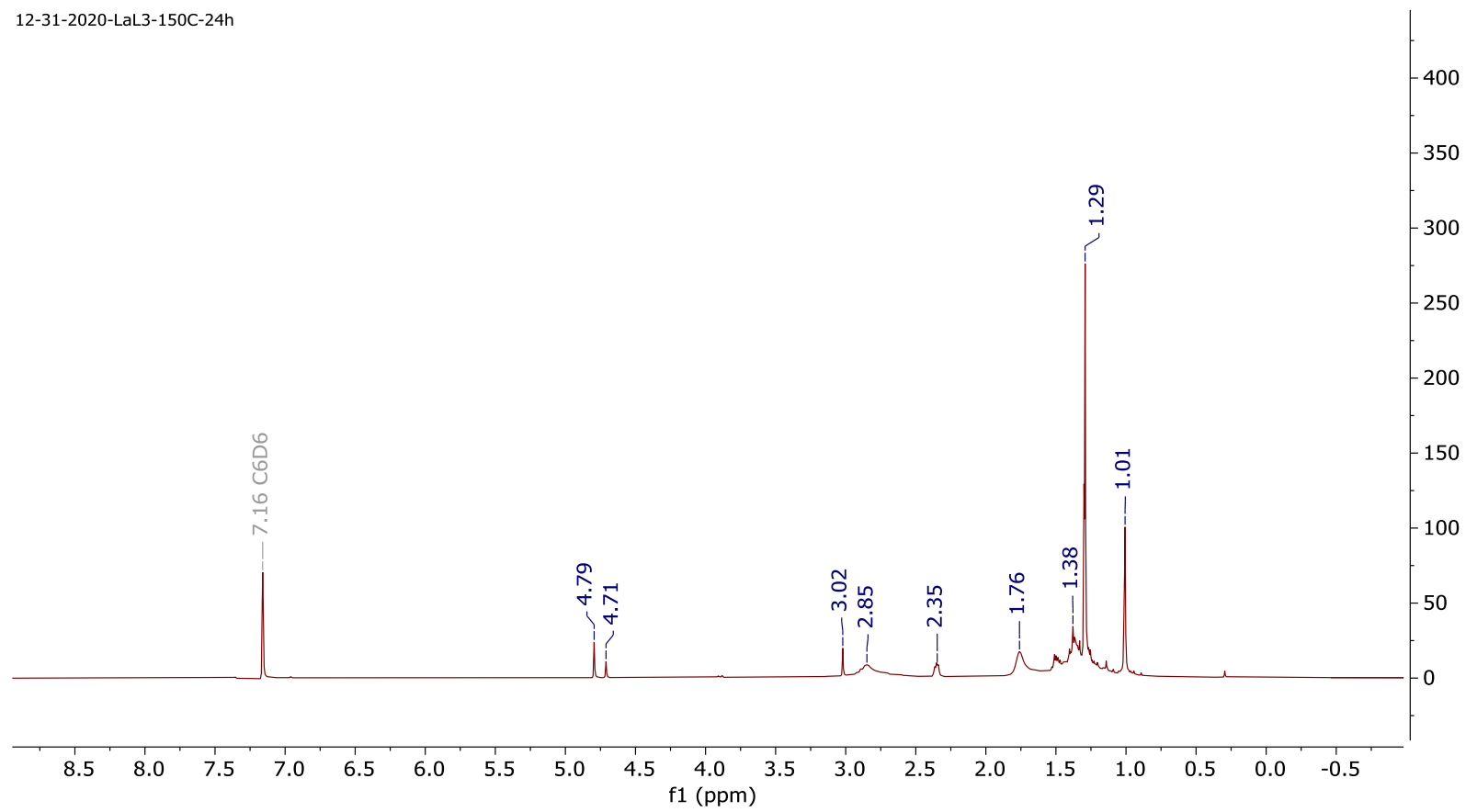


Figure S36. ${ }^{1} \mathrm{H}$ NMR spectra of 11 (a) pure sublimed sample and (b) after heating at $150{ }^{\circ} \mathrm{C}$ for 24 hours.

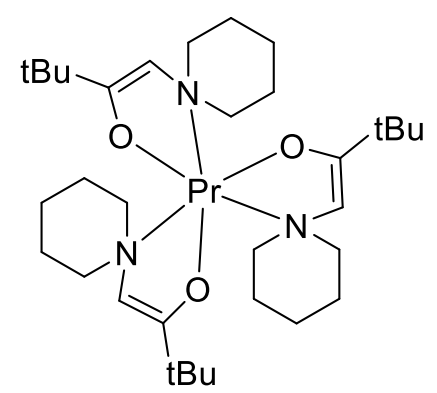

(a)

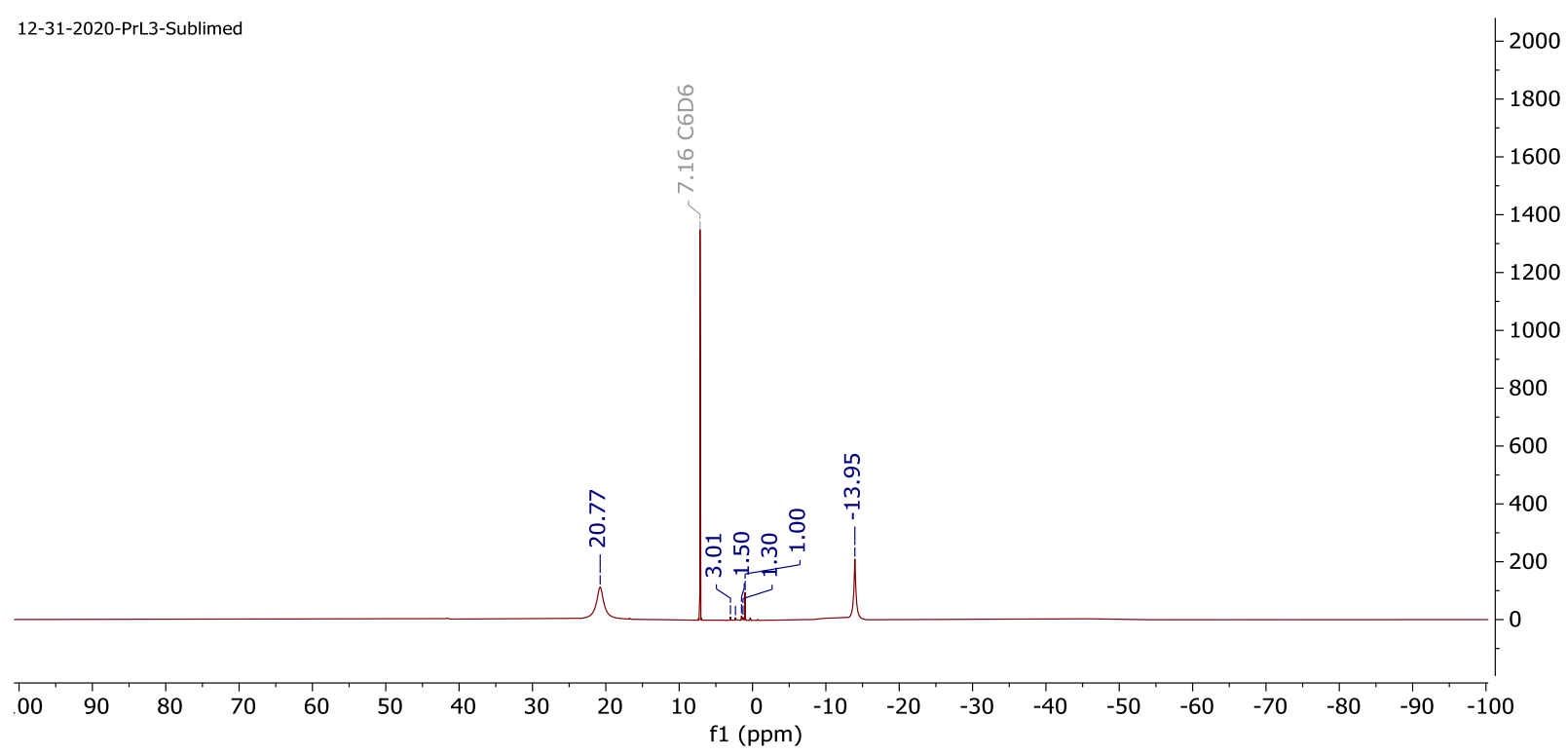

(b)

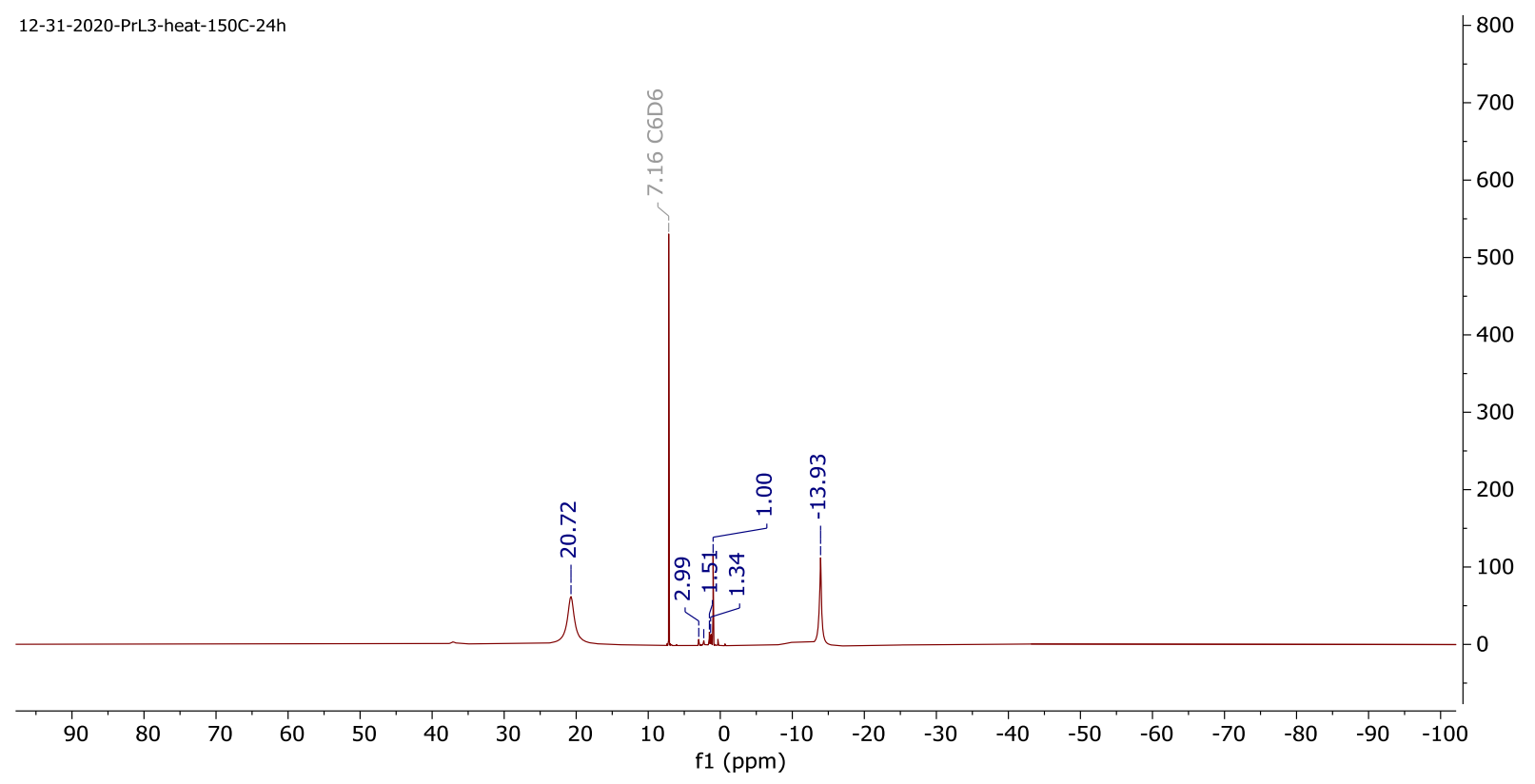


Figure S37. ${ }^{1} \mathrm{H}$ NMR spectra of 12 (a) pure sublimed sample and (b) after heating at $150{ }^{\circ} \mathrm{C}$ for 24 hours.<smiles>CC(C)CCN1C=C(CC(C)C)OP(N2CCCCC2)(N2CCCCC2)(N2CCCCC2)OC(CC(C)C)=CO1</smiles>

(a)

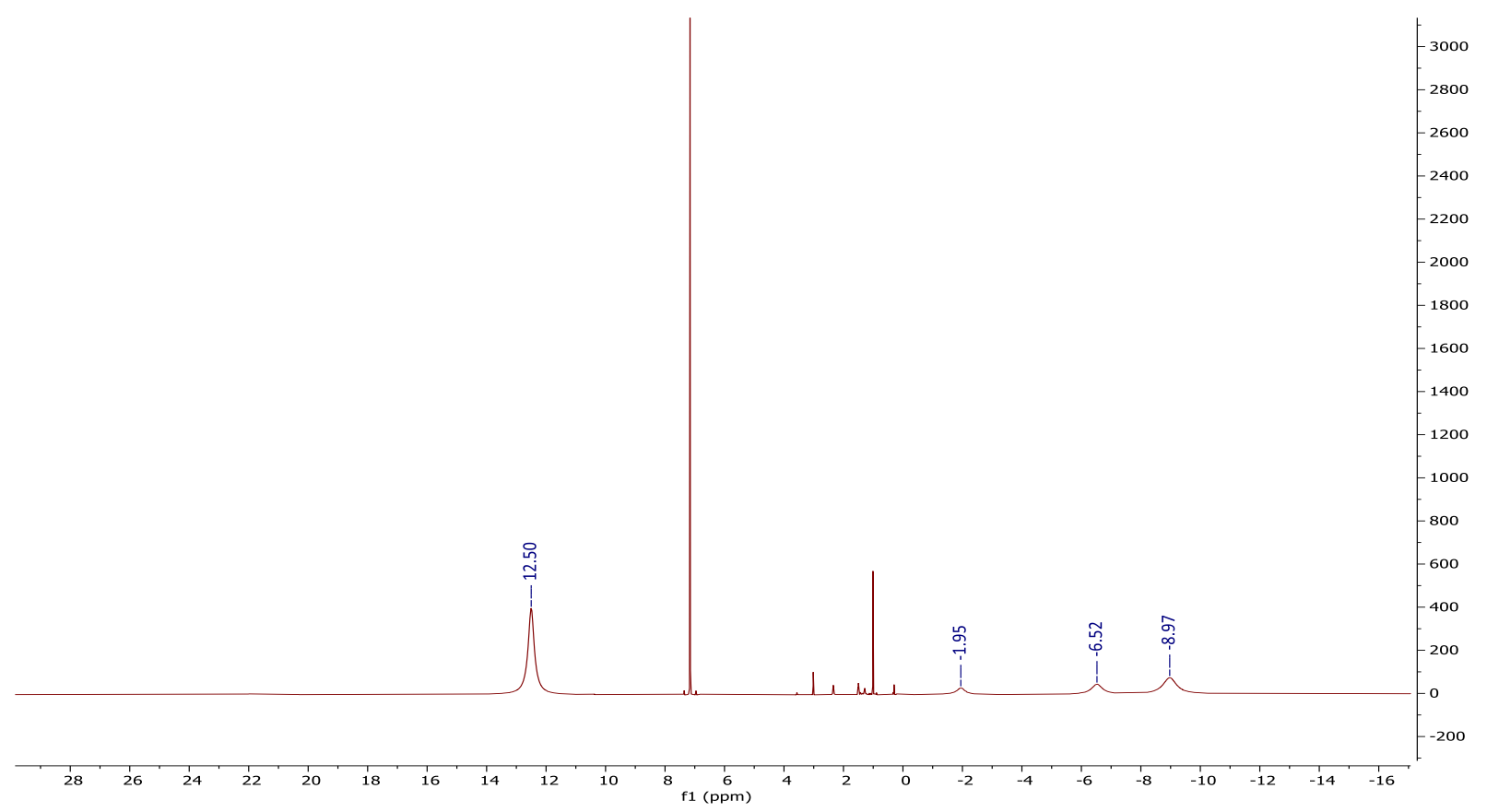


(b)

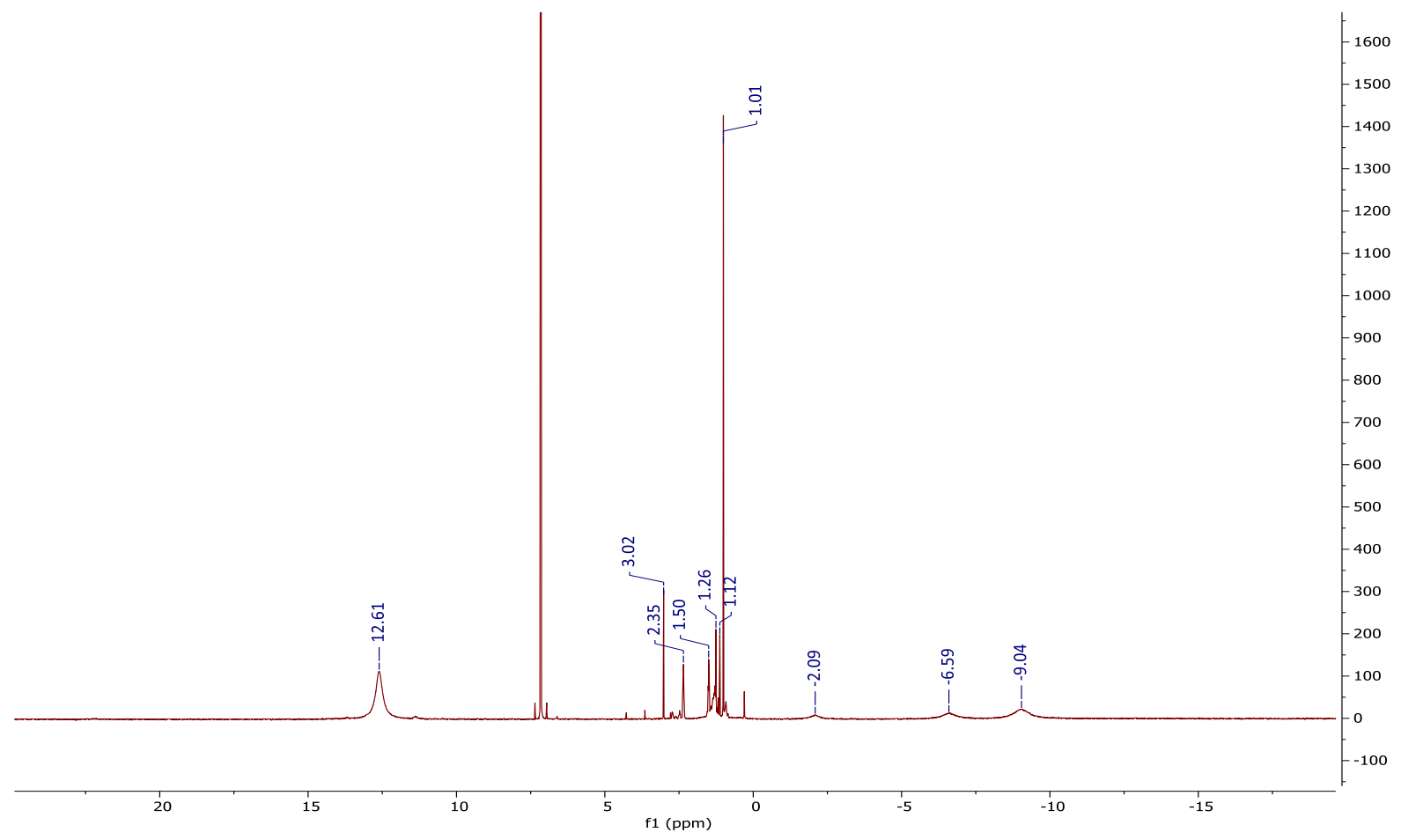


Figure S38. ${ }^{1} \mathrm{H}$ NMR spectra of 13 (a) pure sublimed sample and (b) after heating at $140{ }^{\circ} \mathrm{C}$ for 24 hours.

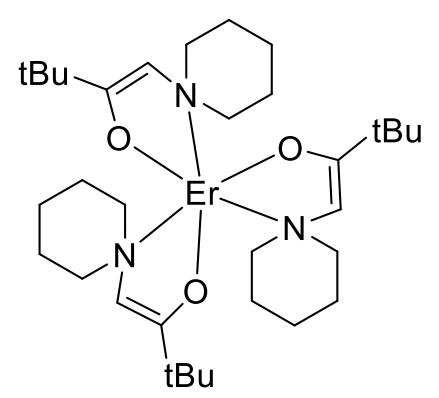

(a)

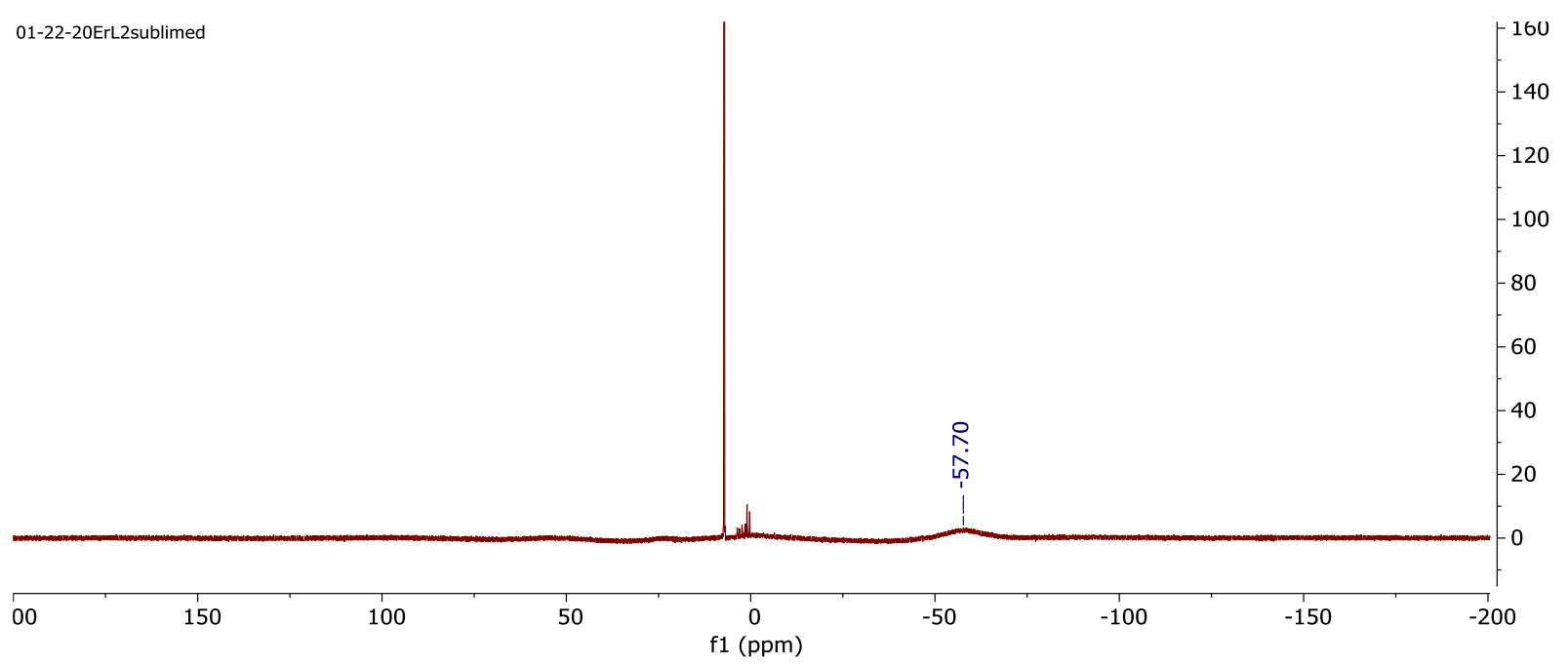

(b)

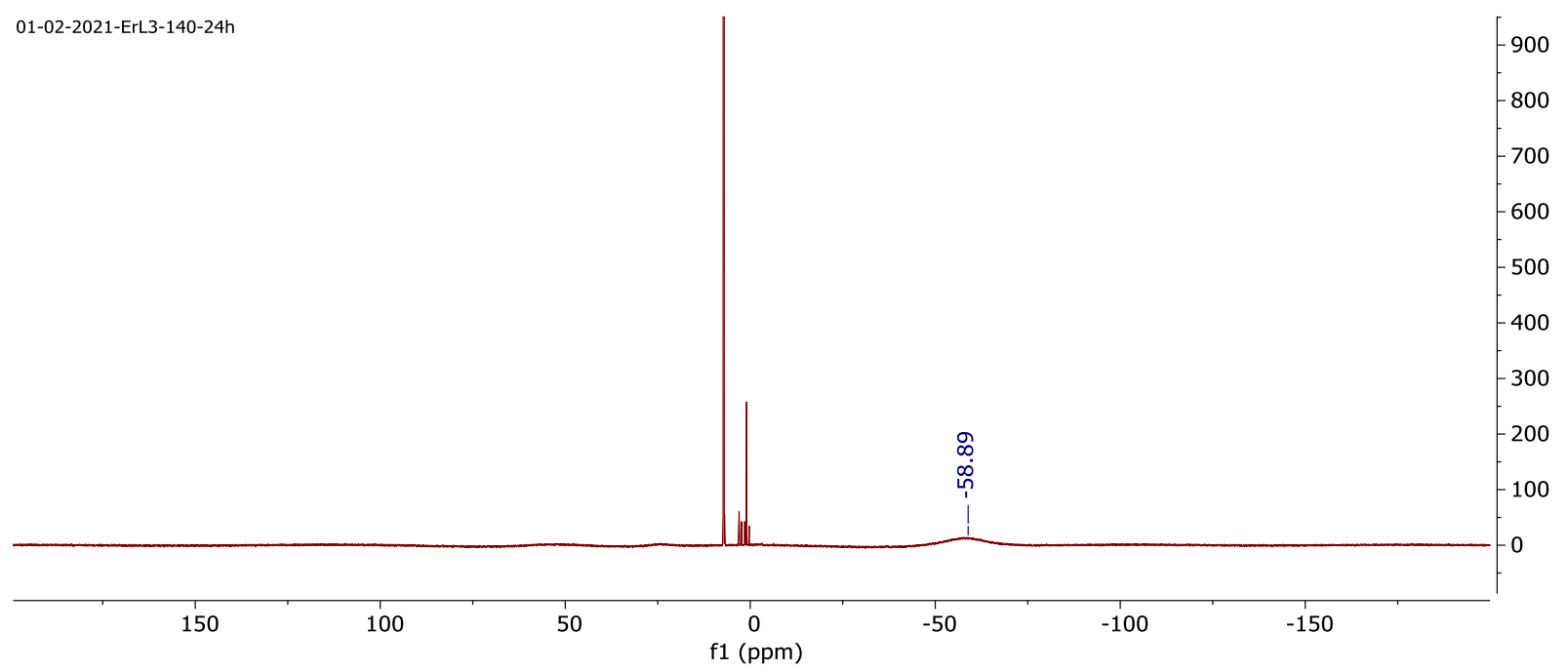


Figure S39. ${ }^{1} \mathrm{H}$ NMR spectra of 14 (a) pure sublimed sample and (b) after heating at $140{ }^{\circ} \mathrm{C}$ for 24 hours.<smiles>CC(C)CC1=CN(C2(N3CCCCC3)C=C(CC(C)C)OC2(OC(C)CC(C)C)N2CCCCC2)CCCC1</smiles>

(a)

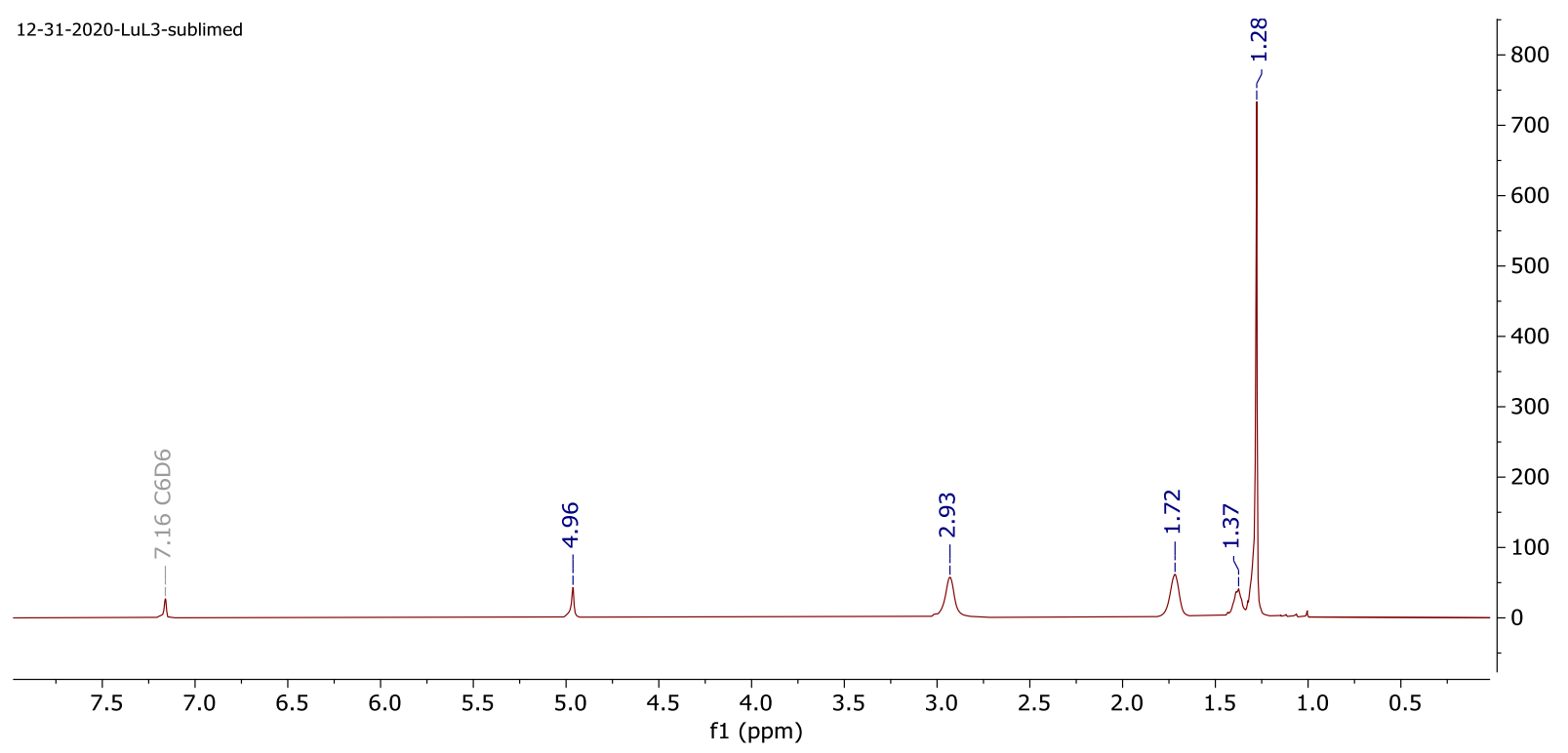


(b)

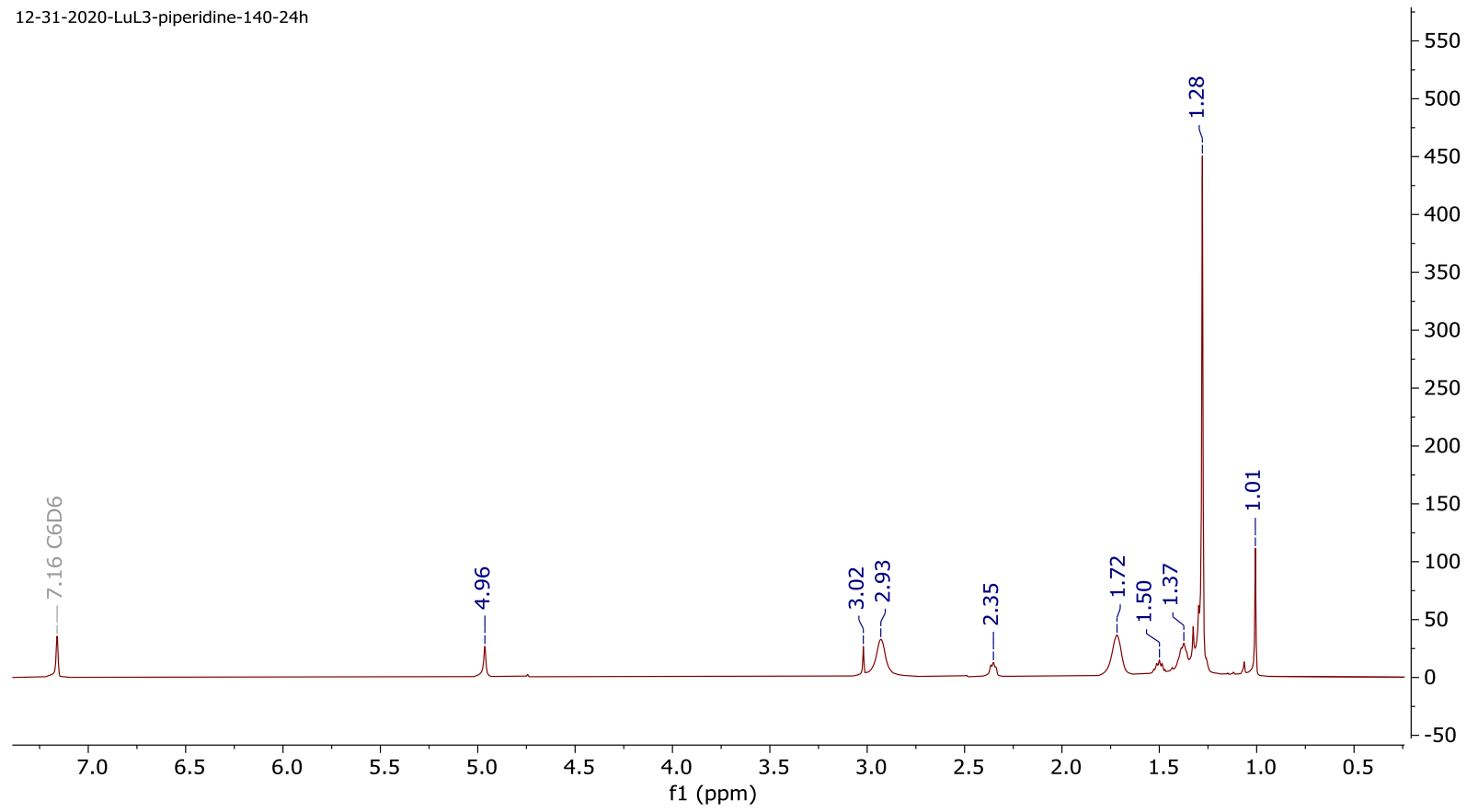


Figure S40. ${ }^{1} \mathrm{H}$ NMR spectra of 15 (a) pure sublimed sample and (b) after heating at $150{ }^{\circ} \mathrm{C}$ for 24 hours.

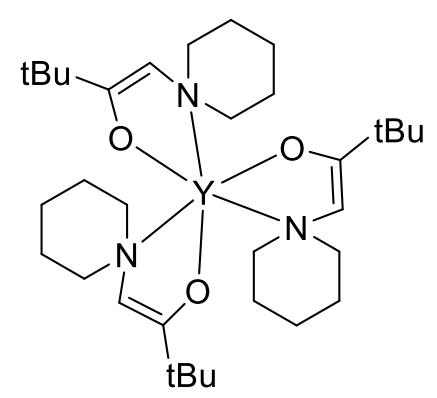

(a)

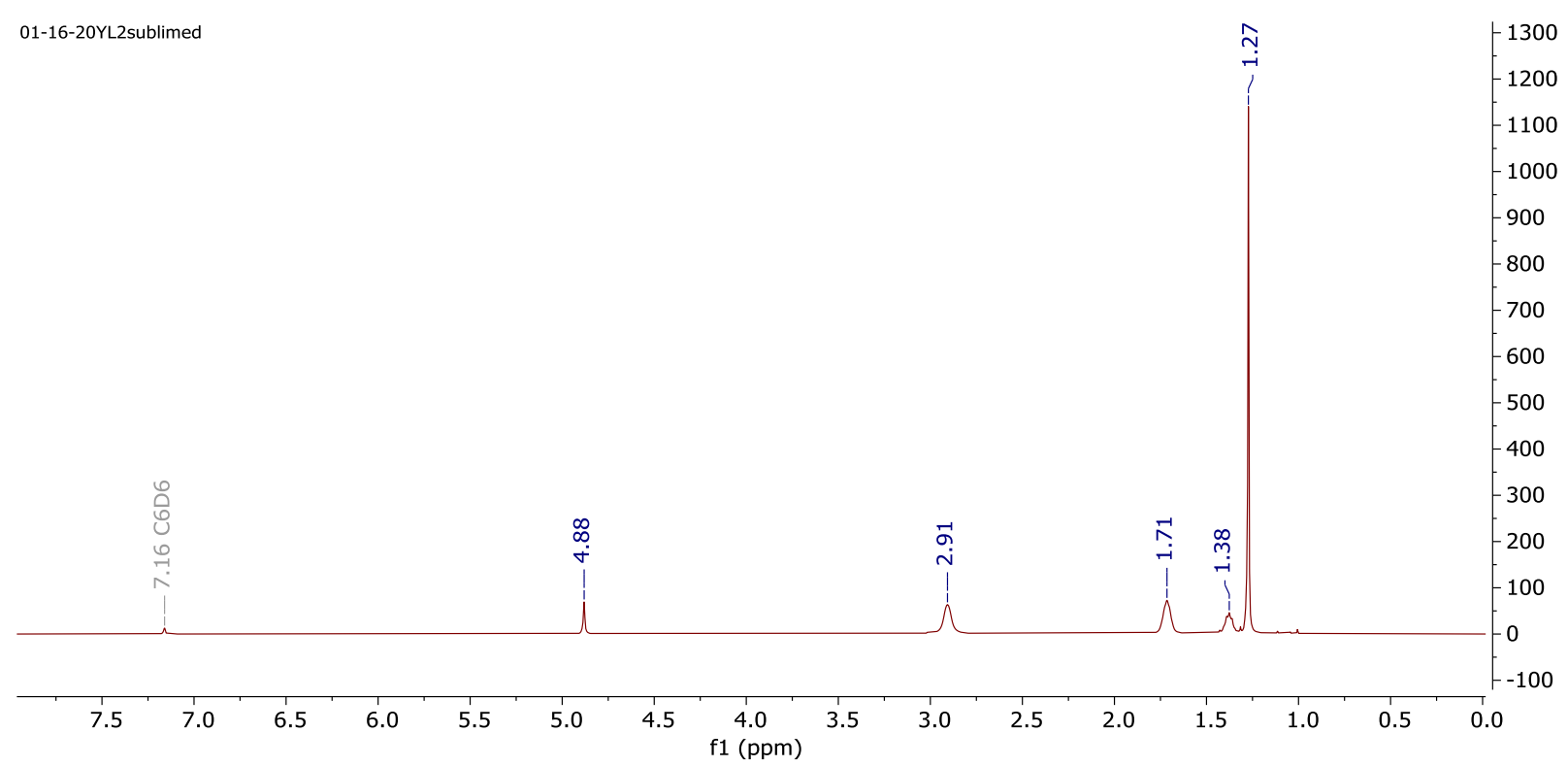


(b)

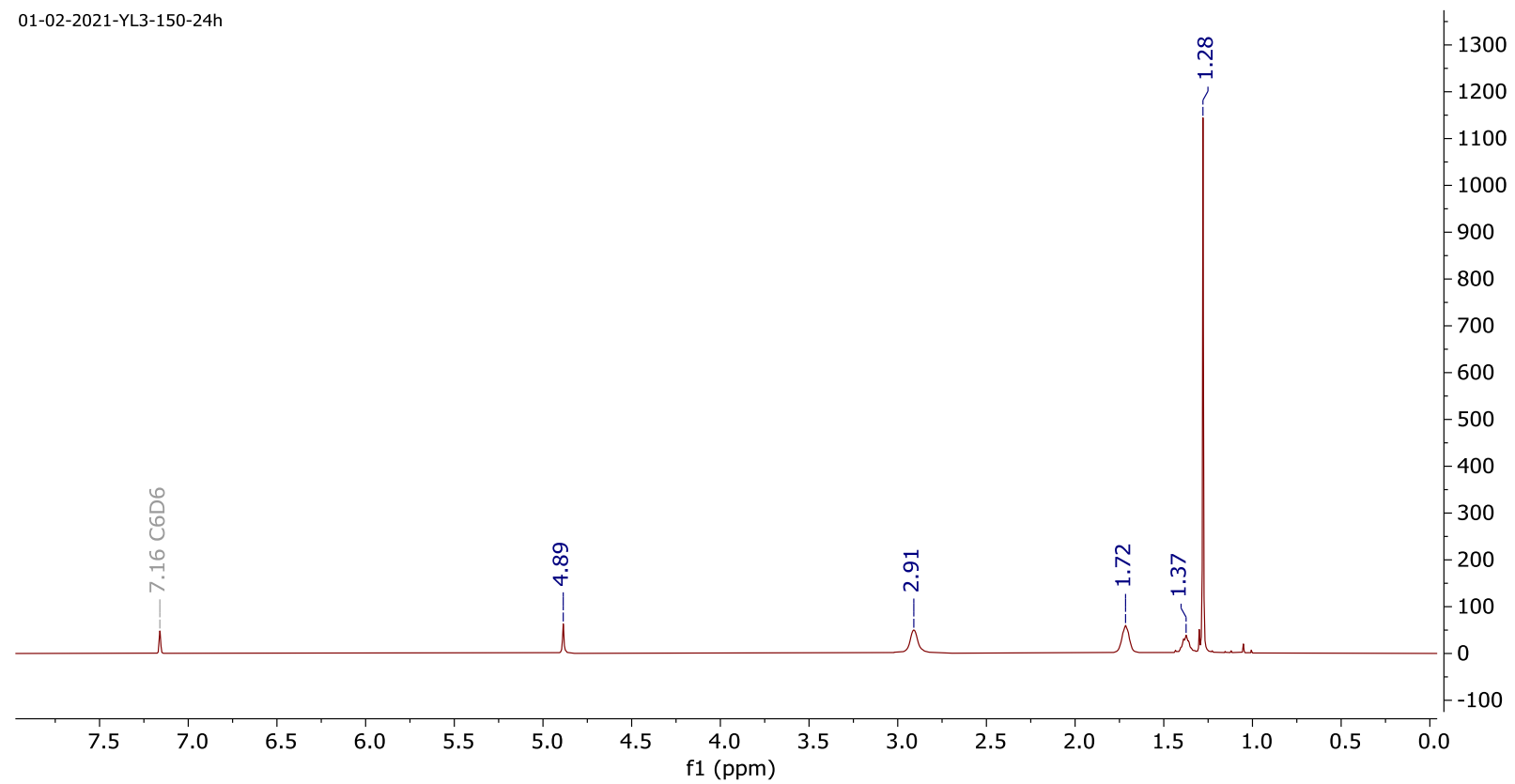


Figure S41. ${ }^{1} \mathrm{H}$ NMR spectrum of $\mathrm{L}^{3} \mathrm{H}$ to serve as a reference spectrum for Figures S35-40.<smiles></smiles>

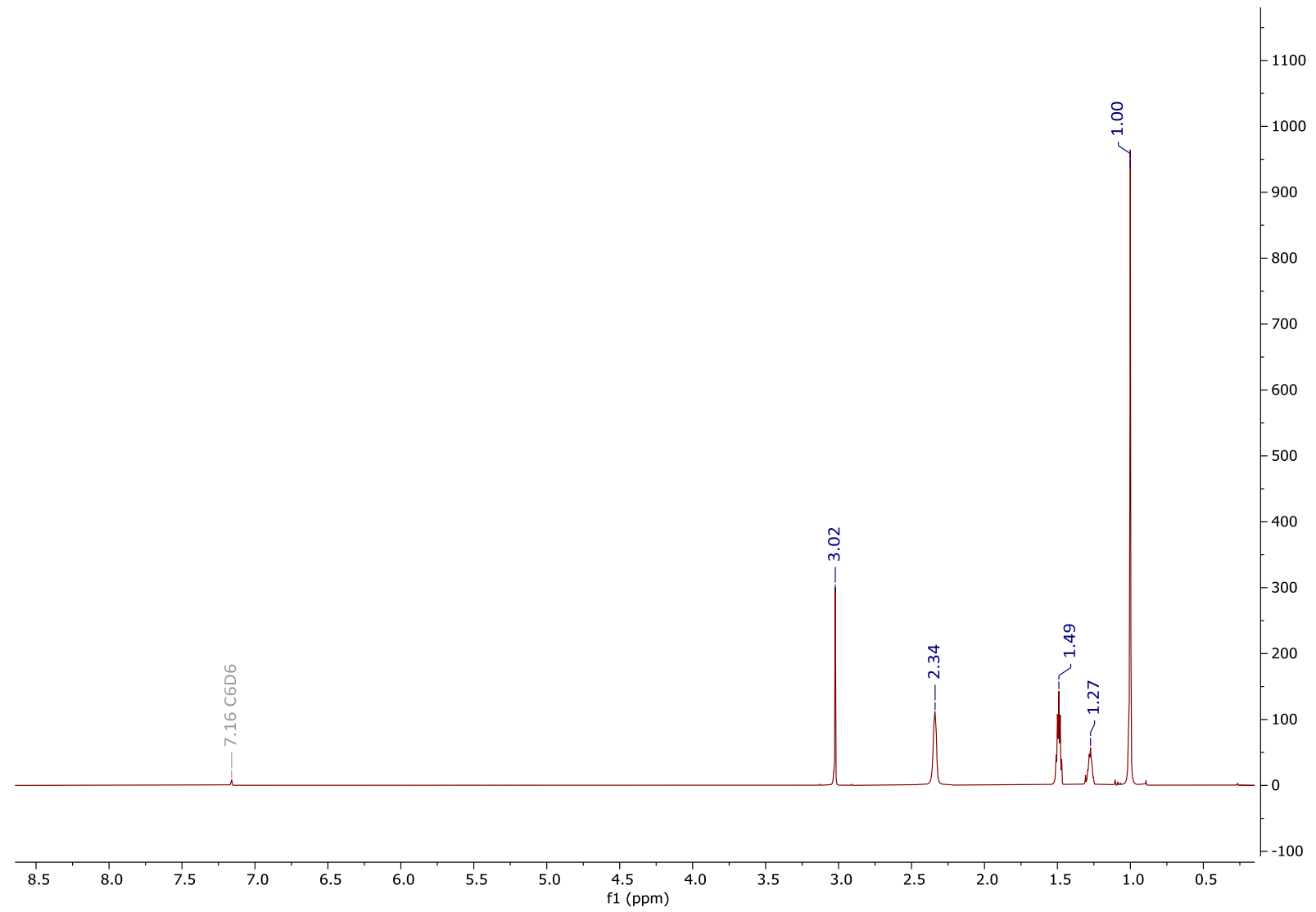

11-27-19-piperidine 
Figure S42. Perspective view of 9 with thermal ellipsoids at the $50 \%$ level.

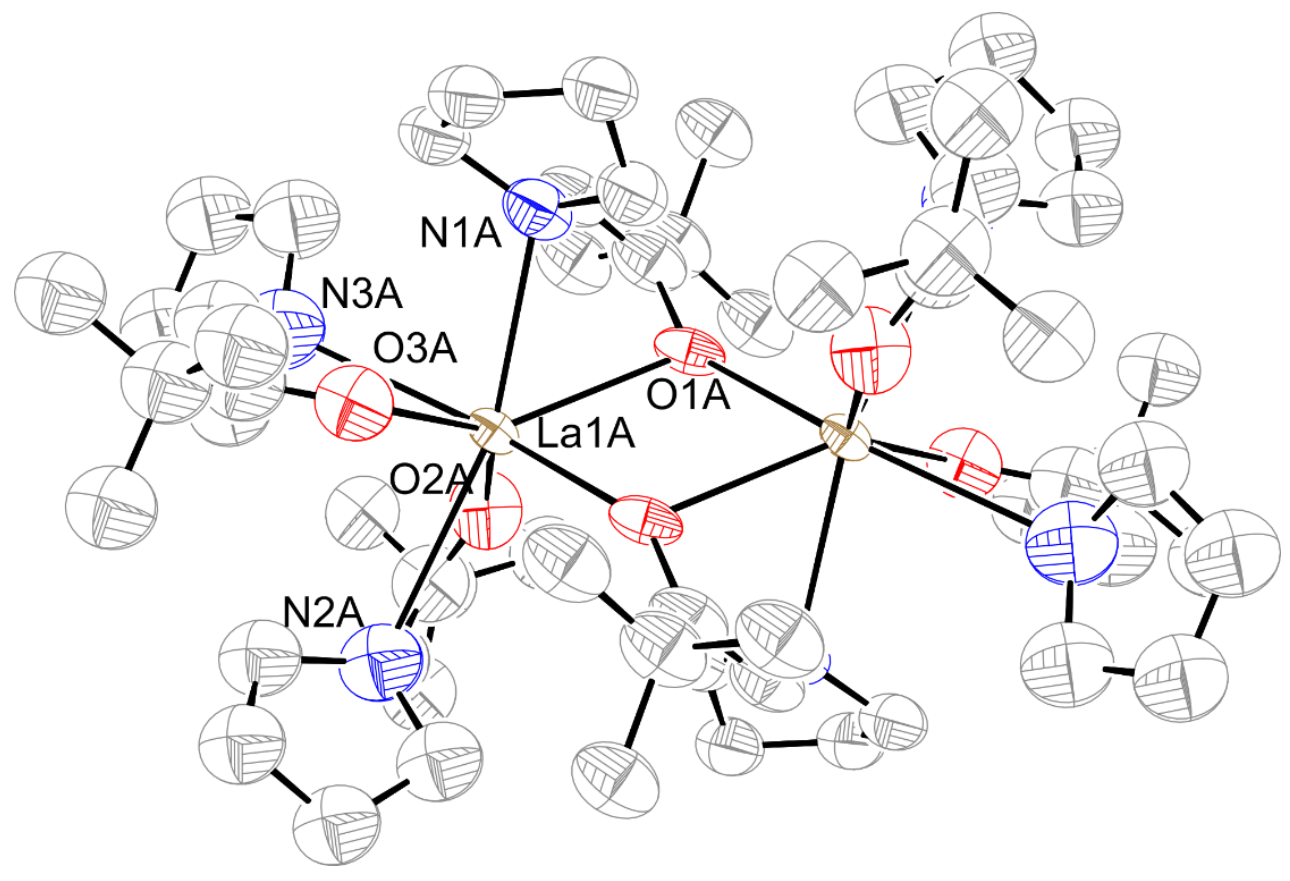

